University of Louisville

ThinkIR: The University of Louisville's Institutional Repository

Faculty Scholarship

$7-2013$

\title{
The impact of bars on disk breaks as probed by S4G imaging.
}

\author{
Juan Carlos Munoz-Mateos \\ National Radio Astronomy Observatory \\ Kartik Sheth \\ National Radio Astronomy Observatory \\ Armando Gil de Paz \\ Universidad Complutense de Madrid \\ Sharon Meidt \\ Max-Planck-Institut für Astronomie \\ E. Athanassoula \\ Aix Marseille Universite \\ See next page for additional authors
}

Follow this and additional works at: https://ir.library.louisville.edu/faculty

Part of the Astrophysics and Astronomy Commons

\section{Original Publication Information}

Munoz-Mateos, Juan Carlos, et al. "The Impact of Bars on Disk Breaks as Probed by S4G Imaging." 2013. The Astrophysical Journal 771(1): 30 pp.

This Article is brought to you for free and open access by ThinkIR: The University of Louisville's Institutional Repository. It has been accepted for inclusion in Faculty Scholarship by an authorized administrator of ThinkIR: The University of Louisville's Institutional Repository. For more information, please contact thinkir@louisville.edu. 


\section{Authors}

Juan Carlos Munoz-Mateos, Kartik Sheth, Armando Gil de Paz, Sharon Meidt, E. Athanassoula, Albert Bosma, Sebastien Comeron, Debra M. Elmegreen, Bruce G. Elmegreen, Santiago Erroz-Ferrer, Dimitri A. Gadotti, Joannah L. Hinz, Luis C. Ho, Benne W. Holwerda, Thomas H. Jarrett, Taehyun Kim, Johan H. Knapen, Jarkko Laine, Eija Laurikainen, Barry F. Madore, Karin Menendez-Delmestre, Trisha Mizusawa, Michael Regan, Heikki Salo, Eva Schinnerer, Mark Seibert, Ramin Skibba, and Dennis Zaritsky 


\title{
THE IMPACT OF BARS ON DISK BREAKS AS PROBED BY S ${ }^{4} \mathrm{G}$ IMAGING
}

\author{
Juan Carlos Muñoz-Mateos ${ }^{1}$, Kartik Sheth $^{1}$, Armando Gil de Paz ${ }^{2}$, Sharon Meidt ${ }^{3}$, E. Athanassoula ${ }^{4}$, \\ Albert Bosma ${ }^{4}$, Sébastien Comerón ${ }^{5}$, Debra M. Elmegreen ${ }^{6}$, Bruce G. Elmegreen ${ }^{7}$, Santiago Erroz-Ferrer $^{8,9}$, \\ Dimitri A. Gadotti ${ }^{10}$, Joannah L. Hinz ${ }^{11}$, Luis C. Ho ${ }^{12}$, Benne Holwerda ${ }^{13}$, Thomas H. Jarrett ${ }^{14}$, Taehyun Kim ${ }^{1,10,12,15}$, \\ Johan H. KNAPEN ${ }^{8,9}$, JarkKo Laine ${ }^{5}$, Eija Laurikainen ${ }^{5,16}$, Barry F. Madore ${ }^{12}$, Karin Menendez-Delmestre ${ }^{17}$, \\ Trisha Mizusawa ${ }^{1,18}$, Michael Regan ${ }^{19}$, HeikKi Salo ${ }^{5}$, Eva Schinnerer ${ }^{3}$, \\ MARK SEIBERT ${ }^{12}$, RAMIN SKIBba ${ }^{11}$, AND DenNis ZaritSKY ${ }^{11}$ \\ ${ }^{1}$ National Radio Astronomy Observatory/NAASC, 520 Edgemont Road, Charlottesville, VA 22903, USA; jmunoz@nrao.edu \\ ${ }^{2}$ Departamento de Astrofísica, Universidad Complutense de Madrid, E-28040 Madrid, Spain \\ ${ }^{3}$ Max-Planck-Institut für Astronomie, Königstuhl 17, D-69117 Heidelberg, Germany \\ ${ }^{4}$ Aix Marseille Universite, CNRS, LAM (Laboratoire d'Astrophysique de Marseille) UMR 7326, F-13388 Marseille, France \\ ${ }^{5}$ Department of Physical Sciences/Astronomy Division, University of Oulu, FIN-90014, Finland \\ ${ }^{6}$ Department of Physics and Astronomy, Vassar College, Poughkeepsie, NY 12604, USA \\ ${ }^{7}$ IBM Research Division, T.J. Watson Research Center, Yorktown Hts., NY 10598, USA \\ ${ }^{8}$ Instituto de Astrofísica de Canarias, E-38205 La Laguna, Spain \\ ${ }^{9}$ Departamento de Astrofísica, Universidad de La Laguna, E-38206 La Laguna, Spain \\ ${ }^{10}$ European Southern Observatory, Casilla 19001, Santiago 19, Chile \\ ${ }^{11}$ University of Arizona, 933 N. Cherry Ave, Tucson, AZ 85721, USA \\ 12 The Observatories, Carnegie Institution of Washington, 813 Santa Barbara Street, Pasadena, CA 91101, USA \\ ${ }^{13}$ European Space Agency, ESTEC, Keplerlaan 1, 2200-AG, Noordwijk, The Netherlands \\ ${ }^{14}$ Astrophysics, Cosmology and Gravity Centre, Department of Astronomy, University of Cape Town, Private Bag X3, Rondebosch 7701, South Africa \\ ${ }^{15}$ Astronomy Program, Department of Physics and Astronomy, Seoul National University, Seoul 151-742, Korea \\ ${ }^{16}$ Finnish Centre for Astronomy with ESO (FINCA), University of Turku, Finland \\ ${ }^{17}$ Observatorio do Valongo, Universidade Federal de Rio de Janeiro, Ladeira Pedro Antöonio, 43, Saude CEP 20080-090, Rio de Janeiro, RJ, Brazil \\ ${ }^{18}$ Florida Institute of Technology, Melbourne, FL 32901, USA \\ ${ }^{19}$ Space Telescope Science Institute, 3700 San Martin Drive, Baltimore, MD 21218, USA \\ Received 2012 August 16; accepted 2013 April 18; published 2013 June 17
}

\begin{abstract}
We have analyzed the radial distribution of old stars in a sample of 218 nearby face-on disks, using deep $3.6 \mu \mathrm{m}$ images from the Spitzer Survey of Stellar Structure in Galaxies. In particular, we have studied the structural properties of those disks with a broken or down-bending profile. We find that, on average, disks with a genuine single-exponential profile have a scale length and a central surface brightness which are intermediate to those of the inner and outer components of a down-bending disk with the same total stellar mass. In the particular case of barred galaxies, the ratio between the break and the bar radii $\left(R_{\mathrm{br}} / R_{\mathrm{bar}}\right)$ depends strongly on the total stellar mass of the galaxy. For galaxies more massive than $10^{10} M_{\odot}$, the distribution is bimodal, peaking at $R_{\mathrm{br}} / R_{\mathrm{bar}} \sim 2$ and $\sim 3.5$. The first peak, which is the most populated one, is linked to the outer Lindblad resonance of the bar, whereas the second one is consistent with a dynamical coupling between the bar and the spiral pattern. For galaxies below $10^{10} M_{\odot}$, breaks are found up to $\sim 10 R_{\text {bar }}$, but we show that they could still be caused by resonances given the rising nature of rotation curves in these low-mass disks. While not ruling out star formation thresholds, our results imply that radial stellar migration induced by non-axisymmetric features can be responsible not only for those breaks at $\sim 2 R_{\text {bar }}$, but also for many of those found at larger radii.
\end{abstract}

Key words: galaxies: evolution - galaxies: photometry - galaxies: spiral - galaxies: stellar content - galaxies: structure

Online-only material: color figures, figure set, machine-readable tables

\section{INTRODUCTION}

The initial conditions under which galaxies form and the physical mechanisms that govern their subsequent evolution are encoded in the stellar structure of galaxy disks. Hence, the radial profile of the stellar disk is a powerful tool for probing galaxy evolution over cosmic time.

Light profiles of galactic disks have generally been described by an exponential law (Freeman 1970) with a truncation or a break ${ }^{20}$ at the outer edge of the disk (van der Kruit 1979).

20 The terms "break" and "truncation" are often used somewhat interchangeably in the literature. Here, we prefer to use "break" when talking about changes in slope in the main disk of galaxies (the subject of this paper) and leave "truncation" for the features seen much farther out in edge-on disks (see Martín-Navarro et al. 2012).
Subsequent deeper observations showed that the sharp cutoffs found by van der Kruit are better described by a doubleexponential profile (Pohlen et al. 2002), where the slope of the outer exponential is steeper than that of the inner one; these are known as down-bending profiles. In contrast to these, some disks have been observed with an outer disk which has a shallower light profile than the inner exponential (Erwin et al. 2005; Pohlen $\&$ Trujillo 2006); these are referred to as up-bending profiles.

The latest data show that systems with a single-exponential profile are the exception rather than the rule in the local universe (Erwin et al. 2005; Pohlen \& Trujillo 2006). By analyzing optical light profiles of $\sim 90$ nearby late-type spirals (Sb-Sdm), Pohlen \& Trujillo (2006) showed that only $10 \%$ exhibited singleexponential profiles (Type I). Roughly $60 \%$ were found to have down-bending profiles (Type II) and the remaining 30\% 
showed an up-bending profile (Type III). Erwin et al. (2008; E08 hereafter) performed a similar study on a sample of 66 nearby barred, early-type disks ( $\mathrm{S} 0-\mathrm{Sb}$ ), and concluded that $27 \%, 42 \%$, and $24 \%$ of their galaxies exhibited Type I, II, and III profiles, respectively; the remaining $6 \%-7 \%$ showed a combination of Type II and III profiles. Double-exponential profiles are also common in very late-type systems. Within a sample of 136 Im, Sm and blue compact dwarf galaxies, Hunter \& Elmegreen (2006) showed that 50 of them presented down-bending profiles, whereas 12 exhibited up-bending ones. The ubiquity of multisloped profiles suggests that they either form easily (perhaps through more than one mechanism) and/or are very long-lived; otherwise only a small fraction of galaxies would exhibit these features.

The down-bending profiles exhibit a characteristic U-shaped color profile, both locally (Bakos et al. 2008) and at high redshift (Azzollini et al. 2008a). The color gets bluer out to the break radius, as one would expect from an inside-out formation scenario, and then becomes redder past the break radius. Numerical simulations by Roškar et al. (2008) and Sánchez-Blázquez et al. (2009) have attributed this excess of red emission in galactic outskirts to radial stellar migration. According to these models, more than half of the old stars currently found outside the break radius were actually born inside it, and later migrated outward. This scenario seems to be borne out by observations of resolved stellar populations across the break radius in lower-mass galaxies (de Jong et al. 2007; Radburn-Smith et al. 2011) and two-dimensional (2D) optical spectra (Yoachim et al. 2010, 2012).

Disk breaks have been detected up to $z \sim 1$ (Pérez 2004), and studies have suggested that the break radius increases with time (Trujillo \& Pohlen 2005; Azzollini et al. 2008b). In principle, this suggests that these features could be intimately linked to the inside-out growth of galactic disks. However, the picture could well be different in barred galaxies. Indeed simulations show that bars are expected to grow with time (see Athanassoula 2003 and references therein), so the temporal growth of the break radius could be driven by that of the bar, even in the absence of a significant inside-out evolution of the disk itself.

One of the first explanations for the physical origin of disk breaks appealed to the conservation of angular momentum of the infalling material. Van der Kruit (1987) showed that a collapsing gaseous sphere settles onto a disk with a break corresponding to the maximum specific angular momentum of the original spherical cloud. However, in a more realistic scenario where gas is deposited in the outer parts with varying angular momenta and timescales, the notion of a constant cutoff radius becomes less likely.

Moreover, angular momentum can be subsequently redistributed if non-axisymmetric features such as bars form, as shown by $N$-body simulations (Sellwood 1980; Athanassoula 2003). Bars tend to drive material within the corotation resonance $(\mathrm{CR})$ to smaller radii, and material outside CR outward, thus increasing the central stellar density, while flattening the disk. Interestingly, though, bars themselves can also give rise to radial breaks, as described by Debattista et al. (2006). These simulations are quite demanding in terms of the number of particles required to properly sample the outer disk, and this comes at the expense of using a rigid halo rather than a live one. In this regard, Foyle et al. (2008) extended the study of Debattista et al. (2006) by using live halos and exploring a wider range of galaxy properties. They found that the onset of breaks seems to be governed by the ratio between the halo spin parameter $\lambda$ and the disk mass fraction $m_{\mathrm{d}}$. It has been also shown that a live halo can absorb a substantial amount of angular momentum from the bar (Athanassoula 2002) — in fact much more than the tenuous outer parts of the disk-which will in turn affect the bar properties, as well as the angular momentum absorbed by the outer disk. A live halo might therefore modify the results of simulations quantitatively, but perhaps not qualitatively.

If the break is linked to the bar then it will be inside its outer Lindblad resonance (OLR). However, if the bar drives a spiral by nonlinear mode coupling (Tagger et al. 1987; Sygnet et al. 1988), then the break should form inside the OLR of the spiral (Debattista et al. 2006), which is located farther out than the bar OLR, as the spiral pattern speed is lower than that of the bar. Interestingly, recent simulations also show that under a bar-spiral coupling, breaks can also form at the spiral CR (Minchev et al. 2012). Finally, a break can come from manifolddriven spirals, in which case its radius is not linked to the outer resonance of the spiral, but is nevertheless located not far from it (Athanassoula 2012).

From an observational point of view, E08 found that the break radius in many down-bending profiles is located at two or three times the bar radius, and thus proposed a possible connection with the OLR. This is based on the fact that outer rings, which have been traditionally associated with the OLR, have a radius around twice the bar radius (Kormendy 1979; Athanassoula et al. 1982; Buta \& Crocker 1993). The real picture, however, can be substantially more complicated since the precise location of resonances depends on the pattern speed of the bar (and spiral arms if sufficiently massive) and the rotation curve of the galaxy, both of which may change over time.

In contrast to the angular momentum framework, some studies favor star formation thresholds as a likely culprit for disk breaks. If there is a critical gas threshold for star formation (Kennicutt 1989), then this may give rise to a break in the radial profiles. However, the discovery of extended UV emission well beyond the main optical disk of many galaxies (Gil de Paz et al. 2005; Thilker et al. 2005; Zaritsky \& Christlein 2007) challenges this view. According to the survey by Thilker et al. (2007), in roughly $20 \%$ of nearby galaxies this extended emission is in the form of structured star-forming knots at extreme radii; other disks (around 10\%) present abnormally large and uniform areas with very blue UV-NIR colors, although not so far from the main disk. A follow-up study by Lemonias et al. (2011), using a larger sample and a slightly different classification scheme, found the incidence of XUV disks to be below but close to $20 \%$.

The existence of star formation activity at such extreme radii in some galaxies also implies that, at least in these cases, the break radius might not necessarily correspond to the material with the maximum angular momentum. As shown by Christlein \& Zaritsky (2008), the rotation curve probed by star-forming knots up to twice the optical radius seems to be flat, meaning that this material has large angular momentum. Star formation in the outskirts of disks could be triggered by turbulent compression; together with other mechanisms driving star formation in the inner parts, this might actually yield down-bending profiles (Elmegreen \& Hunter 2006). Thus, depending on whether the galaxy has an extended gas disk or not, and where the transition between the inner and outer gas profiles takes place, the superposition of these different mechanisms can lead to either down- or up-bending profiles. In this context, XUVdisk host galaxies are likely the ones with the shallowest outer gas profiles. Even in these extreme cases a secondary outer truncation might be present due to a star formation threshold 
associated with either a sharp decrease in the gas density or decrease in the efficiency of star formation mechanism at larger radii.

While resonances and/or star formation thresholds are normally invoked to reproduce down-bending profiles, up-bending ones are often explained through external mechanisms. Younger et al. (2007) showed that minor mergers can yield up-bending profiles: gas inflows toward the center of the galaxy would steepen the inner profile, while the outer one would expand as angular momentum is transferred outward during the interaction. Apart from minor mergers, Minchev et al. (2012) demonstrated that smooth gas accretion can also lead to a flat outer disk, creating not only pure up-bending profiles (Type III) but also composite ones (Type II + III).

Going back to down-bending profiles, among the different mechanisms that can produce and/or modify this kind of breaks, bars should receive special attention. Their non-axisymmetric potentials induce radial transfer of gas, stars, and angular momentum, which can lead to a substantial rearrangement of the disk structure. Parameters like the break radius or the ratio between the inner and outer disk scale lengths are therefore expected to be different in barred and unbarred galaxies, and perhaps correlated with properties of the bar such as its length or ellipticity.

There is consensus that the bar fraction in the local universe is around $\sim 65 \%$ in the infrared (Eskridge et al. 2000; Knapen et al. 2000; Whyte et al. 2002; Menéndez-Delmestre et al. 2007; Marinova \& Jogee 2007). Trends of the bar fraction with mass, color, morphological type, and environment have been also reported in the literature (see, e.g., Nair \& Abraham 2010; Masters et al. 2011; Skibba et al. 2012, and references therein). Moreover, it has been shown that the redshift evolution of the bar fraction is strongly dependent on the total stellar mass of galaxies (Sheth et al. 2008). The bar fraction for massive disks is roughly constant up to $z \sim 0.8$, but it declines considerably with increasing redshift for low-mass galaxies. Simulations show that the bar instability grows faster if the disk is dynamically cold and/or rotationally supported (Athanassoula \& Sellwood 1986; Sheth et al. 2012), and therefore this differential evolution of the bar fraction has important implications for the assembly of galactic disks. In particular, if bars are responsible for disk breaks, and given that low-mass disks seem to have acquired their bars only recently, it is worth investigating whether this translates into systematic variations of the break properties (radius, scale-length ratio, etc.) as a function of stellar mass.

Previous studies on disk breaks at low and high redshifts have relied on rest-frame optical images. Probing galactic structure through optical light profiles is hampered by the radial variations in dust content, metallicity, and mass-to-light ratio, all of which can make the derived properties of disks significantly different from those of the underlying old stellar population. In order to overcome these limitations, here we rely on the Spitzer Survey of Stellar Structure in Galaxies ( $S^{4} G$; Sheth et al. 2010), a census of more than 2300 galaxies within $40 \mathrm{Mpc}$ imaged at $3.6 \mu \mathrm{m}$ and $4.5 \mu \mathrm{m}$. The $\mathrm{S}^{4} \mathrm{G}$ data set probes stellar surface densities as low as $1 M_{\odot} \mathrm{pc}^{-2}$, and therefore constitutes an ideal benchmark to study the outskirts of galactic disks. Given the large number of galaxies included in the survey, subsets of several hundreds objects can be easily assembled after slicing the parent sample according to different selection criteria, thus providing unprecedented statistical power at these wavelengths. Here, we present a first analysis of disk breaks for a sample of more than 200 face-on disks, with stellar masses greater than $\sim 2 \times 10^{9} M_{\odot}$.

The paper is organized as follows. In Section 2, we describe the selection criteria used to assemble our sample from the parent $S^{4} G$ survey. Section 3 deals with the technical aspects of the analysis; in particular, we detail the data processing and profile measurement (Section 3.1), the classification of disk profiles (Section 3.2), and the measurement of the properties of disks (Section 3.3), and bars (Section 3.4). The main scientific results are discussed in Section 4, and we summarize our main conclusions in Section 5.

\section{SAMPLE SELECTION}

The full $\mathrm{S}^{4} \mathrm{G}$ sample comprises of a total of more than 2300 nearby galaxies. They were selected from the HyperLEDA database (Paturel et al. 2003) according to the following selection criteria: radio-derived radial velocity $v_{\text {radio }}<3000 \mathrm{~km} \mathrm{~s}^{-1}$ (which corresponds to $d \lesssim 40 \mathrm{Mpc}$ for $H_{0}=71 \mathrm{~km} \mathrm{~s}^{-1} \mathrm{Mpc}^{-1}$ ), Galactic latitude $|b|>30^{\circ}$, total corrected blue magnitude $m_{B_{\text {corr }}}<15.5$ and blue light isophotal diameter $D_{25}>1$ '. 0 .

For the present work, we started with the 827 galaxies that were processed first through the $S^{4} \mathrm{G}$ pipelines (mosaic construction, object masking, and ellipse profile fitting; see Section 3.1). On this subset of galaxies, we applied three selection cuts based on morphology, stellar mass, and inclination. We selected only disk-like galaxies ranging from S0's to Sd's (that is, having numerical types $-3 \leqslant T \leqslant 7$ ), using the optical morphological types compiled in HyperLEDA. Sdm and Sm galaxies, while still exhibiting a spiral disk, usually present a patchy and asymmetric morphology that complicates the measurement and interpretation of radial profiles, so they were left out from our sample.

We also decided to restrict our analysis to a well-defined range in stellar mass. Global properties of galaxies, such as color, star formation rate, stellar age, metallicity, gas fraction, etc., vary with both the total stellar mass and the morphological type (Boselli et al. 1997; Kauffmann et al. 2003; Brinchmann et al. 2004; Tremonti et al. 2004; Schiminovich et al. 2007). However, the trends seem to be tighter and better defined when plotted against stellar mass, hinting that this is the main parameter that, at least to first order, governs most of a galaxy's evolutionary path. Also, basing our analysis on the stellar mass-or a reasonable luminosity proxy-makes it easier to compare our results with those at higher redshifts, where morphological studies are challenging, because of both coarser resolution and intrinsic departures from the classical Hubble types.

To that aim, here we use the $3.6 \mu \mathrm{m}$ luminosity as a stellar mass tracer. It is worth noting that sources other than old stars might contaminate the emission at this wavelength. Meidt et al. (2012) were able to isolate the old stellar light in a test sample of six $\mathrm{S}^{4} \mathrm{G}$ galaxies, by applying an independent component analysis to the corresponding 3.6 and $4.5 \mu \mathrm{m}$ images. They concluded that hot dust and polycyclic aromatic hydrocarbons together may account for $5 \%-15 \%$ of the total integrated light at $3.6 \mu \mathrm{m}$ (see also Zibetti \& Groves 2011), while intermediateage stars with low mass-to-light ratios do not contribute more than $5 \%$. These values are low enough so as not to compromise the usefulness of the $3.6 \mu \mathrm{m}$ luminosity as a stellar mass proxy.

Absolute magnitudes at $3.6 \mu \mathrm{m}$ were computed from the asymptotic apparent magnitudes obtained with our pipeline (see Section 3.1). Whenever possible, we relied on the mean redshiftindependent distances provided by the NASA Extragalactic 
Database (NED). In the absence of these values, we estimated the distance to each galaxy from its radial velocity $v_{\text {radio }}$ and our adopted $H_{0}$. We retained only galaxies with $M_{3.6 \mu \mathrm{m}}(\mathrm{AB})<$ $-18 \mathrm{mag}$. Using the stellar mass-to-light ratio at $3.6 \mu \mathrm{m}$ derived by Eskew et al. (2012; see Appendix A), our magnitude cut selects galaxies with stellar masses larger than $\sim 2 \times 10^{9} M_{\odot}$.

Finally, we required our galaxies to be at least moderately face-on, having an axial ratio $b / a>0.5\left(i<60^{\circ}\right)$ at $\mu_{3.6 \mu \mathrm{m}}=$ 25.5 AB mag $\operatorname{arcsec}^{-2}$, which we take as our fiducial surface brightness level for the outer parts of disks. In more inclined galaxies, the shape of the isophotes can be strongly distorted by the vertical structure of the disk, bulge, and stellar halo, thus precluding a straightforward interpretation of the light profiles. Moreover, in order to investigate the role of bars in shaping disk breaks, we need to obtain the deprojected values of properties such as bar length or its ellipticity; by focusing on galaxies close to face-on we minimize the impact of errors in the assumed inclination angle.

After applying these three selection criteria we are left with 248 galaxies. After a visual inspection, we removed 30 galaxies that were not suitable for our analysis. These mostly include highly inclined early-type disks such as the Sombrero galaxy, whose low axial ratios are due to the spheroidal component. We also removed some highly disturbed galaxies like NGC 0275, as well as galaxies whose radial profiles are unreliable due to very bright foreground stars in the field of view (such as NGC 6340).

Our final sample thus contains 218 galaxies, including both barred and unbarred ones, whose main properties are quoted in Table 1. Figure 1 shows the distribution of morphological types and absolute $3.6 \mu \mathrm{m}$ magnitudes in both the parent sample of 827 processed galaxies and the final sample. The images and radial profiles of these galaxies, together with quantitative measurements of their structural properties, are presented in Appendix B.

\section{ANALYSIS}

\subsection{Data Processing and Profile Measurement}

A description of the different parts comprising the $\mathrm{S}^{4} \mathrm{G}$ pipeline is presented by Sheth et al. (2010). Here, we make use of the science-ready images produced by our Pipeline 1 . These images are flux-calibrated in units of $\mathrm{MJy} \mathrm{sr}^{-1}$. The FWHM of the point spread function (PSF) is $1^{\prime \prime} .7$, which maps to a physical scale of $170 \mathrm{pc}$ at the median distance of our subsample of 218 galaxies $(21 \mathrm{Mpc})$. Masking all relevant foreground stars and background galaxies is essential in order to obtain reliable profiles that reach low surface brightness levels. Object masks are produced by our Pipeline 2 using SExtractor (Bertin \& Arnouts 1996), and are later visually checked and edited by hand, masking or unmasking pixels as needed.

Here we describe Pipeline 3, which measures the sky level and noise, and obtains radial profiles of surface brightness, position angle (P.A.), and ellipticity $(\epsilon)$. We measure the sky in two concentric and adjacent elliptical rings around the galaxy. The major diameter of the innermost ring is initially set by default to $2 \times D_{25}$, but this value can be modified as needed, depending on the spatial extent of each galaxy and the available background area within the field of view. Each annulus is then azimuthally subdivided into 45 sectors which serve as "sky boxes." These boxes are grown outward, avoiding the masked areas, until they contain 1000 unmasked pixels each. We then compute the median sky value within each box. Should there be any significant difference between the sky value in the

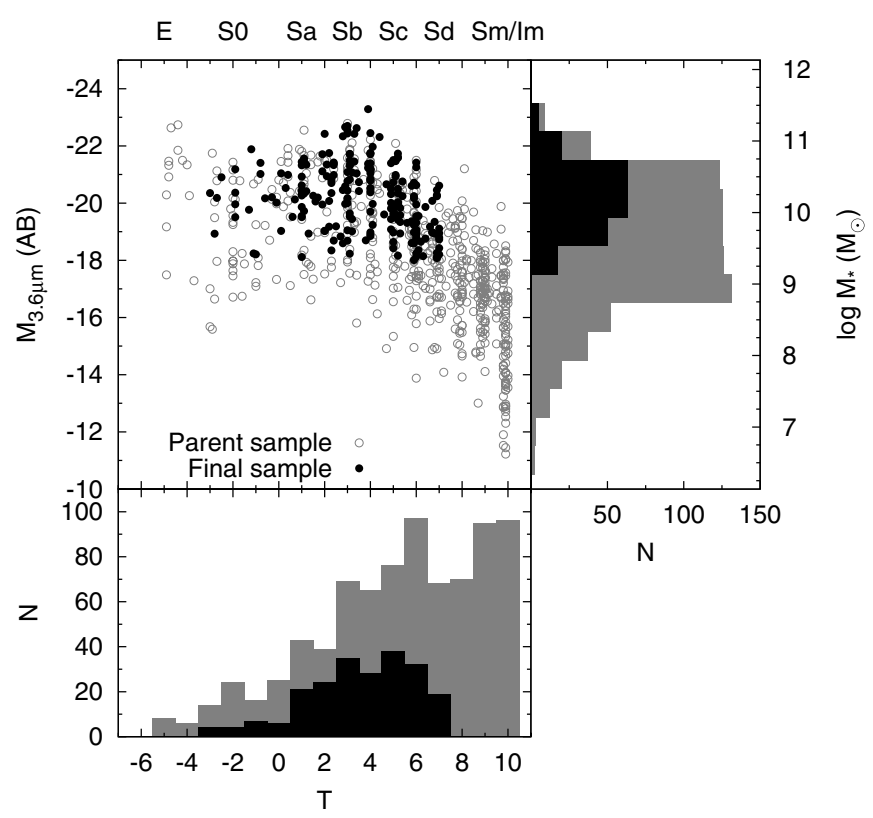

Figure 1. Distribution of Hubble types and absolute magnitudes at $3.6 \mu \mathrm{m}$. The parent sample of 827 galaxies with fully reduced and processed data is shown in gray, whereas the final sample of 218 face-on disks is shown in black.

inner and outer annuli, we readjust the radii of the elliptical annuli accordingly. The goal is to minimize the contamination from the galaxy itself, while still ensuring that the derived background value is representative of the area of the sky where the galaxy lies. In those problematic cases where this method yields unreliable results, the sky boxes are placed by hand.

Taking the galaxy coordinates from LEDA as input, we fit the centroid of each source using the IRAF ${ }^{21}$ task IMCENTROID. Using these new and more accurate central coordinates, we run the task ELLIPSE to get radial profiles of surface brightness, ellipticity, and P.A., keeping the center fixed. We perform a first run with a coarse radial step of $6^{\prime \prime}$, and determine the ellipticity and P.A. at a surface brightness level of 25.5 and 26.5 $\mathrm{AB}$ mag $\operatorname{arcsec}^{-2}$. The values at 25.5 $\mathrm{AB}$ mag arcsec ${ }^{-2}$ are typically robust enough against variations in, e.g., the sky level or the degree of masking, so we adopt them as representative of the shape of the outer parts of our galaxies. A second ELLIPSE run is then performed, keeping the ellipticity and P.A. fixed to these outer values, and using a finer radial increment of $2^{\prime \prime}$ that better matches the FWHM of the PSF. This is the fit that we use to measure disk breaks. A third fit with a step of $2^{\prime \prime}$ but with free ellipticity and P.A. is also performed; we use this third fit to determine the properties of bars (length, ellipticity, and P.A.).

The uncertainty in the surface brightness is computed following the methodology described in Gil de Paz \& Madore (2005) and Muñoz-Mateos et al. (2009). There are two main sources of error at each radius: Poisson noise in the incoming flux and errors in the determination of the sky level. The former is derived by replicating the ellipse measurements on the IRAC coverage maps, to take into account that the effective gain might vary spatially, depending on the mosaicking pattern. The uncertainty in the sky level, on the other hand, comprises pixel-to-pixel noise as well as large-scale background variations. These values are

21 IRAF is distributed by the National Optical Astronomy Observatories, which are operated by the Association of Universities for Research in Astronomy, Inc., under cooperative agreement with the National Science Foundation. 
Table 1

Sample

\begin{tabular}{|c|c|c|c|c|c|c|c|c|c|}
\hline $\begin{array}{l}\text { Galaxy } \\
\text { (1) }\end{array}$ & $\begin{array}{c}\text { R.A. } \\
\text { (hh:mm:ss) } \\
(2)\end{array}$ & $\begin{array}{c}\text { Decl. } \\
\text { (dd:mm:ss) } \\
\text { (3) }\end{array}$ & $\begin{array}{l}\text { Morph. } \\
\text { Type } \\
\text { (4) }\end{array}$ & $\begin{array}{c}T \\
\text { Type } \\
(5)\end{array}$ & $\begin{array}{l}\text { Dist. } \\
(\mathrm{Mpc}) \\
(6)\end{array}$ & $\begin{array}{l}R_{25.5} \\
\left(^{\prime \prime}\right) \\
(7)\end{array}$ & $\begin{array}{l}e_{25.5} \\
(8)\end{array}$ & $\begin{array}{l}\text { P.A.25.5 } \\
\text { (deg) } \\
\text { (9) }\end{array}$ & $\begin{array}{c}M_{3.6 \mu \mathrm{m}} \\
(\mathrm{AB} \mathrm{mag}) \\
(10)\end{array}$ \\
\hline ESO026-001 & 202459.0 & -813435.5 & $\mathrm{SBc}$ & 5.9 & 19 & 57.3 & 0.060 & -61.9 & -18.01 \\
\hline ESO027-001 & 215226.6 & -813151.1 & $\mathrm{SBc}$ & 5.0 & 18 & 103.3 & 0.245 & -9.3 & -19.80 \\
\hline ESO079-007 & 005004.2 & -663307.8 & Scd & 7.0 & 25 & 54.9 & 0.182 & 19.7 & -18.43 \\
\hline ESO234-049 & 203518.1 & -495156.7 & $\mathrm{Sbc}$ & 4.0 & 36 & 59.4 & 0.130 & 58.6 & -19.70 \\
\hline ESO482-035 & 034114.8 & -235013.3 & $\mathrm{Sab}$ & 2.2 & 27 & 72.5 & 0.366 & 1.2 & -19.17 \\
\hline ESO549-018 & 034814.1 & -212827.3 & $\mathrm{SABc}$ & 4.9 & 26 & 87.8 & 0.385 & 15.0 & -19.44 \\
\hline IC0101 & 012408.6 & 095549.4 & $\mathrm{Sb}$ & 3.0 & 44 & 42.2 & 0.464 & -58.8 & -19.05 \\
\hline IC0167 & 015108.6 & 215445.9 & $\mathrm{SABc}$ & 5.0 & 28 & 76.3 & 0.463 & -66.8 & -18.64 \\
\hline IC0749 & 115834.1 & 424402.6 & $\mathrm{Sc}$ & 5.9 & 17 & 76.8 & 0.107 & -52.5 & -18.98 \\
\hline IC0797 & 123154.8 & 150726.9 & $\mathrm{Sc}$ & 6.0 & 18 & 73.6 & 0.347 & -79.4 & -18.24 \\
\hline IC0800 & 123356.7 & 152117.0 & $\mathrm{SBc}$ & 5.2 & 33 & 65.2 & 0.227 & -20.1 & -19.42 \\
\hline IC1158 & 160134.1 & 014228.2 & $\mathrm{SABc}$ & 5.1 & 29 & 82.1 & 0.421 & -48.9 & -19.60 \\
\hline IC1265 & 173639.4 & 420517.7 & $\mathrm{Sab}$ & 2.0 & 31 & 68.4 & 0.424 & 80.0 & -19.02 \\
\hline IC1826 & 023904.0 & -272635.8 & S0/a & -0.6 & 36 & 62.9 & 0.081 & -40.6 & -20.17 \\
\hline IC1933 & 032539.9 & -524707.9 & $\mathrm{Sc}$ & 6.1 & 17 & 70.8 & 0.430 & 54.1 & -18.35 \\
\hline IC1954 & 033131.2 & -515417.4 & $\mathrm{SBb}$ & 3.2 & 14 & 121.8 & 0.441 & 61.0 & -19.44 \\
\hline IC2007 & 035522.8 & -280927.9 & $\mathrm{Sbc}$ & 3.8 & 21 & 49.1 & 0.380 & 53.0 & -18.70 \\
\hline IC2040 & 041259.7 & -323312.2 & $\mathrm{~S} 0 / \mathrm{a}$ & -1.1 & 19 & 48.1 & 0.284 & 65.7 & -18.24 \\
\hline IC2051 & 035200.8 & -834950.7 & $\mathrm{SBbc}$ & 4.0 & 24 & 104.1 & 0.384 & 72.3 & -21.14 \\
\hline IC2056 & 041624.5 & -601224.5 & $\mathrm{SABb}$ & 4.1 & 20 & 60.3 & 0.129 & 16.4 & -19.97 \\
\hline IC4237 & 132432.8 & -210812.8 & $\mathrm{SBb}$ & 3.4 & 40 & 74.3 & 0.252 & -41.9 & -21.04 \\
\hline IC5069 & 210010.4 & -714838.3 & $\mathrm{SBb}$ & 3.2 & 40 & 40.6 & 0.027 & 16.7 & -19.01 \\
\hline IC5332 & 233427.4 & -360603.7 & $\mathrm{SABc}$ & 6.8 & 8.4 & 230.1 & 0.022 & 32.3 & -18.93 \\
\hline NGC 0150 & 003415.5 & -274812.7 & $\mathrm{SBb}$ & 3.5 & 22 & 128.1 & 0.481 & -72.5 & -20.73 \\
\hline NGC 0254 & 004727.6 & -312518.4 & $\mathrm{~S} 0 / \mathrm{a}$ & -1.3 & 17 & 104.4 & 0.348 & -48.3 & -19.77 \\
\hline NGC 0255 & 004747.3 & -112807.4 & $\mathrm{Sbc}$ & 4.1 & 20 & 78.8 & 0.084 & 12.2 & -19.24 \\
\hline NGC 0289 & 005242.3 & -311221.0 & $\mathrm{SBbc}$ & 3.9 & 23 & 114.8 & 0.203 & -61.3 & -21.23 \\
\hline NGC 0300 & 005453.4 & -374103.1 & Scd & 6.9 & 2.0 & 670.2 & 0.328 & -65.7 & -18.08 \\
\hline NGC 0337 & 005950.0 & -073434.4 & SBcd & 6.7 & 20 & 93.9 & 0.293 & -57.9 & -20.08 \\
\hline NGC 0406 & 010724.2 & -695232.1 & $\mathrm{Sc}$ & 4.9 & 21 & 97.9 & 0.494 & -21.5 & -19.08 \\
\hline NGC 0470 & 011944.8 & 032435.7 & $\mathrm{Sb}$ & 3.1 & 40 & 93.8 & 0.482 & -27.2 & -21.71 \\
\hline NGC 0473 & 011955.1 & 163241.2 & S0/a & -0.3 & 30 & 64.5 & 0.338 & -25.0 & -20.20 \\
\hline NGC 0488 & 012146.8 & 051524.5 & $\mathrm{Sb}$ & 2.9 & 29 & 211.2 & 0.175 & 17.6 & -22.66 \\
\hline NGC 0628 & 013641.7 & 154701.1 & $\mathrm{Sc}$ & 5.2 & 8.2 & 332.8 & 0.059 & -48.0 & -20.50 \\
\hline NGC 0658 & 014209.6 & 123606.6 & $\mathrm{Sb}$ & 2.9 & 37 & 85.2 & 0.430 & 26.4 & -20.59 \\
\hline NGC 0685 & 014742.8 & -524543.1 & $\mathrm{Sc}$ & 5.3 & 15 & 132.5 & 0.234 & -78.7 & -19.34 \\
\hline NGC 0691 & 015041.7 & 214536.0 & $\mathrm{Sbc}$ & 4.0 & 36 & 110.9 & 0.249 & -86.3 & -21.39 \\
\hline NGC 0718 & 015313.3 & 041144.7 & $\mathrm{Sa}$ & 1.0 & 21 & 91.7 & 0.109 & 4.7 & -20.31 \\
\hline NGC 0723 & 015345.7 & -234527.8 & $\mathrm{Sbc}$ & 4.0 & 21 & 46.6 & 0.145 & -30.6 & -18.85 \\
\hline NGC 0772 & 015919.5 & 190027.6 & $\mathrm{Sb}$ & 3.0 & 32 & 225.0 & 0.342 & -64.0 & -22.70 \\
\hline NGC 0908 & 022304.5 & -211401.8 & $\mathrm{Sc}$ & 5.1 & 18 & 200.4 & 0.495 & 76.8 & -21.49 \\
\hline NGC 0918 & 022550.8 & 182946.7 & $\mathrm{Sc}$ & 5.2 & 18 & 125.2 & 0.413 & -23.7 & -19.99 \\
\hline NGC 0936 & 022737.4 & -010921.4 & $\mathrm{~S} 0 / \mathrm{a}$ & -1.2 & 21 & 175.1 & 0.247 & -54.4 & -21.88 \\
\hline NGC 0941 & 022827.9 & -010905.3 & $\mathrm{SABc}$ & 5.3 & 21 & 82.3 & 0.277 & -14.8 & -18.94 \\
\hline NGC 0986 & 023334.3 & -390242.0 & $\mathrm{SBab}$ & 2.3 & 17 & 137.6 & 0.104 & -65.3 & -20.87 \\
\hline NGC 0991 & 023532.7 & -070915.6 & $\mathrm{SABc}$ & 5.0 & 19 & 84.8 & 0.054 & -41.1 & -18.97 \\
\hline NGC 1015 & 023811.5 & -011907.6 & $\mathrm{Sa}$ & 1.0 & 33 & 84.9 & 0.056 & -23.1 & -20.62 \\
\hline NGC 1022 & 023832.7 & -064038.7 & $\mathrm{SBa}$ & 1.1 & 18 & 104.7 & 0.094 & 40.3 & -20.33 \\
\hline NGC 1042 & 024024.0 & -082600.7 & $\mathrm{SABc}$ & 6.0 & 10 & 160.1 & 0.212 & 4.7 & -18.96 \\
\hline NGC 1073 & 024340.6 & 012234.4 & $\mathrm{SBc}$ & 5.3 & 15 & 152.2 & 0.143 & 15.1 & -19.58 \\
\hline NGC 1084 & 024559.9 & -073442.5 & $\mathrm{Sc}$ & 4.9 & 21 & 123.0 & 0.237 & 55.2 & -21.40 \\
\hline NGC 1087 & 024625.2 & -002955.8 & $\mathrm{SABc}$ & 5.2 & 17 & 126.6 & 0.366 & 0.8 & -20.35 \\
\hline NGC 1097 & 024618.9 & -301629.0 & $\mathrm{SBb}$ & 3.3 & 17 & 349.1 & 0.331 & -47.0 & -22.42 \\
\hline NGC 1179 & 030238.5 & -185352.6 & $\mathrm{Sc}$ & 5.9 & 18 & 138.6 & 0.221 & 45.6 & -19.23 \\
\hline NGC 1187 & 030237.6 & -225201.6 & $\mathrm{Sc}$ & 5.0 & 18 & 163.8 & 0.231 & -55.1 & -20.73 \\
\hline NGC 1222 & 030856.7 & -025718.6 & $\mathrm{E} / \mathrm{SO}$ & -3.0 & 31 & 88.6 & 0.428 & -10.9 & -20.35 \\
\hline NGC 1232 & 030945.4 & -203444.4 & $\mathrm{SABc}$ & 5.0 & 19 & 227.5 & 0.154 & -84.0 & -21.51 \\
\hline NGC 1255 & 031332.0 & -254330.3 & $\mathrm{SABb}$ & 4.0 & 22 & 132.2 & 0.272 & -56.3 & -20.53 \\
\hline NGC 1258 & 031405.5 & -214627.8 & $\mathrm{SABc}$ & 5.8 & 26 & 50.6 & 0.261 & 16.3 & -18.60 \\
\hline NGC 1299 & 032009.7 & -061543.2 & $\mathrm{SBb}$ & 2.9 & 33 & 48.9 & 0.394 & 51.6 & -19.97 \\
\hline NGC 1300 & 031941.0 & -192439.9 & $\mathrm{Sbc}$ & 4.0 & 19 & 208.3 & 0.101 & -40.8 & -21.20 \\
\hline NGC 1302 & 031951.2 & -260338.0 & S0/a & 0.1 & 20 & 152.4 & 0.069 & -4.1 & -21.04 \\
\hline NGC 1306 & 032103.0 & -253044.8 & $\mathrm{Sb}$ & 2.8 & 20 & 37.5 & 0.207 & -30.7 & -18.59 \\
\hline NGC 1325A & 032448.5 & -212011.9 & $\mathrm{SABc}$ & 6.9 & 18 & 73.5 & 0.096 & 42.8 & -18.26 \\
\hline
\end{tabular}


Table 1

(Continued)

\begin{tabular}{|c|c|c|c|c|c|c|c|c|c|}
\hline $\begin{array}{l}\text { Galaxy } \\
\text { (1) }\end{array}$ & $\begin{array}{c}\text { R.A. } \\
\text { (hh:mm:ss) } \\
(2)\end{array}$ & $\begin{array}{c}\text { Decl. } \\
\text { (dd:mm:ss) } \\
\text { (3) }\end{array}$ & $\begin{array}{l}\text { Morph. } \\
\text { Type } \\
(4)\end{array}$ & $\begin{array}{c}T \\
\text { Type } \\
(5)\end{array}$ & $\begin{array}{l}\text { Dist. } \\
(\mathrm{Mpc}) \\
(6)\end{array}$ & $\begin{array}{l}R_{25.5} \\
\left(^{\prime \prime}\right) \\
(7)\end{array}$ & $\begin{array}{l}e_{25.5} \\
(8)\end{array}$ & $\begin{array}{c}\text { P.A.25.5 } \\
\text { (deg) } \\
(9)\end{array}$ & $\begin{array}{c}M_{3.6 \mu \mathrm{m}} \\
(\mathrm{AB} \mathrm{mag}) \\
(10)\end{array}$ \\
\hline NGC 1326 & 032356.4 & -362752.3 & $\mathrm{~S} 0 / \mathrm{a}$ & -0.8 & 17 & 164.0 & 0.259 & 68.1 & -21.02 \\
\hline NGC 1338 & 032854.5 & -120912.1 & $\mathrm{SABb}$ & 3.0 & 35 & 50.8 & 0.124 & 10.2 & -20.17 \\
\hline NGC 1341 & 032758.4 & -370900.6 & $\mathrm{Sa}$ & 1.3 & 17 & 61.6 & 0.057 & -9.1 & -18.93 \\
\hline NGC 1347 & 032941.8 & -221645.4 & $\mathrm{SBc}$ & 5.0 & 25 & 47.7 & 0.088 & -21.3 & -18.42 \\
\hline NGC 1350 & 033108.1 & -333742.0 & $\mathrm{Sab}$ & 1.9 & 21 & 248.3 & 0.477 & 5.3 & -21.71 \\
\hline NGC 1357 & 033317.0 & -133950.9 & $\mathrm{Sab}$ & 1.9 & 25 & 143.1 & 0.240 & 77.9 & -21.11 \\
\hline NGC 1367 & 033501.3 & -245559.5 & $\mathrm{Sa}$ & 1.0 & 22 & 194.8 & 0.334 & -46.8 & -21.44 \\
\hline NGC 1385 & 033728.5 & -243004.3 & $\mathrm{SBc}$ & 5.9 & 15 & 131.7 & 0.329 & -3.8 & -20.17 \\
\hline NGC 1398 & 033852.1 & -262015.6 & SBab & 2.0 & 20 & 286.1 & 0.242 & -84.4 & -22.42 \\
\hline NGC 1433 & 034201.5 & -471319.1 & $\mathrm{SBa}$ & 1.5 & 10.0 & 198.3 & 0.087 & -164.9 & -20.26 \\
\hline NGC 1436 & 034337.1 & -355111.0 & $\mathrm{Sab}$ & 1.9 & 19 & 114.5 & 0.278 & -34.2 & -19.95 \\
\hline NGC 1438 & 034517.2 & -230008.8 & $\mathrm{Sa}$ & 0.7 & 29 & 74.5 & 0.472 & 71.1 & -20.13 \\
\hline NGC 1452 & 034522.3 & -183801.3 & S0/a & 0.3 & 23 & 95.6 & 0.365 & -66.3 & -20.53 \\
\hline NGC 1493 & 035727.4 & -461238.6 & $\mathrm{SBc}$ & 6.0 & 11 & 120.3 & 0.080 & -85.3 & -18.80 \\
\hline NGC 1494 & 035742.4 & -485430.1 & $\mathrm{SABc}$ & 7.0 & 15 & 120.8 & 0.417 & -3.6 & -18.73 \\
\hline NGC 1512 & 040354.1 & -432055.5 & $\mathrm{SBa}$ & 1.2 & 12 & 225.4 & 0.263 & 73.8 & -20.41 \\
\hline NGC 1533 & 040951.8 & -560706.6 & $\mathrm{E} / \mathrm{SO}$ & -2.5 & 18 & 116.8 & 0.031 & -41.2 & -20.90 \\
\hline NGC 1559 & 041735.7 & -624700.9 & $\mathrm{SBc}$ & 5.9 & 15 & 139.1 & 0.389 & 66.2 & -20.71 \\
\hline NGC 1566 & 042000.4 & -545616.8 & $\mathrm{SABb}$ & 4.0 & 12 & 264.0 & 0.152 & -89.0 & -21.04 \\
\hline NGC 1637 & 044128.2 & -025128.7 & $\mathrm{Sc}$ & 5.0 & 11 & 156.7 & 0.277 & 25.5 & -19.63 \\
\hline NGC 1640 & 044214.5 & -202605.4 & $\mathrm{Sb}$ & 3.0 & 19 & 95.4 & 0.106 & 53.8 & -20.00 \\
\hline NGC 1672 & 044542.5 & -591450.2 & $\mathrm{Sb}$ & 3.3 & 14 & 239.1 & 0.113 & -28.6 & -21.45 \\
\hline NGC 1688 & 044823.7 & -594801.0 & $\mathrm{SBc}$ & 6.2 & 13 & 98.2 & 0.351 & -0.5 & -18.65 \\
\hline NGC 1703 & 045252.1 & -594432.4 & $\mathrm{SBb}$ & 3.1 & 17 & 97.1 & 0.094 & -59.9 & -19.64 \\
\hline NGC 1792 & 050514.4 & -375850.1 & SBbc & 4.0 & 13 & 186.9 & 0.451 & -45.5 & -21.18 \\
\hline NGC 1808 & 050742.3 & -373046.2 & $\mathrm{SABa}$ & 1.2 & 12 & 273.8 & 0.278 & -60.8 & -21.34 \\
\hline NGC 2500 & 080153.2 & 504413.9 & SBcd & 7.0 & 10 & 106.5 & 0.133 & 62.7 & -18.20 \\
\hline NGC 2633 & 084804.6 & 740556.3 & $\mathrm{SBb}$ & 3.0 & 30 & 80.2 & 0.293 & 4.4 & -21.09 \\
\hline NGC 2805 & 092020.4 & 640610.7 & $\mathrm{SABc}$ & 6.9 & 28 & 146.7 & 0.208 & -42.4 & -20.46 \\
\hline NGC 2841 & 092202.7 & 505835.7 & $\mathrm{Sb}$ & 3.0 & 18 & 310.5 & 0.481 & -30.1 & -22.54 \\
\hline NGC 2844 & 092148.0 & 400904.6 & $\mathrm{Sa}$ & 0.6 & 24 & 71.8 & 0.439 & 11.6 & -19.52 \\
\hline NGC 2906 & 093206.2 & 082630.6 & $\mathrm{Sc}$ & 5.9 & 30 & 65.7 & 0.372 & 82.2 & -20.67 \\
\hline NGC 2964 & 094254.2 & 315050.5 & $\mathrm{Sbc}$ & 4.0 & 21 & 108.7 & 0.401 & -82.8 & -20.79 \\
\hline NGC 3032 & 095208.2 & 291410.4 & So & -1.9 & 22 & 74.9 & 0.227 & -80.6 & -19.51 \\
\hline NGC 3049 & 095449.6 & 091616.3 & SBab & 2.5 & 21 & 77.2 & 0.329 & 28.8 & -19.19 \\
\hline NGC 3073 & 100052.1 & 553707.9 & $\mathrm{E} / \mathrm{SO}$ & -2.8 & 26 & 63.8 & 0.170 & 84.8 & -18.93 \\
\hline NGC 3147 & 101653.7 & 732402.7 & $\mathrm{Sbc}$ & 3.9 & 43 & 150.1 & 0.121 & -35.9 & -23.28 \\
\hline NGC 3184 & 101816.9 & 412527.6 & $\mathrm{SABc}$ & 6.0 & 12 & 239.1 & 0.090 & -44.3 & -20.63 \\
\hline NGC 3206 & 102147.6 & 565549.8 & $\mathrm{SBc}$ & 6.0 & 21 & 80.5 & 0.282 & 7.6 & -18.69 \\
\hline NGC 3319 & 103909.5 & 414112.6 & $\mathrm{SBc}$ & 6.0 & 14 & 180.5 & 0.432 & 42.3 & -18.90 \\
\hline NGC 3344 & 104331.1 & 245520.7 & $\mathrm{Sbc}$ & 4.0 & 6.1 & 187.8 & 0.096 & -59.2 & -18.91 \\
\hline NGC 3351 & 104357.7 & 114213.2 & $\mathrm{Sb}$ & 3.1 & 10 & 253.2 & 0.260 & 11.6 & -20.83 \\
\hline NGC 3368 & 104645.7 & 114911.9 & $\mathrm{SABa}$ & 2.1 & 11 & 345.1 & 0.385 & -7.9 & -21.35 \\
\hline NGC 3486 & 110023.9 & 285830.3 & $\mathrm{Sc}$ & 5.2 & 12 & 203.8 & 0.275 & 85.4 & -19.90 \\
\hline NGC 3504 & 110311.2 & 275821.5 & $\mathrm{SABa}$ & 2.1 & 20 & 106.0 & 0.066 & -41.7 & -20.94 \\
\hline NGC 3627 & 112015.0 & 125929.4 & $\mathrm{SABb}$ & 3.1 & 10 & 393.4 & 0.458 & -7.0 & -21.72 \\
\hline NGC 3726 & 113321.1 & 470145.3 & $\mathrm{Sc}$ & 5.1 & 17 & 197.7 & 0.387 & 19.4 & -20.94 \\
\hline NGC 3794 & 114054.2 & 561207.5 & $\mathrm{SABc}$ & 6.3 & 20 & 77.2 & 0.437 & -62.6 & -18.14 \\
\hline NGC 3938 & 115249.5 & 440714.7 & $\mathrm{Sc}$ & 5.1 & 17 & 166.9 & 0.076 & 20.9 & -20.98 \\
\hline NGC 3953 & 115349.0 & 521936.5 & $\mathrm{Sbc}$ & 4.0 & 18 & 212.7 & 0.481 & 12.6 & -21.75 \\
\hline NGC 4051 & 120309.6 & 443152.7 & $\mathrm{SABb}$ & 4.0 & 15 & 198.3 & 0.247 & -54.7 & -20.85 \\
\hline NGC 4136 & 120917.7 & 295539.2 & $\mathrm{Sc}$ & 5.2 & 9.7 & 110.9 & 0.058 & -43.9 & -18.16 \\
\hline NGC 4245 & 121736.8 & 293628.8 & $\mathrm{~S} 0 / \mathrm{a}$ & 0.1 & 9.7 & 118.1 & 0.172 & 0.5 & -19.03 \\
\hline NGC 4254 & 121849.6 & 142459.1 & $\mathrm{Sc}$ & 5.2 & 15 & 191.9 & 0.195 & 84.1 & -21.58 \\
\hline NGC 4262 & 121930.6 & 145239.6 & $\mathrm{E} / \mathrm{SO}$ & -2.7 & 18 & 72.4 & 0.094 & -6.0 & -20.18 \\
\hline NGC 4298 & 122132.8 & 143622.0 & $\mathrm{Sc}$ & 5.2 & 15 & 156.0 & 0.410 & -50.4 & -20.02 \\
\hline NGC 4303 & 122154.9 & 042825.5 & $\mathrm{SABb}$ & 4.0 & 12 & 213.2 & 0.092 & -27.0 & -21.15 \\
\hline NGC 4314 & 122232.0 & 295343.6 & $\mathrm{SBa}$ & 1.0 & 9.7 & 157.9 & 0.078 & 42.7 & -19.87 \\
\hline NGC 4319 & 122143.9 & 751921.0 & SBab & 2.4 & 28 & 118.5 & 0.209 & -36.6 & -20.98 \\
\hline NGC 4321 & 122254.9 & 154920.3 & $\mathrm{SABb}$ & 4.1 & 16 & 312.7 & 0.228 & -8.2 & -21.97 \\
\hline NGC 4351 & 122401.5 & 121217.6 & SBab & 2.4 & 18 & 93.7 & 0.325 & 62.5 & -18.69 \\
\hline NGC 4355 & 122654.6 & -005239.6 & $\mathrm{SABa}$ & 1.1 & 31 & 59.5 & 0.489 & 62.9 & -19.72 \\
\hline NGC 4369 & 122436.2 & 392258.6 & $\mathrm{Sa}$ & 1.0 & 22 & 81.2 & 0.021 & 74.8 & -20.26 \\
\hline NGC 4380 & 122522.2 & 100100.5 & Sab & 2.3 & 20 & 144.2 & 0.444 & -23.8 & -20.35 \\
\hline
\end{tabular}


Table 1

(Continued)

\begin{tabular}{|c|c|c|c|c|c|c|c|c|c|}
\hline $\begin{array}{l}\text { Galaxy } \\
\text { (1) }\end{array}$ & $\begin{array}{c}\text { R.A. } \\
\text { (hh:mm:ss) } \\
(2)\end{array}$ & $\begin{array}{c}\text { Decl. } \\
\text { (dd:mm:ss) } \\
\text { (3) }\end{array}$ & $\begin{array}{l}\text { Morph. } \\
\text { Type } \\
(4)\end{array}$ & $\begin{array}{c}T \\
\text { Type } \\
(5)\end{array}$ & $\begin{array}{l}\text { Dist. } \\
(\mathrm{Mpc}) \\
(6)\end{array}$ & $\begin{array}{l}R_{25.5} \\
\left(^{\prime \prime}\right) \\
(7)\end{array}$ & $\begin{array}{l}e_{25.5} \\
(8)\end{array}$ & $\begin{array}{l}\text { P.A.25.5 } \\
\text { (deg) } \\
(9)\end{array}$ & $\begin{array}{c}M_{3.6 \mu \mathrm{m}} \\
(\mathrm{AB} \mathrm{mag}) \\
(10)\end{array}$ \\
\hline NGC 4394 & 122555.6 & 181250.2 & $\mathrm{SBb}$ & 3.0 & 17 & 145.4 & 0.165 & -74.9 & -20.44 \\
\hline NGC 4405 & 122607.1 & 161051.8 & $\mathrm{~S} 0 / \mathrm{a}$ & -0.1 & 25 & 80.2 & 0.255 & 16.4 & -20.02 \\
\hline NGC 4413 & 122632.3 & 123641.8 & Sab & 2.0 & 17 & 99.9 & 0.309 & 64.8 & -19.03 \\
\hline NGC 4424 & 122711.6 & 092514.0 & $\mathrm{SBa}$ & 1.0 & 16 & 146.9 & 0.472 & 87.4 & -19.51 \\
\hline NGC 4450 & 122829.6 & 170505.9 & $\mathrm{Sab}$ & 2.4 & 17 & 210.8 & 0.310 & -7.2 & -21.38 \\
\hline NGC 4457 & 122859.0 & 033414.3 & $\mathrm{~S} 0 / \mathrm{a}$ & 0.4 & 17 & 158.6 & 0.047 & 69.7 & -20.99 \\
\hline NGC 4498 & 123139.5 & 165109.9 & $\mathrm{Sc}$ & 6.4 & 16 & 99.6 & 0.455 & -39.9 & -18.76 \\
\hline NGC 4501 & 123159.2 & 142513.2 & $\mathrm{Sb}$ & 3.4 & 20 & 285.3 & 0.476 & -39.1 & -22.62 \\
\hline NGC 4548 & 123526.5 & 142946.8 & $\mathrm{Sb}$ & 3.1 & 16 & 218.1 & 0.147 & -35.8 & -21.32 \\
\hline NGC 4579 & 123743.5 & 114905.5 & $\mathrm{SABb}$ & 2.8 & 20 & 248.7 & 0.271 & -83.5 & -22.34 \\
\hline NGC 4580 & 123748.4 & 052206.7 & $\mathrm{SABa}$ & 1.6 & 21 & 91.4 & 0.251 & -25.8 & -20.06 \\
\hline NGC 4639 & 124252.4 & 131526.8 & $\mathrm{Sbc}$ & 3.5 & 22 & 99.1 & 0.360 & -51.6 & -20.42 \\
\hline NGC 4651 & 124342.6 & 162336.1 & $\mathrm{Sc}$ & 5.2 & 27 & 153.7 & 0.328 & 71.7 & -21.73 \\
\hline NGC 4689 & 124745.6 & 134545.9 & $\mathrm{Sc}$ & 4.7 & 18 & 184.8 & 0.182 & -15.6 & -20.66 \\
\hline NGC 4713 & 124957.9 & 051840.9 & Scd & 6.8 & 17 & 83.1 & 0.260 & -82.0 & -19.35 \\
\hline NGC 4725 & 125026.6 & 253002.5 & $\mathrm{SABa}$ & 2.2 & 14 & 372.4 & 0.373 & 36.0 & -21.75 \\
\hline NGC 4750 & 125007.3 & 725228.7 & $\mathrm{Sab}$ & 2.4 & 26 & 117.4 & 0.232 & -10.8 & -21.42 \\
\hline NGC 4772 & 125329.2 & 021006.0 & $\mathrm{Sa}$ & 1.1 & 30 & 158.6 & 0.456 & -32.4 & -21.46 \\
\hline NGC 4793 & 125440.6 & 285619.4 & $\mathrm{Sc}$ & 5.1 & 34 & 91.9 & 0.395 & 39.6 & -21.47 \\
\hline NGC 4806 & 125612.4 & -293010.1 & $\mathrm{Sc}$ & 4.9 & 34 & 45.5 & 0.165 & 29.2 & -19.81 \\
\hline NGC 4826 & 125643.6 & 214059.1 & $\mathrm{Sab}$ & 2.3 & 5.4 & 356.5 & 0.467 & -64.9 & -20.70 \\
\hline NGC 4942 & 130419.1 & -073858.4 & $\mathrm{SABc}$ & 7.0 & 28 & 56.3 & 0.184 & -33.5 & -19.11 \\
\hline NGC 4980 & 130910.1 & -283830.4 & $\mathrm{SABa}$ & 1.0 & 20 & 61.3 & 0.486 & -10.1 & -18.11 \\
\hline NGC 4984 & 130857.3 & -153058.6 & $\mathrm{~S} 0 / \mathrm{a}$ & -0.8 & 21 & 183.9 & 0.393 & 29.8 & -21.41 \\
\hline NGC 5055 & 131549.3 & 420145.7 & $\mathrm{Sbc}$ & 4.0 & 9.0 & 516.9 & 0.395 & -76.5 & -21.74 \\
\hline NGC 5068 & 131854.7 & -210219.4 & $\mathrm{Sc}$ & 6.0 & 6.1 & 227.7 & 0.011 & -75.5 & -19.07 \\
\hline NGC 5240 & 133555.2 & 353517.6 & $\mathrm{SBc}$ & 5.8 & 49 & 64.4 & 0.346 & 61.2 & -20.60 \\
\hline NGC 5248 & 133732.0 & 085306.7 & $\mathrm{SABb}$ & 4.0 & 19 & 207.0 & 0.236 & -74.5 & -21.71 \\
\hline NGC 5273 & 134208.4 & 353915.4 & S0 & -1.9 & 18 & 95.9 & 0.101 & 14.6 & -19.96 \\
\hline NGC 5339 & 135400.3 & -075550.4 & $\mathrm{SBa}$ & 1.0 & 39 & 82.9 & 0.205 & 34.8 & -20.93 \\
\hline NGC 5371 & 135540.0 & 402742.3 & $\mathrm{Sbc}$ & 4.0 & 35 & 139.3 & 0.209 & 16.7 & -22.45 \\
\hline NGC 5377 & 135616.7 & 471408.3 & $\mathrm{Sa}$ & 1.0 & 28 & 159.9 & 0.455 & 26.6 & -21.32 \\
\hline NGC 5468 & 140634.9 & -052710.9 & $\mathrm{SABc}$ & 6.0 & 46 & 88.8 & 0.066 & -86.4 & -21.42 \\
\hline NGC 5480 & 140621.6 & 504330.3 & $\mathrm{Sc}$ & 4.9 & 27 & 78.1 & 0.219 & 39.5 & -20.31 \\
\hline NGC 5584 & 142223.8 & -002315.6 & $\mathrm{SABc}$ & 6.0 & 23 & 117.0 & 0.255 & -20.4 & -19.95 \\
\hline NGC 5585 & 141948.1 & 564345.8 & $\mathrm{SABc}$ & 6.9 & 9.0 & 162.5 & 0.384 & 36.4 & -18.11 \\
\hline NGC 5597 & 142427.4 & -164546.4 & $\mathrm{Sc}$ & 6.0 & 39 & 96.3 & 0.279 & -50.1 & -21.26 \\
\hline NGC 5645 & 143039.3 & 071630.4 & SBcd & 6.6 & 20 & 83.7 & 0.345 & 70.7 & -19.17 \\
\hline NGC 5668 & 143324.3 & 042701.7 & Scd & 6.9 & 27 & 103.1 & 0.142 & -41.9 & -20.24 \\
\hline NGC 5669 & 143243.9 & 095330.8 & $\mathrm{SABc}$ & 6.0 & 20 & 116.3 & 0.251 & 59.4 & -19.55 \\
\hline NGC 5713 & 144011.4 & -001720.0 & $\mathrm{SABb}$ & 4.0 & 24 & 101.5 & 0.130 & 14.8 & -21.18 \\
\hline NGC 5740 & 144424.4 & 014047.3 & $\mathrm{SABb}$ & 3.0 & 29 & 89.5 & 0.453 & -17.3 & -20.77 \\
\hline NGC 5762 & 144842.6 & 122726.0 & $\mathrm{Sb}$ & 3.0 & 25 & 54.5 & 0.168 & -72.3 & -18.68 \\
\hline NGC 5806 & 150000.4 & 015328.9 & $\mathrm{Sb}$ & 3.2 & 25 & 142.5 & 0.473 & -15.6 & -21.07 \\
\hline NGC 5850 & 150707.7 & 013239.4 & $\mathrm{Sb}$ & 3.1 & 23 & 188.0 & 0.228 & -24.6 & -21.32 \\
\hline NGC 5892 & 151348.2 & -152749.7 & $\mathrm{SABc}$ & 7.0 & 34 & 104.8 & 0.125 & -76.9 & -20.61 \\
\hline NGC 5915 & 152133.1 & -130530.3 & SBab & 2.3 & 34 & 56.1 & 0.256 & 16.2 & -20.79 \\
\hline NGC 5949 & 152800.7 & 644547.4 & $\mathrm{Sbc}$ & 4.0 & 15 & 78.2 & 0.402 & -34.6 & -18.98 \\
\hline NGC 5950 & 153130.8 & 402548.3 & $\mathrm{Sb}$ & 3.1 & 38 & 55.3 & 0.464 & 44.7 & -19.53 \\
\hline NGC 5958 & 153449.1 & 283918.9 & $\mathrm{Sc}$ & 4.6 & 28 & 57.7 & 0.159 & 38.2 & -19.60 \\
\hline NGC 5964 & 153736.2 & 055827.3 & SBcd & 6.9 & 27 & 140.6 & 0.275 & -32.3 & -20.28 \\
\hline NGC 5985 & 153937.1 & 591955.5 & $\mathrm{Sb}$ & 3.0 & 44 & 158.1 & 0.491 & 14.6 & -22.43 \\
\hline NGC 6012 & 155413.9 & 143604.3 & SBab & 1.7 & 26 & 92.9 & 0.131 & 17.0 & -20.20 \\
\hline NGC 6014 & 155557.4 & 055555.0 & So & -1.9 & 35 & 67.4 & 0.201 & -37.6 & -20.36 \\
\hline NGC 6063 & 160713.0 & 075844.4 & $\mathrm{Sc}$ & 5.9 & 46 & 56.4 & 0.454 & -27.9 & -20.24 \\
\hline NGC 6106 & 161847.2 & 072439.2 & $\mathrm{Sc}$ & 5.3 & 25 & 79.9 & 0.453 & -38.7 & -19.83 \\
\hline NGC 6140 & 162058.0 & 652326.3 & $\mathrm{Sc}$ & 5.6 & 19 & 109.7 & 0.144 & -79.4 & -19.31 \\
\hline NGC 6155 & 162608.3 & 482200.5 & $\mathrm{Sc}$ & 5.4 & 34 & 52.5 & 0.207 & -41.6 & -20.24 \\
\hline NGC 6181 & 163221.0 & 194935.2 & $\mathrm{SABc}$ & 5.2 & 34 & 79.4 & 0.444 & -6.7 & -21.60 \\
\hline NGC 6207 & 164303.7 & 364955.8 & $\mathrm{Sc}$ & 4.9 & 20 & 93.3 & 0.361 & 19.0 & -20.01 \\
\hline NGC 6217 & 163239.2 & 781153.4 & $\mathrm{Sbc}$ & 4.0 & 24 & 95.6 & 0.049 & 75.1 & -20.83 \\
\hline NGC 6267 & 165808.7 & 225906.5 & $\mathrm{Sc}$ & 4.9 & 42 & 71.6 & 0.496 & 42.6 & -20.50 \\
\hline NGC 6278 & 170050.3 & 230039.7 & S0 & -1.9 & 39 & 65.0 & 0.332 & -54.2 & -21.19 \\
\hline NGC 6339 & 171706.5 & 405041.9 & $\mathrm{Sc}$ & 6.4 & 31 & 93.4 & 0.396 & 3.8 & -19.86 \\
\hline
\end{tabular}


Table 1

(Continued)

\begin{tabular}{|c|c|c|c|c|c|c|c|c|c|}
\hline Galaxy & $\begin{array}{c}\text { R.A. } \\
\text { (hh:mm:ss) } \\
\text { (2) }\end{array}$ & $\begin{array}{c}\text { Decl. } \\
\text { (dd:mm:ss) } \\
\text { (3) }\end{array}$ & $\begin{array}{l}\text { Morph. } \\
\text { Type } \\
\text { (4) }\end{array}$ & $\begin{array}{c}T \\
\text { Type } \\
(5)\end{array}$ & $\begin{array}{l}\text { Dist. } \\
(\mathrm{Mpc}) \\
(6)\end{array}$ & $\begin{array}{l}R_{25.5} \\
\left({ }^{\prime \prime}\right) \\
(7)\end{array}$ & $\begin{array}{l}e_{25.5} \\
(8)\end{array}$ & $\begin{array}{l}\text { P.A.25.5 } \\
\text { (deg) } \\
\text { (9) }\end{array}$ & $\begin{array}{c}M_{3.6 \mu \mathrm{m}} \\
(\mathrm{AB} \mathrm{mag}) \\
(10)\end{array}$ \\
\hline NGC 6412 & 172937.3 & 754216.1 & $\mathrm{SABc}$ & 5.2 & 24 & 80.8 & 0.024 & -62.8 & -20.03 \\
\hline NGC 6434 & 173648.7 & 720520.1 & $\mathrm{SBbc}$ & 4.0 & 38 & 58.1 & 0.380 & -75.7 & -20.38 \\
\hline NGC 6923 & 203139.1 & -304954.6 & $\mathrm{SBb}$ & 3.1 & 37 & 86.6 & 0.344 & 77.9 & -21.50 \\
\hline NGC 7098 & 214416.2 & -750640.6 & $\mathrm{Sa}$ & 1.1 & 29 & 162.5 & 0.421 & 71.9 & -21.56 \\
\hline NGC 7140 & 215215.3 & -553410.9 & $\mathrm{SABb}$ & 3.9 & 37 & 136.2 & 0.359 & 4.0 & -21.38 \\
\hline NGC 7479 & 230456.6 & 121922.5 & $\mathrm{SBbc}$ & 4.4 & 34 & 140.6 & 0.252 & 17.2 & -22.31 \\
\hline NGC 7552 & 231610.7 & -423505.0 & SBab & 2.4 & 17 & 118.6 & 0.037 & 26.0 & -21.33 \\
\hline NGC 7625 & 232030.1 & 171331.6 & $\mathrm{Sa}$ & 1.0 & 23 & 59.9 & 0.091 & -22.4 & -20.44 \\
\hline NGC 7661 & 232714.5 & -651618.8 & $\mathrm{SBc}$ & 5.9 & 32 & 58.4 & 0.375 & 31.8 & -18.58 \\
\hline NGC 7731 & 234129.1 & 034424.0 & $\mathrm{SBa}$ & 1.0 & 37 & 46.2 & 0.265 & 87.8 & -19.55 \\
\hline NGC 7741 & 234354.3 & 260434.0 & $\mathrm{SBc}$ & 6.0 & 14 & 123.5 & 0.224 & -9.0 & -19.31 \\
\hline NGC 7742 & 234415.7 & 104601.5 & $\mathrm{Sb}$ & 2.8 & 22 & 80.7 & 0.028 & -82.0 & -20.46 \\
\hline NGC 7798 & 235925.5 & 204459.3 & $\mathrm{Sc}$ & 5.4 & 33 & 55.3 & 0.140 & 50.4 & -20.75 \\
\hline PGC003853 & 010504.9 & -061244.8 & $\mathrm{SABc}$ & 7.0 & 13 & 158.2 & 0.257 & -75.8 & -18.95 \\
\hline PGC006667 & 014910.3 & -100340.9 & Scd & 6.7 & 25 & 80.2 & 0.219 & -51.0 & -18.98 \\
\hline PGC011367 & 030031.7 & -154410.3 & Scd & 7.0 & 22 & 65.7 & 0.093 & -13.2 & -18.80 \\
\hline PGC012633 & 032217.5 & -070526.4 & $\mathrm{Sab}$ & 2.2 & 38 & 47.4 & 0.024 & 52.6 & -20.07 \\
\hline PGC012664 & 032255.1 & -111212.1 & Scd & 6.7 & 35 & 65.6 & 0.310 & -24.0 & -18.98 \\
\hline PGC014037 & 035318.2 & -102648.0 & $\mathrm{~S} 0 / \mathrm{a}$ & -1.0 & 36 & 35.6 & 0.478 & -41.4 & -18.21 \\
\hline UGC00313 & 003126.0 & 061224.5 & $\mathrm{Sab}$ & 2.3 & 29 & 37.8 & 0.376 & 15.0 & -18.35 \\
\hline UGC01551 & 020337.6 & 240430.7 & $\mathrm{SBc}$ & 6.1 & 36 & 78.1 & 0.229 & -72.5 & -19.55 \\
\hline UGC02443 & 025821.5 & -020230.9 & $\mathrm{Sc}$ & 5.8 & 38 & 50.6 & 0.463 & -19.5 & -19.37 \\
\hline UGC03070 & 043059.7 & -020011.9 & $\mathrm{SABb}$ & 3.1 & 33 & 46.0 & 0.430 & -6.4 & -18.23 \\
\hline UGC09356 & 143253.5 & 113542.0 & $\mathrm{Sb}$ & 2.7 & 31 & 56.6 & 0.363 & -83.3 & -18.83 \\
\hline UGC10437 & 163107.6 & 432054.4 & $\mathrm{Sc}$ & 6.0 & 37 & 49.2 & 0.404 & -17.6 & -18.12 \\
\hline UGC10445 & 163347.6 & 285905.4 & $\mathrm{SBc}$ & 6.0 & 29 & 67.6 & 0.238 & -54.6 & -18.73 \\
\hline
\end{tabular}

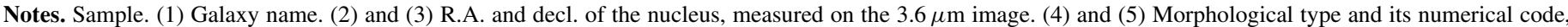

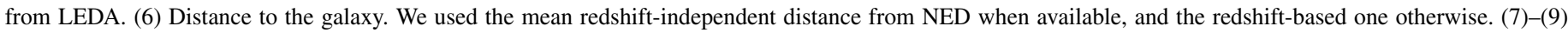

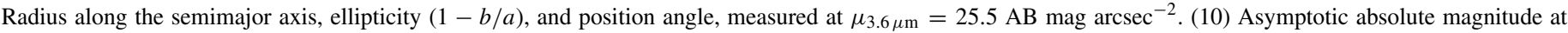
$3.6 \mu \mathrm{m}$.

(This table is also available in a machine-readable form in the online journal.)

computed from the rms within and among the different sky boxes, respectively. Large-scale variations constitute the dominant source of error in the outskirts of our galaxies (see also Comerón et al. 2011b; Martín-Navarro et al. 2012).

An additional correction needs to be applied to the surface photometry, in order to account for both the extended emission of the PSF and the scattered light on the detector. According to the IRAC handbook, ${ }^{22}$ if $F_{\text {obs }}$ is the total flux inside an elliptical aperture with major and minor radii $a$ and $b$, then the aperturecorrected flux can be obtained as

$$
F_{\text {corr }}\left(r_{\mathrm{eq}}\right)=F_{\mathrm{obs}}\left(r_{\mathrm{eq}}\right) \times\left(A e^{-r_{\mathrm{eq}}^{B}}+C\right),
$$

where $r_{\mathrm{eq}}=\sqrt{a b}$ is the equivalent radius of the aperture in arcseconds, and the constants $A, B$, and $C$ are equal to $0.82,0.370$, and 0.910 , respectively, for the $3.6 \mu \mathrm{m}$ band. The uncertainty in this correction is estimated to lie within 5\%-10\%.

Again, this expression is only valid for the total flux inside an aperture. In order to obtain an analogous expression for the surface brightness along a given isophote, $I_{\text {corr }}$, we can simply apply a series expansion to Equation (1):

$$
\begin{aligned}
I_{\mathrm{corr}}\left(r_{\mathrm{eq}}\right)= & I_{\mathrm{obs}}\left(r_{\mathrm{eq}}\right) \times\left(A e^{-r_{\mathrm{eq}}^{B}}+C\right) \\
& -A B r_{\mathrm{eq}}^{B-2} e^{-r_{\mathrm{eq}}^{B}} F_{\mathrm{obs}}\left(r_{\mathrm{eq}}\right) /(2 \pi) .
\end{aligned}
$$

\footnotetext{
22 http://irsa.ipac.caltech.edu/data/SPITZER/docs/irac/ iracinstrumenthandbook/30/
}

Note that for large apertures, $F_{\text {corr }} \simeq C \times F_{\text {obs }}$ and $I_{\text {corr }} \simeq$ $C \times I_{\text {obs }}$, but here we explicitly use Equations (1) and (2) at each radius.

The surface brightness can be then computed as

$$
\mu_{\mathrm{corr}}\left(\mathrm{AB} \text { mag } \operatorname{arcsec}^{-2}\right)=-2.5 \log \left[I_{\mathrm{corr}}\left(\mathrm{MJy} \mathrm{str}^{-1}\right)\right]+21.097 \text {, }
$$

where the zero point has been computed according to the standard definition of the AB magnitude scale (Oke 1974).

As a byproduct of the surface photometry, we also obtain the asymptotic magnitude for each galaxy, that is, the magnitude that we would measure with a hypothetically infinite aperture. We do so by calculating $m(r)$, the total magnitude within a radius $r$, as a function of the local magnitude gradient, $d m(r) / d r$. In the outer parts of galaxies, these variables are linearly related; we therefore apply a linear fit and take the $y$-intercept-the magnitude at a null gradient—as our asymptotic magnitude.

\subsection{Classifying Disk Profiles}

Following the scheme laid out by previous work (see, e.g., Pohlen \& Trujillo 2006; E08, and references therein), we divide our profiles into three main broad categories: Type I (single exponential), Type II (down-bending), and Type III (up-bending). In Figure 2, we show individual examples of each of these types. Type I profiles require no further explanation; more details on Type II and III profiles are given below. 

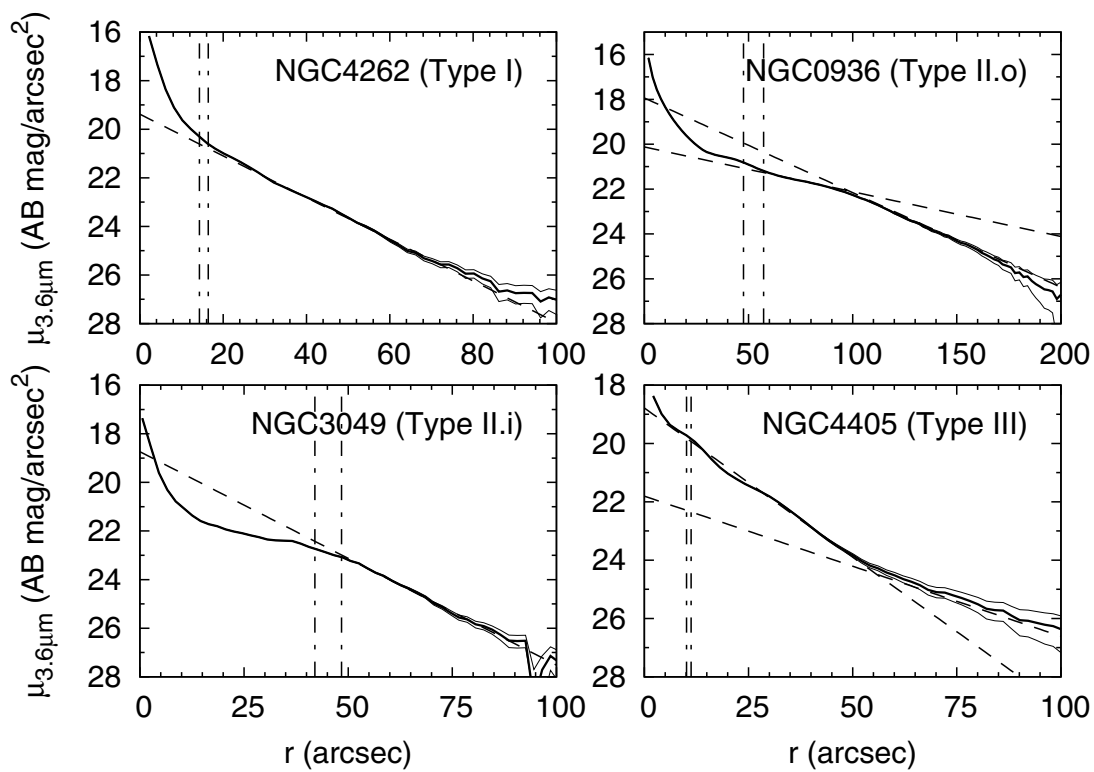

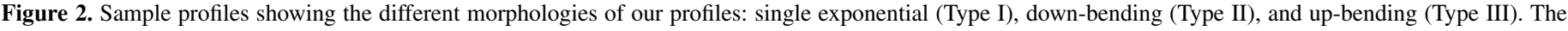

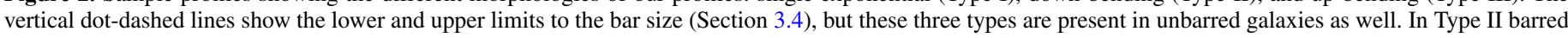
galaxies, the break can be at or inside the bar radius (Type II.i) or outside it (Type II.o). Dashed lines show fits to each component of the disk profile.

\subsubsection{Type II Profiles}

These profiles are characterized by a break beyond which the profile becomes steeper. In other words, the radial scale length of the outer disk is shorter than that of the inner one. In most cases, the break lies in the outer parts of the disk and, in particular, outside the radius of the bar, should there be one. Following E08, we refer to these profiles as Type II.o ("outer"). On the other hand, some barred galaxies exhibit a break which is so close to the bar radius that the profile actually looks purely exponential. However, when extrapolating this exponential toward the central regions, the result overpredicts the actual surface brightness of the bar and/or the bulge (Figure 2). This is in contrast to genuine Type I profiles, where the extrapolated exponential always lies below the bar and bulge components. To distinguish between these two cases, these "inner" breaks are denoted as Type II.i.

E08 went one step further and subdivided Type II.o profiles of barred galaxies into two categories, depending on their presumed physical origin. When the breaks were found between two or three times the bar radius, they referred to them as Type II.o-OLR, since they could be potentially linked to the OLR. If, however, the breaks were further out, they labeled them as Type II.o-CT profiles, since they seemed to be more similar to the "classical truncations" found in unbarred galaxies. While physically motivated, here we prefer to defer these interpretations to the Analysis section (in particular, Section 4.3), where a more quantitative comparison between the properties of breaks and bars will be presented.

\subsubsection{Type III Profiles}

Unlike Type II profiles, where the light distribution bends down beyond the break radius, Type III profiles exhibit a flatter slope outside the break. It should be noted, however, that the stellar haloes of early-type disks might contribute significantly to the light emitted in the outer parts of these galaxies, and these should be distinguished from the ones caused by a shallower scale length of the outer disk itself. Besides, Comerón et al. (2012) showed that the thick disk component with a flatter scale length than the thin disk can also lead to up-bending profiles.
In moderately inclined galaxies, the ellipticity profile is of great help here: if the ellipticity drops in the outer parts, the up-bending is most likely due to the spheroidal component, which is rounder than the disk seen in projection. Following E08, we denote those profiles as Type III-s ("spheroidal"). On the contrary, if the ellipticity remains roughly the same beyond the break radius, then we are probably witnessing a change of slope of the disk itself, and therefore name these profiles as Type III-d ("disk").

This method cannot be applied in galaxies close to face-on, where the ellipticity remains low throughout the whole profile. However, if we see structured emission in the outer parts of these galaxies, we consider these profiles to be Type III-d as well. Also, besides the ellipticity signature, the breaks in Type III-d profiles are usually sharper and better defined than in Type III-s ones, where the transition between the inner and outer slopes is smoother and more gradual.

A detailed analysis of Type III profiles is left for future papers but, for completeness, the galaxies in our sample exhibiting this kind of profile are also quoted in Table 2.

\subsection{Measuring the Properties of Disk Breaks}

In order to characterize the properties of our disk profiles, we follow a methodology similar to that established by previous authors in the field. As described before, for this particular purpose we employ the profiles measured with fixed ellipticity and P.A. and a $2^{\prime \prime}$ radial increment. The simplest case is that of a Type I profile, where we apply a linear fit to the disk-dominated region:

$$
I(r)=I_{0} e^{-r / h} \Rightarrow \mu(r)=\mu_{0}+1.086 \frac{r}{h},
$$

where $\mu_{0}$ is the central surface brightness of the disk and $h$ its exponential scale length. The inner boundary of the fitted region is set to exclude the bulge, as well as the shoulder of the bar, should there be one. The outer boundary is tuned according to the uncertainty in the surface brightness, to prevent spurious features and/or errors in the sky subtraction from biasing the 
Table 2

Profile Measurements

\begin{tabular}{|c|c|c|c|c|c|c|c|c|c|c|c|}
\hline Galaxy & $\begin{array}{c}\text { Prof. Type } \\
\text { (2) }\end{array}$ & $\begin{array}{l}r_{1, i} \\
\left({ }^{\prime \prime}\right) \\
(3)\end{array}$ & $\begin{array}{l}r_{2, i} \\
\left({ }^{\prime \prime}\right) \\
(4)\end{array}$ & $\begin{array}{l}r_{1, o} \\
\left({ }^{\prime \prime}\right) \\
(5)\end{array}$ & $\begin{array}{l}r_{2, o} \\
(") \\
(6)\end{array}$ & $\begin{array}{c}\mu_{0, i} \\
(\mathrm{mag} / \square) \\
\text { (7) }\end{array}$ & $\begin{array}{c}\mu_{0, o} \\
(\mathrm{mag} / \square) \\
(8)\end{array}$ & $\begin{array}{l}h_{i} \\
\left({ }^{\prime \prime}\right) \\
(9)\end{array}$ & $\begin{array}{l}h_{o} \\
\left({ }^{\prime \prime}\right) \\
(10)\end{array}$ & $\begin{array}{l}R_{\mathrm{br}} \\
\left({ }^{\prime \prime}\right) \\
(11)\end{array}$ & $\begin{array}{c}\alpha \\
\left(\prime^{\prime \prime}\right) \\
(12)\end{array}$ \\
\hline ESO026-001 & $2 \mathrm{i}+2 \mathrm{o}$ & 31.27 & 46.82 & 50.31 & 60.62 & $20.49_{-0.05}^{+0.09}$ & $18.77_{-0.56}^{+0.34}$ & $13.81_{-0.28}^{+0.45}$ & $9.38_{-0.90}^{+0.69}$ & $46.36_{-0.42}^{+0.65}$ & $6.29_{-2.03}^{+11.18}$ \\
\hline ESO027-001 & 20 & 46.34 & 63.28 & 70.20 & 99.94 & $20.82_{-0.25}^{+0.28}$ & $17.22_{-0.37}^{+0.58}$ & $32.68_{-3.65}^{+5.27}$ & $13.41_{-0.78}^{+1.44}$ & $75.20_{-1.33}^{+1.99}$ & $0.72_{-0.11}^{+2.88}$ \\
\hline ESO079-007 & $1+(2 o)$ & 17.37 & 57.30 & $\ldots$ & $\ldots$ & $20.88_{-0.10}^{+0.06}$ & $\ldots$ & $13.33_{-0.58}^{+0.44}$ & $\ldots$ & $\ldots$ & $\ldots$ \\
\hline ESO234-049 & 20 & 25.03 & 47.83 & 50.98 & 61.36 & $20.54_{-0.09}^{+0.14}$ & $18.37_{-0.41}^{+0.54}$ & $14.41_{-0.45}^{+0.58}$ & $8.99_{-0.61}^{+0.82}$ & $47.88_{-1.68}^{+0.29}$ & $5.08_{-1.90}^{+14.43}$ \\
\hline ESO482-035 & 20 & 27.19 & 55.48 & 59.02 & 72.28 & $20.58_{-0.05}^{+0.03}$ & $19.52_{-0.40}^{+0.77}$ & $17.18_{-0.33}^{+0.30}$ & $13.17_{-1.05}^{+2.63}$ & $55.20_{-13.40}^{+21.18}$ & $4.07_{-1.50}^{+10.05}$ \\
\hline ESO549-018 & 20 & 25.06 & 48.09 & 61.51 & 91.88 & $21.43_{-0.12}^{+0.06}$ & $18.63_{-0.44}^{+0.22}$ & $44.88_{-5.27}^{+4.14}$ & $13.76_{-1.08}^{+0.71}$ & $51.22_{-1.11}^{+2.07}$ & $0.37_{-0.07}^{+0.16}$ \\
\hline IC0101 & 1 & 14.86 & 45.65 & $\ldots$ & $\ldots$ & $20.09_{-0.10}^{+0.09}$ & $\ldots$ & $8.43_{-0.29}^{+0.28}$ & $\ldots$ & $\ldots$ & $\ldots$ \\
\hline IC0167 & 20 & 49.97 & 77.80 & $\cdots$ & $\ldots$ & $20.48_{-0.28}^{+0.20}$ & $\ldots$ & $16.72_{-1.23}^{+1.12}$ & $\ldots$ & $\ldots$ & $\ldots$ \\
\hline IC0749 & $2 \mathrm{i}+2 \mathrm{o}$ & 22.96 & 46.63 & 50.05 & 76.01 & $20.43_{-0.02}^{+0.05}$ & $18.61_{-0.24}^{+0.24}$ & $21.42_{-0.24}^{+0.45}$ & $12.34_{-0.64}^{+0.72}$ & $48.86_{-0.69}^{+0.58}$ & $3.24_{-2.05}^{+8.06}$ \\
\hline IC0797 & $3 d$ & 28.78 & 56.58 & 62.23 & 89.79 & $20.78_{-0.04}^{+0.04}$ & $22.11_{-0.17}^{+0.18}$ & $15.39_{-0.27}^{+0.28}$ & $23.23_{-1.55}^{+2.03}$ & $55.88_{-0.38}^{+0.44}$ & $3.87_{-1.74}^{+2.77}$ \\
\hline IC0800 & 1 & 35.83 & 77.63 & $\ldots$ & $\ldots$ & $20.78_{-0.15}^{+0.12}$ & $\ldots$ & $15.06_{-0.73}^{+0.70}$ & $\ldots$ & $\ldots$ & $\ldots$ \\
\hline IC1158 & 20 & 25.86 & 62.11 & 68.74 & 85.10 & $20.52_{-0.03}^{+0.05}$ & $17.56_{-0.45}^{+0.47}$ & $21.12_{-0.31}^{+0.44}$ & $11.22_{-0.74}^{+0.91}$ & $65.30_{-0.93}^{+0.66}$ & $0.75_{-0.23}^{+2.85}$ \\
\hline IC1265 & $3 d$ & 10.54 & 27.71 & 35.98 & 55.83 & $19.34_{-0.04}^{+0.06}$ & $21.51_{-0.15}^{+0.13}$ & $7.27_{-0.16}^{+0.20}$ & $16.83_{-1.28}^{+1.36}$ & $25.66_{-0.54}^{+0.56}$ & $0.60_{-0.14}^{+0.39}$ \\
\hline IC1826 & (1) & 25.03 & 63.96 & $\cdots$ & $\cdots$ & $20.72_{-0.13}^{+0.08}$ & $\ldots$ & $14.45_{-0.74}^{+0.58}$ & $\ldots$ & $\ldots$ & $\ldots$ \\
\hline IC1933 & 20 & 25.83 & 39.63 & 47.81 & 66.58 & $21.16_{-0.36}^{+0.19}$ & $16.99_{-0.37}^{+0.57}$ & $26.72_{-4.38}^{+3.95}$ & $8.93_{-0.43}^{+0.87}$ & $51.51_{-1.70}^{+1.42}$ & $0.61_{-0.14}^{+0.39}$ \\
\hline IC1954 & 1 & 18.98 & 125.40 & $\cdots$ & $\cdots$ & $19.32_{-0.06}^{+0.05}$ & $\ldots$ & $21.53_{-0.57}^{+0.50}$ & $\ldots$ & $\ldots$ & $\ldots$ \\
\hline IC2007 & $20+(3 d)$ & 16.47 & 23.66 & 26.50 & 45.22 & $19.86_{-0.18}^{+0.24}$ & $17.92_{-0.14}^{+0.16}$ & $12.46_{-1.07}^{+1.62}$ & $6.59_{-0.18}^{+0.24}$ & $25.00_{-0.46}^{+0.44}$ & $10.78_{-2.20}^{+7.79}$ \\
\hline IC2040 & $3 \mathrm{~s}$ & 15.41 & 39.52 & $\cdots$ & $\cdots$ & $19.70_{-0.08}^{+0.07}$ & & $8.59_{-0.20}^{+0.20}$ & $\ldots$ & $\ldots$ & $\ldots$ \\
\hline IC2051 & 20 & 45.88 & 52.94 & 54.90 & 102.74 & $20.77_{-0.45}^{+0.32}$ & $16.51_{-0.24}^{+0.18}$ & $146.20_{-150.55}^{+\cdots}$ & $12.89_{-0.51}^{+0.46}$ & $55.46_{-0.56}^{+0.94}$ & $2.55_{-1.23}^{+13.25}$ \\
\hline IC2056 & $3 d$ & 13.48 & 30.23 & 35.88 & 72.85 & $17.23_{-0.07}^{+0.05}$ & $19.63_{-0.11}^{+0.17}$ & $5.77_{-0.10}^{+0.07}$ & $11.27_{-0.34}^{+0.58}$ & $26.13_{-0.49}^{+0.67}$ & $1.02_{-0.23}^{+0.80}$ \\
\hline IC4237 & $2 \mathrm{i}$ & 20.53 & 70.16 & $\ldots$ & $\ldots$ & $18.93_{-0.05}^{+0.04}$ & $\ldots$ & $12.45_{-0.18}^{+0.18}$ & $\ldots$ & $\ldots$ & $\ldots$ \\
\hline IC5069 & 1 & 19.59 & 41.55 & $\ldots$ & $\ldots$ & $20.64_{-0.13}^{+0.11}$ & $\ldots$ & $9.04_{-0.43}^{+0.41}$ & $\ldots$ & $\ldots$ & $\ldots$ \\
\hline IC5332 & 20 & 81.45 & 170.44 & 190.97 & 236.15 & $22.21_{-0.12}^{+0.08}$ & $19.67_{-0.73}^{+0.51}$ & $88.92_{-4.46}^{+5.29}$ & $43.56_{-5.60}^{+6.29}$ & $199.64_{-4.76}^{+1.53}$ & $0.84_{-0.10}^{+0.63}$ \\
\hline NGC 0150 & $2 o+3 d$ & 27.81 & 42.51 & 48.39 & 65.24 & $19.58_{-0.02}^{+0.20}$ & $16.65_{-0.15}^{+0.15}$ & $40.86_{-0.05}^{+0.44}$ & $11.88_{-1.12}^{+1.27}$ & $45.16_{-0.32}^{+0.97}$ & $0.85_{-0.03}^{+0.09}$ \\
\hline NGC 0150 & $2 o+3 d$ & 50.53 & 64.71 & 83.69 & 135.29 & $16.50_{-0.02}^{+0.20}$ & $20.04_{-0.15}^{+0.15}$ & $11.53_{-0.05}^{+0.44}$ & $25.52_{-1.12}^{+1.27}$ & $68.64_{-0.32}^{+0.97}$ & $0.32_{-0.03}^{+0.09}$ \\
\hline NGC 0254 & 20 & 43.67 & 53.80 & 57.30 & 90.14 & $21.24_{-0.12}^{+0.09}$ & $19.93_{-0.17}^{+0.14}$ & $37.26_{-2.74}^{+2.71}$ & $20.62_{-1.15}^{+1.18}$ & $55.33_{-0.68}^{+0.90}$ & $1.43_{-0.50}^{+6.36}$ \\
\hline NGC 0255 & $3 d$ & 33.16 & 59.99 & 66.06 & 82.01 & $19.94_{-0.07}^{+0.05}$ & $22.07_{-0.20}^{+0.27}$ & $13.88_{-0.35}^{+0.30}$ & $24.74_{-1.88}^{+3.28}$ & $61.88_{-0.18}^{+0.59}$ & $3.69_{-1.12}^{+2.48}$ \\
\hline NGC 0289 & $1+(3 d)$ & 49.64 & 110.22 & $\cdots$ & $\cdots$ & $19.17_{-0.15}^{+0.12}$ & $\ldots$ & $19.96_{-0.81}^{+0.72}$ & $\ldots$ & $\ldots$ & $\ldots$ \\
\hline NGC 0300 & 1 & 112.30 & 626.38 & $\cdots$ & $\cdots$ & $21.11_{-0.06}^{+0.04}$ & $\ldots$ & $176.29_{-8.41}^{+7.54}$ & $\cdots$ & $\cdots$ & $\cdots$ \\
\hline NGC 0337 & $2 \mathrm{i}$ & 39.13 & 108.31 & $\cdots$ & $\ldots$ & $18.93_{-0.13}^{+0.12}$ & $\ldots$ & $15.67_{-0.52}^{+0.52}$ & $\cdots$ & $\cdots$ & $\ldots$ \\
\hline NGC 0406 & 1 & 35.81 & 104.25 & $\cdots$ & $\cdots$ & $20.65_{-0.11}^{+0.09}$ & $\cdots$ & $21.91_{-1.02}^{+0.91}$ & $\cdots$ & $\cdots$ & $\cdots$ \\
\hline NGC 0470 & $2 \mathrm{i}$ & 45.09 & 84.12 & $\ldots$ & $\ldots$ & $17.29_{-0.21}^{+0.20}$ & $\ldots$ & $12.13_{-0.52}^{+0.54}$ & $\ldots$ & $\ldots$ & $\ldots$ \\
\hline NGC 0473 & 1 & 29.18 & 64.32 & $\ldots$ & $\ldots$ & $19.43_{-0.14}^{+0.12}$ & $\ldots$ & $11.71_{-0.47}^{+0.44}$ & $\ldots$ & $\ldots$ & $\ldots$ \\
\hline NGC 0488 & $3(\mathrm{~d} ?)$ & 57.84 & 114.01 & 126.24 & 204.11 & $19.40_{-0.04}^{+0.03}$ & $19.92_{-0.20}^{+0.21}$ & $35.39_{-0.58}^{+0.54}$ & $41.05_{-2.49}^{+2.88}$ & $121.89_{-6.70}^{+8.27}$ & $0.41_{-0.15}^{+2.36}$ \\
\hline NGC 0628 & 1 & 64.88 & 371.83 & $\ldots$ & $\ldots$ & $20.23_{-0.05}^{+0.05}$ & $\ldots$ & $69.02_{-1.59}^{+1.68}$ & $\ldots$ & $\ldots$ & $\ldots$ \\
\hline NGC 0658 & $3 d$ & 24.57 & 41.54 & 66.33 & 83.94 & $19.80_{-0.27}^{+0.23}$ & $22.18_{-0.21}^{+0.25}$ & $13.02_{-1.04}^{+1.11}$ & $27.44_{-2.74}^{+4.42}$ & $54.30_{-3.49}^{+3.68}$ & $0.20_{-0.02}^{+0.06}$ \\
\hline NGC 0685 & 20 & 28.23 & 82.99 & 105.24 & 130.05 & $21.15_{-0.04}^{+0.03}$ & $18.88_{-0.67}^{+0.61}$ & $41.89_{-1.25}^{+1.30}$ & $22.01_{-2.26}^{+3.05}$ & $97.22_{-6.34}^{+4.19}$ & $0.23_{-0.04}^{+0.08}$ \\
\hline NGC 0691 & 20 & 37.76 & 85.03 & 98.04 & 113.91 & $20.27_{-0.15}^{+0.04}$ & $16.28_{-0.73}^{+0.91}$ & $27.64_{-1.33}^{+0.56}$ & $13.24_{-1.06}^{+1.78}$ & $93.17_{-3.27}^{+1.97}$ & $0.38_{-0.09}^{+0.21}$ \\
\hline NGC 0718 & 20 & 57.54 & 73.15 & 79.36 & 91.98 & $20.72_{-0.15}^{+0.09}$ & $17.37_{-0.64}^{+0.45}$ & $24.51_{-1.26}^{+0.92}$ & $12.30_{-1.05}^{+0.96}$ & $76.12_{-0.62}^{+0.93}$ & $0.81_{-0.27}^{+5.10}$ \\
\hline NGC 0723 & $2 o+3 d$ & 8.53 & 13.94 & 16.40 & 23.29 & $18.90_{-0.41}^{+0.66}$ & $17.32_{-0.17}^{+0.52}$ & $8.61_{-0.39}^{+0.82}$ & $4.38_{-0.57}^{+1.89}$ & $12.97_{-1.15}^{+3.06}$ & $30.79_{-0.08}^{+0.24}$ \\
\hline NGC 0723 & $2 o+3 d$ & 15.91 & 21.15 & 30.67 & 57.07 & $17.72_{-0.41}^{+0.66}$ & $21.16_{-0.17}^{+0.52}$ & $4.73_{-0.39}^{+0.82}$ & $11.26_{-0.57}^{+1.89}$ & $25.79_{-1.15}^{+3.06}$ & $0.53_{-0.08}^{+0.24}$ \\
\hline NGC 0772 & 20 & 55.54 & 114.13 & 122.26 & 175.44 & $19.84_{-0.03}^{+0.02}$ & $19.25_{-0.21}^{+0.22}$ & $46.61_{-0.76}^{+0.77}$ & $37.94_{-2.43}^{+3.17}$ & $110.79_{-6.96}^{+1.22}$ & $2.04_{-0.34}^{+3.12}$ \\
\hline NGC 0908 & $2 \mathrm{i}$ & 93.05 & 203.34 & $\ldots$ & $\ldots$ & $18.29_{-0.15}^{+0.13}$ & $\ldots$ & $30.16_{-1.04}^{+0.99}$ & $\ldots$ & $\ldots$ & $\ldots$ \\
\hline NGC 0918 & 20 & 27.25 & 66.73 & 72.67 & 122.98 & $20.17_{-0.06}^{+0.03}$ & $18.55_{-0.26}^{+0.18}$ & $34.30_{-1.15}^{+0.51}$ & $19.99_{-1.03}^{+0.87}$ & $71.85_{-1.28}^{+2.72}$ & $0.84_{-0.34}^{+5.69}$ \\
\hline NGC 0936 & 20 & 61.73 & 91.81 & 108.16 & 170.42 & $20.03_{-0.09}^{+0.07}$ & $18.02_{-0.21}^{+0.21}$ & $50.91_{-2.67}^{+2.69}$ & $25.86_{-1.12}^{+1.27}$ & $97.09_{-1.99}^{+2.24}$ & $0.31_{-0.08}^{+0.30}$ \\
\hline NGC 0941 & 20 & 38.93 & 76.33 & 80.25 & 85.68 & $21.20_{-0.10}^{+0.05}$ & $13.91_{-\ldots}^{+2.73}$ & $22.20_{-0.71}^{+0.75}$ & $7.72_{-4.20}^{+3.10}$ & $79.57_{-1.77}^{+5.45}$ & $2.58_{-1.04}^{+7.17}$ \\
\hline NGC 0986 & $2 \mathrm{i}$ & 61.09 & 142.54 & $\ldots$ & $\ldots$ & $18.69_{-0.13}^{+0.12}$ & $\ldots$ & $22.16_{-0.66}^{+0.68}$ & $\ldots$ & $\ldots$ & $\ldots$ \\
\hline NGC 0991 & 20 & 37.61 & 63.64 & 65.42 & 82.00 & $21.05_{-0.09}^{+0.02}$ & $18.85_{-0.35}^{+0.34}$ & $23.92_{-0.81}^{+0.48}$ & $13.70_{-0.82}^{+1.09}$ & $64.89_{-0.70}^{+0.78}$ & $5.62_{-3.25}^{+19.75}$ \\
\hline NGC 1015 & 1 & 31.17 & 89.74 & $\cdots$ & $\ldots$ & $20.55_{-0.12}^{+0.08}$ & $\ldots$ & $18.70_{-0.82}^{+0.69}$ & $\ldots$ & $\ldots$ & $\ldots$ \\
\hline NGC 1022 & 1 & 36.19 & 92.66 & $\ldots$ & $\ldots$ & $19.96_{-0.08}^{+0.07}$ & $\ldots$ & $20.25_{-0.72}^{+0.72}$ & $\ldots$ & $\ldots$ & $\ldots$ \\
\hline NGC 1042 & 20 & 47.99 & 78.78 & 91.46 & 161.18 & $21.22_{-0.08}^{+0.04}$ & $19.66_{-0.20}^{+0.16}$ & $56.45_{-3.01}^{+1.64}$ & $29.85_{-1.57}^{+1.55}$ & $90.53_{-1.24}^{+2.17}$ & $1.29_{-0.72}^{+1.26}$ \\
\hline NGC 1073 & 20 & 74.85 & 108.72 & 121.92 & 150.03 & $21.45_{-0.04}^{+0.07}$ & $18.13_{-0.63}^{+0.49}$ & $50.79_{-1.41}^{+2.31}$ & $22.47_{-2.10}^{+2.21}$ & $123.09_{-1.87}^{+1.18}$ & $0.38_{-0.11}^{+1.24}$ \\
\hline
\end{tabular}


Table 2

(Continued)

\begin{tabular}{|c|c|c|c|c|c|c|c|c|c|c|c|}
\hline Galaxy & $\begin{array}{c}\text { Prof. Type } \\
\text { (2) }\end{array}$ & $\begin{array}{l}r_{1, i} \\
\left({ }^{\prime \prime}\right) \\
(3)\end{array}$ & $\begin{array}{l}r_{2, i} \\
(") \\
(4)\end{array}$ & $\begin{array}{l}r_{1, o} \\
(") \\
(5)\end{array}$ & $\begin{array}{l}r_{2, o} \\
(") \\
(6)\end{array}$ & $\begin{array}{c}\mu_{0, i} \\
(\mathrm{mag} / \square) \\
\text { (7) }\end{array}$ & $\begin{array}{c}\mu_{0, o} \\
(\mathrm{mag} / \square) \\
(8)\end{array}$ & $\begin{array}{l}h_{i} \\
(") \\
(9)\end{array}$ & $\begin{array}{c}h_{o} \\
(") \\
(10)\end{array}$ & $\begin{array}{l}R_{\mathrm{br}} \\
\left({ }^{\prime \prime}\right) \\
(11)\end{array}$ & $\begin{array}{c}\alpha \\
\left(I^{\prime \prime}\right) \\
(12)\end{array}$ \\
\hline NGC 1084 & $3 d$ & 53.03 & 83.10 & 86.27 & 108.43 & $18.39_{-0.08}^{+0.11}$ & $20.17_{-0.25}^{+0.30}$ & $16.76_{-0.39}^{+0.49}$ & $24.93_{-1.87}^{+2.73}$ & $83.78_{-0.67}^{+1.72}$ & $1.58_{-0.82}^{+12.89}$ \\
\hline NGC 1087 & 20 & 29.85 & 50.28 & 57.42 & 119.71 & $20.01_{-0.24}^{+0.25}$ & $18.57_{-0.14}^{+0.12}$ & $41.80_{-6.94}^{+15.39}$ & $19.86_{-0.70}^{+0.76}$ & $49.95_{-2.15}^{+2.54}$ & $0.70_{-0.21}^{+4.26}$ \\
\hline NGC 1097 & 20 & 144.16 & 214.26 & 234.10 & 353.14 & $21.82_{-0.11}^{+0.03}$ & $18.86_{-0.22}^{+0.22}$ & $201.58_{-17.46}^{+7.78}$ & $58.25_{-2.64}^{+3.23}$ & $223.15_{-1.38}^{+2.72}$ & $0.25_{-0.08}^{+1.27}$ \\
\hline NGC 1179 & $2 \mathrm{i}$ & 34.79 & 151.73 & $\ldots$ & $\ldots$ & $21.62_{-0.07}^{+0.05}$ & $\ldots$ & $39.09_{-1.66}^{+1.41}$ & $\ldots$ & $\ldots$ & $\ldots$ \\
\hline NGC 1187 & $2 \mathrm{i}$ & 35.42 & 109.71 & $\ldots$ & $\ldots$ & $19.69_{-0.02}^{+0.02}$ & $\ldots$ & $30.09_{-0.36}^{+0.25}$ & $\ldots$ & $\ldots$ & $\ldots$ \\
\hline NGC 1222 & $3 d$ & 17.18 & 39.43 & 50.41 & 95.76 & $19.81_{-0.25}^{+0.14}$ & $22.13_{-0.14}^{+0.10}$ & $11.69_{-0.94}^{+0.63}$ & $28.23_{-2.06}^{+2.02}$ & $42.69_{-2.12}^{+1.20}$ & $0.46_{-0.10}^{+0.27}$ \\
\hline NGC 1232 & 20 & 49.71 & 183.27 & 206.63 & 242.56 & $20.15_{-0.03}^{+0.05}$ & $17.40_{-0.50}^{+0.79}$ & $50.05_{-0.72}^{+0.78}$ & $30.37_{-2.12}^{+4.18}$ & $195.44_{-4.29}^{+2.28}$ & $0.21_{-0.05}^{+0.47}$ \\
\hline NGC 1255 & 20 & 38.50 & 58.56 & 74.60 & 125.13 & $20.86_{-0.09}^{+0.03}$ & $18.97_{-0.18}^{+0.16}$ & $54.08_{-3.98}^{+1.39}$ & $21.31_{-1.05}^{+1.05}$ & $61.13_{-0.82}^{+0.67}$ & $6.01_{-1.27}^{+2.57}$ \\
\hline NGC 1258 & 20 & 14.79 & 27.27 & 29.77 & 47.41 & $20.74_{-0.08}^{+0.02}$ & $19.37_{-0.14}^{+0.12}$ & $15.39_{-0.69}^{+0.21}$ & $9.00_{-0.34}^{+0.32}$ & $27.28_{-0.27}^{+0.55}$ & $2.00_{-0.58}^{+12.21}$ \\
\hline NGC 1299 & $1+(3 s)$ & 17.61 & 46.20 & $\cdots$ & $\cdots$ & $18.56_{-0.07}^{+0.07}$ & $\ldots$ & $7.54_{-0.14}^{+0.15}$ & $\ldots$ & $\ldots$ & $\ldots$ \\
\hline NGC 1300 & 20 & 128.34 & 173.35 & 185.83 & 220.59 & $21.49_{-0.16}^{+0.06}$ & $16.23_{-1.16}^{+0.72}$ & $69.64_{-4.59}^{+2.47}$ & $24.32_{-2.82}^{+2.53}$ & $180.87_{-1.38}^{+2.60}$ & $0.40_{-0.15}^{+2.42}$ \\
\hline NGC 1302 & 20 & 59.84 & 98.27 & 104.31 & 158.66 & $21.25_{-0.05}^{+0.07}$ & $18.89_{-0.28}^{+0.22}$ & $54.39_{-1.31}^{+2.38}$ & $24.77_{-1.39}^{+1.26}$ & $98.77_{-1.34}^{+1.01}$ & $2.68_{-0.32}^{+4.35}$ \\
\hline NGC 1306 & $3 d$ & 8.21 & 31.19 & 33.11 & 40.17 & $18.46_{-0.03}^{+0.03}$ & $21.53_{-0.62}^{+0.30}$ & $5.41_{-0.07}^{+0.05}$ & $10.29_{-1.50}^{+1.14}$ & $32.22_{-0.56}^{+0.42}$ & $5.21_{-1.02}^{+18.20}$ \\
\hline NGC $1325 \mathrm{~A}$ & $2 \mathrm{i}+(2 \mathrm{o})$ & 26.67 & 80.00 & $\ldots$ & $\ldots$ & $20.87_{-0.15}^{+0.10}$ & $\ldots$ & $17.13_{-1.06}^{+0.88}$ & $\ldots$ & $\ldots$ & $\ldots$ \\
\hline NGC 1326 & 20 & 56.47 & 73.80 & 86.63 & 165.88 & $21.45_{-0.27}^{+0.25}$ & $19.80_{-0.10}^{+0.08}$ & $91.92_{-21.48}^{+51.53}$ & $31.30_{-0.96}^{+0.94}$ & $71.91_{-1.00}^{+1.55}$ & $2.41_{-1.06}^{+3.36}$ \\
\hline NGC 1338 & $2 \mathrm{i}$ & 19.02 & 42.07 & $\ldots$ & $\ldots$ & $19.10_{-0.10}^{+0.12}$ & $\ldots$ & $9.31_{-0.26}^{+0.37}$ & $\ldots$ & $\ldots$ & $\ldots$ \\
\hline NGC 1341 & 3 & 26.63 & 41.87 & 52.14 & 73.96 & $19.05_{-0.30}^{+0.15}$ & $21.64_{-0.12}^{+0.07}$ & $8.87_{-0.58}^{+0.33}$ & $17.13_{-0.58}^{+0.39}$ & $43.73_{-1.55}^{+0.82}$ & $0.49_{-0.09}^{+0.18}$ \\
\hline NGC 1347 & $20+(3 d)$ & 14.62 & 30.84 & 33.51 & 52.41 & $21.15_{-0.01}^{+0.03}$ & $19.06_{-0.29}^{+0.26}$ & $15.53_{-0.15}^{+0.28}$ & $8.05_{-0.46}^{+0.48}$ & $32.19_{-0.54}^{+0.44}$ & $1.87_{-0.59}^{+13.09}$ \\
\hline NGC 1350 & 20 & 119.78 & 154.52 & 162.31 & 238.37 & $22.02_{-0.13}^{+0.06}$ & $18.98_{-0.32}^{+0.24}$ & $159.04_{-19.60}^{+13.25}$ & $40.44_{-2.66}^{+2.43}$ & $152.07_{-1.78}^{+1.87}$ & $1.22_{-0.32}^{+3.83}$ \\
\hline NGC 1357 & 20 & 60.43 & 90.37 & 98.13 & 134.76 & $20.82_{-0.11}^{+0.06}$ & $19.65_{-0.43}^{+0.34}$ & $38.68_{-1.89}^{+1.70}$ & $27.19_{-2.85}^{+3.14}$ & $98.25_{-2.33}^{+2.07}$ & $0.64_{-0.24}^{+4.14}$ \\
\hline NGC 1367 & $3 d$ & 52.23 & 95.85 & 127.42 & 216.39 & $19.64_{-0.10}^{+0.07}$ & $20.43_{-0.25}^{+0.14}$ & $31.54_{-1.41}^{+0.82}$ & $42.42_{-2.76}^{+2.09}$ & $90.03_{-13.79}^{+4.96}$ & $0.42_{-0.17}^{+2.26}$ \\
\hline NGC 1385 & 1 & 71.92 & 111.76 & $\cdots$ & $\cdots$ & $19.65_{-0.22}^{+0.19}$ & $\ldots$ & $23.96_{-1.46}^{+1.45}$ & $\ldots$ & $\ldots$ & $\ldots$ \\
\hline NGC 1398 & $2 o+3 d$ & 82.54 & 165.09 & 182.08 & 229.83 & $20.48_{-0.33}^{+0.39}$ & $18.38_{-0.60}^{+0.39}$ & $69.47_{-2.28}^{+3.09}$ & $39.68_{-11.69}^{+12.36}$ & $178.93_{-2.21}^{+3.41}$ & $0.29_{-0.53}^{+2.53}$ \\
\hline NGC 1398 & $2 o+3 d$ & 178.04 & 222.55 & 233.88 & 326.94 & $18.38_{-0.33}^{+0.39}$ & $21.36_{-0.60}^{+0.39}$ & $39.69_{-2.28}^{+3.09}$ & $75.51_{-11.69}^{+12.36}$ & $229.33_{-2.21}^{+3.41}$ & $0.97_{-0.53}^{+2.53}$ \\
\hline NGC 1433 & 20 & 135.06 & 173.06 & 188.26 & 224.51 & $21.34_{-0.10}^{+0.12}$ & $17.32_{-0.54}^{+0.52}$ & $83.32_{-3.88}^{+5.63}$ & $31.29_{-2.36}^{+2.87}$ & $185.50_{-2.04}^{+1.21}$ & $0.33_{-0.11}^{+1.97}$ \\
\hline NGC 1436 & 20 & 27.70 & 44.92 & 49.66 & 111.05 & $21.15_{-0.15}^{+0.03}$ & $19.38_{-0.09}^{+0.09}$ & $124.60_{-35.68}^{+10.51}$ & $20.82_{-0.66}^{+0.68}$ & $40.76_{-0.57}^{+0.82}$ & $8.82_{-4.29}^{+13.91}$ \\
\hline NGC 1438 & $2 \mathrm{i}$ & 35.94 & 66.13 & $\cdots$ & $\cdots$ & $17.59_{-0.12}^{+0.14}$ & $\ldots$ & $9.86_{-0.22}^{+0.28}$ & $\ldots$ & $\ldots$ & $\ldots$ \\
\hline NGC 1452 & 20 & 61.84 & 74.86 & 79.89 & 97.94 & $19.99_{-0.21}^{+0.15}$ & $15.46_{-0.62}^{+0.52}$ & $23.98_{-1.62}^{+1.29}$ & $10.40_{-0.71}^{+0.73}$ & $76.68_{-0.73}^{+0.72}$ & $0.99_{-0.36}^{+8.17}$ \\
\hline NGC 1493 & $2 \mathrm{i}$ & 38.77 & 122.19 & $\ldots$ & $\ldots$ & $20.17_{-0.07}^{+0.07}$ & $\ldots$ & $24.51_{-0.68}^{+0.72}$ & $\ldots$ & $\ldots$ & $\ldots$ \\
\hline NGC 1494 & 20 & 41.98 & 74.87 & 80.21 & 119.79 & $21.23_{-0.08}^{+0.02}$ & $19.76_{-0.23}^{+0.25}$ & $35.87_{-1.48}^{+0.59}$ & $22.60_{-1.30}^{+1.64}$ & $82.66_{-1.13}^{+2.14}$ & $1.19_{-0.39}^{+4.11}$ \\
\hline NGC 1512 & 20 & 134.22 & 193.05 & 201.60 & 250.27 & $22.14_{-0.29}^{+0.08}$ & $19.85_{-0.45}^{+0.31}$ & $88.53_{-11.84}^{+5.26}$ & $43.44_{-3.82}^{+3.36}$ & $179.87_{-2.28}^{+6.22}$ & $0.58_{-0.26}^{+4.05}$ \\
\hline NGC 1533 & $2 \mathrm{i}$ & 47.33 & 105.55 & $\cdots$ & $\cdots$ & $18.84_{-0.13}^{+0.14}$ & $\ldots$ & $19.09_{-0.78}^{+0.91}$ & $\ldots$ & $\ldots$ & $\ldots$ \\
\hline NGC 1559 & $2 i+3 d$ & 37.88 & 95.93 & 106.27 & 158.91 & $17.57_{-0.18}^{+0.16}$ & $18.95_{-0.31}^{+0.29}$ & $17.78_{-0.66}^{+0.35}$ & $23.26_{-1.33}^{+1.35}$ & $96.03_{-4.19}^{+2.41}$ & $1.77_{-0.94}^{+2.63}$ \\
\hline NGC 1566 & $3 \mathrm{~d}$ & 60.83 & 174.05 & $\ldots$ & $\ldots$ & $19.43_{-0.06}^{+0.04}$ & $\ldots$ & $40.23_{-0.83}^{+0.78}$ & $\ldots$ & $\ldots$ & $\ldots$ \\
\hline NGC 1637 & $2 o+3 d$ & 44.17 & 60.27 & 77.11 & 138.88 & $21.02_{-0.11}^{+0.15}$ & $17.97_{-0.27}^{+0.16}$ & $126.59_{-0.46}^{+0.65}$ & $20.84_{-5.42}^{+5.43}$ & $69.98_{-1.11}^{+1.10}$ & $0.30_{-0.26}^{+5.70}$ \\
\hline NGC 1637 & $2 o+3 d$ & 86.47 & 134.76 & 142.24 & 177.06 & $17.93_{-0.11}^{+0.15}$ & $22.45_{-0.27}^{+0.16}$ & $20.66_{-0.46}^{+0.65}$ & $55.99_{-5.42}^{+5.43}$ & $136.17_{-1.11}^{+1.10}$ & $0.67_{-0.26}^{+5.70}$ \\
\hline NGC 1640 & 1 & 40.47 & 94.12 & $\cdots$ & $\cdots$ & $20.14_{-0.19}^{+0.12}$ & $\ldots$ & $20.32_{-1.30}^{+1.07}$ & $\ldots$ & $\ldots$ & $\ldots$ \\
\hline NGC 1672 & 20 & 91.98 & 115.15 & 129.41 & 187.88 & $21.64_{-0.53}^{+0.43}$ & $18.73_{-0.23}^{+0.20}$ & $206.18_{-92.86}^{+\cdots}$ & $36.82_{-2.06}^{+2.28}$ & $120.46_{-2.22}^{+3.59}$ & $0.35_{-0.09}^{+1.44}$ \\
\hline NGC 1688 & 20 & 42.14 & 67.59 & 71.66 & 106.31 & $20.27_{-0.10}^{+0.07}$ & $18.63_{-0.51}^{+0.33}$ & $22.89_{-0.88}^{+0.52}$ & $15.55_{-1.35}^{+1.11}$ & $73.24_{-1.14}^{+2.54}$ & $1.23_{-0.51}^{+6.87}$ \\
\hline NGC 1703 & 20 & 28.27 & 70.46 & 73.72 & 100.90 & $20.00_{-0.04}^{+0.02}$ & $18.95_{-0.61}^{+0.44}$ & $20.79_{-0.34}^{+0.28}$ & $15.99_{-1.74}^{+1.90}$ & $66.75_{-14.51}^{+2.73}$ & $3.07_{-0.52}^{+6.78}$ \\
\hline NGC 1792 & $2 i+3 d$ & 64.45 & 109.76 & 127.63 & 171.66 & $17.69_{-0.09}^{+0.03}$ & $18.40_{-0.35}^{+0.22}$ & $24.61_{-0.52}^{+0.19}$ & $28.85_{-1.97}^{+1.65}$ & $109.55_{-20.50}^{+1.72}$ & $2.92_{-1.52}^{+3.58}$ \\
\hline NGC 1808 & $2 \mathrm{i}$ & 196.52 & 303.21 & $\ldots$ & $\cdots$ & $19.71_{-0.32}^{+0.32}$ & $\ldots$ & $51.31_{-3.47}^{+4.27}$ & $\ldots$ & $\ldots$ & $\ldots$ \\
\hline NGC 2500 & 20 & 39.89 & 62.58 & 69.51 & 87.42 & $21.19_{-0.12}^{+0.09}$ & $18.48_{-0.67}^{+0.39}$ & $41.07_{-2.84}^{+2.97}$ & $15.74_{-1.91}^{+1.59}$ & $63.73_{-0.99}^{+1.20}$ & $2.34_{-1.10}^{+6.47}$ \\
\hline NGC 2633 & $2 \mathrm{i}+2 \mathrm{o}$ & 39.27 & 58.78 & 65.20 & 83.94 & $19.72_{-0.07}^{+0.10}$ & $18.00_{-0.24}^{+0.20}$ & $17.22_{-0.52}^{+0.57}$ & $11.56_{-0.48}^{+0.45}$ & $55.93_{-0.32}^{+0.45}$ & $6.03_{-0.92}^{+2.55}$ \\
\hline NGC 2805 & 20 & 43.92 & 119.39 & 131.36 & 156.92 & $22.02_{-0.16}^{+0.07}$ & $18.11_{-1.78}^{+0.67}$ & $54.13_{-4.80}^{+2.31}$ & $21.66_{-4.22}^{+2.90}$ & $130.10_{-0.42}^{+4.39}$ & $1.07_{-0.06}^{+1.00}$ \\
\hline NGC 2841 & 1 & 128.91 & 301.75 & $\cdots$ & $\cdots$ & $18.75_{-0.14}^{+0.12}$ & $\ldots$ & $49.99_{-1.92}^{+1.88}$ & $\ldots$ & $\ldots$ & $\ldots$ \\
\hline NGC 2844 & 1 & 34.22 & 73.47 & $\ldots$ & $\ldots$ & $20.96_{-0.13}^{+0.11}$ & $\cdots$ & $16.82_{-0.87}^{+0.89}$ & $\cdots$ & $\ldots$ & $\cdots$ \\
\hline NGC 2906 & $2 \mathrm{i}$ & 21.97 & 71.66 & $\ldots$ & $\ldots$ & $17.89_{-0.09}^{+0.08}$ & $\ldots$ & $9.39_{-0.19}^{+0.19}$ & $\ldots$ & $\ldots$ & $\ldots$ \\
\hline NGC 2964 & 1 & 47.51 & 105.16 & $\ldots$ & $\cdots$ & $18.74_{-0.08}^{+0.07}$ & $\cdots$ & $17.15_{-0.35}^{+0.36}$ & $\cdots$ & $\cdots$ & $\cdots$ \\
\hline NGC 3032 & 1 & 32.11 & 70.31 & $\ldots$ & $\ldots$ & $20.62_{-0.14}^{+0.11}$ & $\cdots$ & $16.79_{-0.96}^{+0.92}$ & $\cdots$ & $\cdots$ & $\cdots$ \\
\hline NGC 3049 & $2 \mathrm{i}$ & 51.21 & 84.91 & $\cdots$ & $\cdots$ & $18.74_{-0.28}^{+0.24}$ & $\cdots$ & $12.37_{-0.66}^{+0.66}$ & $\cdots$ & $\cdots$ & $\cdots$ \\
\hline NGC 3073 & 1 & 41.65 & 78.67 & $\ldots$ & $\ldots$ & $22.63_{-0.14}^{+0.12}$ & $\ldots$ & $24.36_{-1.93}^{+2.14}$ & $\cdots$ & $\ldots$ & $\ldots$ \\
\hline
\end{tabular}


Table 2

(Continued)

\begin{tabular}{|c|c|c|c|c|c|c|c|c|c|c|c|}
\hline Galaxy & (2) & $\begin{array}{l}r_{1, i} \\
\left({ }^{\prime \prime}\right) \\
(3)\end{array}$ & $\begin{array}{l}r_{2, i} \\
\left({ }^{\prime \prime}\right) \\
(4)\end{array}$ & $\begin{array}{l}r_{1, o} \\
\left({ }^{\prime \prime}\right) \\
(5)\end{array}$ & $\begin{array}{l}r_{2, o} \\
(") \\
(6)\end{array}$ & $\begin{array}{c}\mu_{0, i} \\
(\mathrm{mag} / \square) \\
(7)\end{array}$ & $\begin{array}{c}\mu_{0, o} \\
(\mathrm{mag} / \square) \\
(8)\end{array}$ & $\begin{array}{l}h_{i} \\
(") \\
(9)\end{array}$ & $\begin{array}{l}h_{o} \\
\left({ }^{\prime \prime}\right) \\
(10)\end{array}$ & $\begin{array}{l}R_{\mathrm{br}} \\
\left({ }^{\prime \prime}\right) \\
(11)\end{array}$ & $\begin{array}{c}\alpha \\
\left(l^{\prime \prime}\right) \\
(12)\end{array}$ \\
\hline NGC 3147 & 1 & 51.36 & 143.59 & $\cdots$ & $\ldots$ & $19.12_{-0.12}^{+0.09}$ & $\ldots$ & $25.29_{-0.95}^{+0.85}$ & $\cdots$ & $\ldots$ & $\ldots$ \\
\hline NGC 3184 & 20 & 41.07 & 114.08 & 127.77 & 221.77 & $21.04_{-0.05}^{+0.01}$ & $19.31_{-0.12}^{+0.16}$ & $120.97_{-9.65}^{+1.33}$ & $43.50_{-1.50}^{+2.54}$ & $108.30_{-1.61}^{+2.42}$ & $0.64_{-0.27}^{+1.76}$ \\
\hline NGC 3206 & 20 & 34.53 & 58.16 & 63.72 & 79.95 & $21.68_{-0.07}^{+0.07}$ & $18.75_{-0.40}^{+0.33}$ & $29.25_{-1.67}^{+1.22}$ & $13.10_{-1.00}^{+0.98}$ & $64.12_{-0.19}^{+0.92}$ & $3.18_{-0.12}^{+3.63}$ \\
\hline NGC 3319 & 20 & 79.51 & 159.44 & 170.37 & 188.88 & $22.42_{-0.06}^{+0.32}$ & $17.20_{-2.10}^{+2.74}$ & $76.89_{-3.83}^{+25.98}$ & $23.51_{-4.55}^{+14.16}$ & $162.61_{-23.93}^{+4.65}$ & $0.46_{-0.16}^{+3.64}$ \\
\hline NGC 3344 & 20 & 38.50 & 87.34 & 97.33 & 166.84 & $20.12_{-0.09}^{+0.05}$ & $18.84_{-0.12}^{+0.11}$ & $50.63_{-1.92}^{+1.80}$ & $30.75_{-0.86}^{+0.98}$ & $92.19_{-1.55}^{+2.32}$ & $1.81_{-0.98}^{+2.89}$ \\
\hline NGC 3351 & 20 & 116.58 & 144.38 & 157.75 & 249.73 & $20.58_{-0.35}^{+0.12}$ & $18.72_{-0.18}^{+0.14}$ & $82.24_{-12.90}^{+6.75}$ & $41.34_{-1.69}^{+1.54}$ & $142.06_{-1.98}^{+4.22}$ & $0.37_{-0.12}^{+2.99}$ \\
\hline NGC 3368 & 20 & 148.73 & 171.04 & 185.92 & 362.27 & $21.57_{-0.23}^{+0.18}$ & $19.70_{-0.08}^{+0.07}$ & $181.76_{-40.43}^{+167.76}$ & $64.00_{-1.43}^{+1.32}$ & $170.02_{-1.33}^{+2.44}$ & $0.94_{-0.41}^{+2.86}$ \\
\hline NGC 3486 & 20 & 86.45 & 164.88 & 184.49 & 228.61 & $21.71_{-0.18}^{+0.06}$ & $15.35_{-1.67}^{+1.56}$ & $61.97_{-3.21}^{+1.65}$ & $21.87_{-2.89}^{+4.43}$ & $\begin{array}{l}198.05_{-4.05}^{+3.35} \\
0\end{array}$ & $0.26_{-0.05}^{+0.56}$ \\
\hline NGC 3504 & 1 & 41.34 & 116.08 & $\ldots$ & $\ldots$ & $19.97_{-0.15}^{+0.08}$ & $\ldots$ & $20.88_{-0.86}^{+0.62}$ & $\ldots$ & $\ldots$ & $\ldots$ \\
\hline NGC 3627 & $2 i+3 d$ & 111.68 & 169.41 & 257.25 & 406.59 & $17.81_{-0.08}^{+0.12}$ & $20.49_{-0.21}^{+0.20}$ & $43.26_{-1.02}^{+1.52}$ & $84.98_{-5.22}^{+5.86}$ & $217.83_{-4.98}^{+5.66}$ & $0.06_{-0.01}^{+0.01}$ \\
\hline NGC 3726 & $2 \mathrm{i}+2 \mathrm{o}$ & 60.96 & 130.46 & 153.62 & 203.00 & $19.94_{-0.05}^{+0.07}$ & $16.15_{-0.56}^{+0.70}$ & $47.57_{-0.98}^{+1.37}$ & $23.06_{-1.47}^{+2.40}$ & $156.29_{-4.46}^{+2.39}$ & $0.22_{-0.05}^{+0.12}$ \\
\hline NGC 3794 & 1 & 19.23 & 91.53 & $\ldots$ & $\ldots$ & $20.93_{-0.05}^{+0.05}$ & $\ldots$ & $18.48_{-0.55}^{+0.53}$ & $\ldots$ & $\ldots$ & $\ldots$ \\
\hline NGC 3938 & 20 & 46.42 & 151.19 & 158.26 & 177.71 & $19.84_{-0.06}^{+0.01}$ & $17.24_{-1.37}^{+0.86}$ & $33.48_{-0.48}^{+0.43}$ & $21.73_{-3.11}^{+2.57}$ & $148.15_{-6.45}^{+3.99}$ & $1.41_{-0.39}^{+4.30}$ \\
\hline NGC 3953 & 20 & 73.81 & 158.36 & 166.96 & 193.47 & $19.25_{-0.08}^{+0.02}$ & $14.86_{-0.78}^{+0.69}$ & $45.80_{-0.95}^{+0.83}$ & $21.47_{-1.78}^{+2.16}$ & $163.30_{-1.20}^{+1.21}$ & $1.16_{-0.76}^{+5.55}$ \\
\hline NGC 4051 & 20 & 105.68 & 138.04 & 145.37 & 191.10 & $21.21_{-0.22}^{+0.02}$ & $18.21_{-0.46}^{+0.30}$ & $71.26_{-6.42}^{+1.47}$ & $29.73_{-2.31}^{+1.96}$ & $140.73_{-0.77}^{+1.76}$ & $1.28_{-0.54}^{+4.29}$ \\
\hline NGC 4136 & 20 & 37.42 & 90.62 & 93.84 & 111.96 & $20.83_{-0.09}^{+0.06}$ & $18.79_{-0.38}^{+0.51}$ & $28.12_{-0.64}^{+0.66}$ & $17.74_{-1.18}^{+1.95}$ & $90.30_{-1.59}^{+0.91}$ & $3.11_{-1.94}^{+12.53}$ \\
\hline NGC 4245 & 20 & 60.42 & 74.51 & 78.84 & 111.09 & $20.89_{-0.09}^{+0.09}$ & $19.22_{-0.33}^{+0.30}$ & $33.20_{-1.51}^{+1.80}$ & $20.27_{-1.49}^{+1.69}$ & $79.93_{-1.57}^{+1.09}$ & $1.16_{-0.51}^{+7.51}$ \\
\hline NGC 4254 & 20 & 56.47 & 154.01 & 163.63 & 192.51 & $19.22_{-0.08}^{+0.01}$ & $14.90_{-1.16}^{+0.60}$ & $39.62_{-0.48}^{+0.27}$ & $19.52_{-2.09}^{+1.51}$ & $153.27_{-1.97}^{+2.74}$ & $1.04_{-0.36}^{+2.82}$ \\
\hline NGC 4262 & 1 & 22.40 & 77.85 & $\ldots$ & $\ldots$ & $19.36_{-0.09}^{+0.09}$ & $\ldots$ & $12.66_{-0.37}^{+0.40}$ & $\ldots$ & $\ldots$ & $\ldots$ \\
\hline NGC 4298 & $2 o+3 d$ & 37.42 & 58.76 & 64.90 & 87.99 & $19.35_{-0.09}^{+0.06}$ & $18.30_{-0.09}^{+0.08}$ & $27.36_{-0.43}^{+0.31}$ & $19.13_{-1.17}^{+1.14}$ & $61.11_{-0.76}^{+0.42}$ & $0.81_{-0.06}^{+0.14}$ \\
\hline NGC 4298 & $2 o+3 d$ & 64.90 & 88.28 & 108.16 & 159.61 & $18.37_{-0.09}^{+0.06}$ & $21.11_{-0.09}^{+0.08}$ & $19.45_{-0.43}^{+0.31}$ & $38.47_{-1.17}^{+1.14}$ & $99.27_{-0.76}^{+0.42}$ & $0.25_{-0.06}^{+0.14}$ \\
\hline NGC 4303 & 20 & 94.47 & 152.85 & 159.98 & 204.99 & $19.55_{-0.21}^{+0.05}$ & $18.45_{-0.53}^{+0.54}$ & $40.84_{-1.54}^{+0.78}$ & $32.19_{-2.71}^{+4.01}$ & $154.02_{-35.57}^{+6.82}$ & $1.91_{-0.65}^{+3.47}$ \\
\hline NGC 4314 & $20+(3)$ & 84.53 & 113.49 & 121.89 & 156.45 & $20.70_{-0.20}^{+0.10}$ & $17.09_{-0.23}^{+0.19}$ & $49.50_{-4.12}^{+2.55}$ & $20.34_{-0.69}^{+0.65}$ & $114.99_{-0.82}^{+1.52}$ & $0.59_{-0.23}^{+4.42}$ \\
\hline NGC 4319 & $3 \mathrm{~d}$ & 17.94 & 49.94 & $\ldots$ & $\ldots$ & $19.11_{-0.06}^{+0.20}$ & & $13.05_{-0.35}^{+1.11}$ & $\ldots$ & $\ldots$ & $\ldots$ \\
\hline NGC 4321 & 20 & 97.79 & 135.45 & 159.36 & 278.88 & $20.12_{-0.07}^{+0.09}$ & $18.77_{-0.21}^{+0.17}$ & $80.69_{-3.16}^{+4.51}$ & $48.57_{-2.50}^{+2.37}$ & $152.69_{-2.85}^{+3.56}$ & $1.51_{-0.55}^{+0.93}$ \\
\hline NGC 4351 & $3 d$ & 31.09 & 56.08 & 60.15 & 90.43 & $20.80_{-0.05}^{+0.03}$ & $21.45_{-0.19}^{+0.16}$ & $19.90_{-0.50}^{+0.44}$ & $25.09_{-2.00}^{+2.21}$ & $57.78_{-0.63}^{+1.05}$ & $3.55_{-0.90}^{+4.22}$ \\
\hline NGC 4355 & 1 & 41.00 & 62.92 & $\ldots$ & $\ldots$ & $19.84_{-0.23}^{+0.23}$ & $\ldots$ & $11.36_{-0.62}^{+0.71}$ & $\ldots$ & $\ldots$ & $\ldots$ \\
\hline NGC 4369 & $2 \mathrm{i}$ & 41.92 & 95.83 & $\ldots$ & $\ldots$ & $19.42_{-0.17}^{+0.12}$ & $\ldots$ & $14.78_{-0.61}^{+0.48}$ & $\ldots$ & $\ldots$ & $\ldots$ \\
\hline NGC 4380 & 20 & 40.80 & 58.89 & 68.57 & 158.17 & $20.72_{-0.03}^{+0.01}$ & $19.12_{-0.15}^{+0.12}$ & $57.93_{-1.87}^{+1.13}$ & $24.37_{-0.94}^{+0.84}$ & $62.05_{-1.42}^{+1.66}$ & $1.00_{-0.26}^{+2.34}$ \\
\hline NGC 4394 & 20 & 70.23 & 83.78 & 87.70 & 139.04 & $21.68_{-0.11}^{+0.07}$ & $18.81_{-0.33}^{+0.27}$ & $80.56_{-7.77}^{+4.55}$ & $22.90_{-1.61}^{+1.58}$ & $84.49_{-1.04}^{+1.10}$ & $2.62_{-1.25}^{+7.33}$ \\
\hline NGC 4405 & $3 d$ & 25.23 & 51.97 & 56.32 & 83.07 & $18.83_{-0.11}^{+0.13}$ & $21.50_{-0.51}^{+0.31}$ & $10.82_{-0.32}^{+0.32}$ & $21.53_{-3.35}^{+3.29}$ & $53.35_{-0.80}^{+0.67}$ & $1.15_{-0.43}^{+8.02}$ \\
\hline NGC 4413 & $2 i+3 d$ & 40.34 & 59.77 & 77.73 & 105.28 & $19.63_{-0.11}^{+0.21}$ & $21.19_{-0.17}^{+0.14}$ & $15.83_{-0.58}^{+1.02}$ & $25.41_{-1.51}^{+1.50}$ & $60.45_{-1.73}^{+1.84}$ & $0.28_{-0.04}^{+0.59}$ \\
\hline NGC 4424 & 1 & 78.58 & 147.24 & $\ldots$ & $\ldots$ & $20.67_{-0.18}^{+0.16}$ & & $32.54_{-2.03}^{+2.16}$ & & $\ldots$ & $\ldots$ \\
\hline NGC 4450 & 20 & 81.10 & 92.69 & 99.37 & 199.64 & $20.73_{-0.20}^{+0.15}$ & $19.26_{-0.12}^{+0.12}$ & $79.51_{-10.66}^{+10.80}$ & $38.03_{-1.35}^{+1.52}$ & $99.00_{-1.67}^{+2.21}$ & $1.04_{-0.37}^{+4.90}$ \\
\hline NGC 4457 & 20 & 95.77 & 121.28 & 129.46 & 151.12 & $21.71_{-0.19}^{+0.18}$ & $19.14_{-0.53}^{+0.51}$ & $52.61_{-4.05}^{+5.17}$ & $26.84_{-2.55}^{+3.47}$ & $129.74_{-1.68}^{+1.40}$ & $0.61_{-0.17}^{+3.88}$ \\
\hline NGC 4498 & $2 \mathrm{i}$ & 35.22 & 114.81 & $\ldots$ & $\ldots$ & $19.83_{-0.09}^{+0.08}$ & $\ldots$ & $19.18_{-0.57}^{+0.57}$ & $\ldots$ & $\ldots$ & $\ldots$ \\
\hline NGC 4501 & $3 d$ & 81.67 & 195.40 & 234.69 & 317.39 & $18.01_{-0.08}^{+0.05}$ & $21.08_{-0.39}^{+0.29}$ & $37.00_{-0.61}^{+0.43}$ & $70.60_{-7.13}^{+6.99}$ & $220.18_{-3.30}^{+2.69}$ & $0.13_{-0.03}^{+0.06}$ \\
\hline NGC 4548 & $20+3(d)$ & 94.83 & 116.04 & 123.53 & 170.94 & $20.21_{-0.11}^{+0.13}$ & $18.39_{-0.33}^{+0.32}$ & $54.90_{-0.69}^{+0.87}$ & $31.31_{-3.52}^{+4.21}$ & $122.65_{-1.89}^{+2.21}$ & $0.67_{-0.04}^{+0.18}$ \\
\hline NGC 4548 & $20+3(d)$ & 121.66 & 172.19 & 196.52 & 241.44 & $18.43_{-0.11}^{+0.13}$ & $20.34_{-0.33}^{+0.32}$ & $31.57_{-0.69}^{+0.87}$ & $45.79_{-3.52}^{+4.21}$ & $179.43_{-1.89}^{+2.21}$ & $0.21_{-0.04}^{+0.18}$ \\
\hline NGC 4579 & $2 \mathrm{i}$ & 95.53 & 259.29 & $\ldots$ & $\ldots$ & $18.96_{-0.12}^{+0.10}$ & $\ldots$ & $41.18_{-1.38}^{+1.28}$ & $\ldots$ & $\ldots$ & $\ldots$ \\
\hline NGC 4580 & 1 & 26.91 & 94.30 & $\ldots$ & $\ldots$ & $18.94_{-0.07}^{+0.06}$ & $\ldots$ & $15.16_{-0.32}^{+0.30}$ & $\ldots$ & $\ldots$ & $\ldots$ \\
\hline NGC 4639 & 20 & 54.87 & 73.78 & 78.97 & 98.62 & $20.16_{-0.08}^{+0.04}$ & $18.33_{-0.53}^{+0.36}$ & $22.23_{-0.64}^{+0.44}$ & $14.84_{-1.27}^{+1.12}$ & $75.37_{-0.74}^{+0.87}$ & $1.90_{-0.06}^{+4.73}$ \\
\hline NGC 4651 & $3 d$ & 52.15 & 117.58 & 125.45 & 169.73 & $19.66_{-0.13}^{+0.03}$ & $21.32_{-0.25}^{+0.23}$ & $26.87_{-0.76}^{+0.43}$ & $38.99_{-2.78}^{+3.83}$ & $131.92_{-3.22}^{+3.86}$ & $1.27_{-0.17}^{+2.96}$ \\
\hline NGC 4689 & $3 d$ & 28.50 & 104.36 & 109.62 & 154.35 & $20.17_{-0.04}^{+0.03}$ & $20.97_{-0.14}^{+0.19}$ & $34.33_{-0.90}^{+0.57}$ & $44.92_{-3.27}^{+4.51}$ & $107.31_{-1.38}^{+2.31}$ & $1.90_{-0.57}^{+7.94}$ \\
\hline NGC 4713 & 20 & 27.84 & 36.10 & 40.88 & 78.07 & $20.75_{-0.01}^{+0.06}$ & $18.63_{-0.10}^{+0.14}$ & $44.36_{-0.52}^{+3.71}$ & $13.36_{-0.34}^{+0.60}$ & $37.43_{-0.51}^{+0.40}$ & $10.51_{-5.20}^{+11.06}$ \\
\hline NGC 4725 & 20 & 238.86 & 278.79 & 303.74 & 384.31 & $21.73_{-0.17}^{+0.07}$ & $19.46_{-0.53}^{+0.47}$ & $130.27_{-10.48}^{+7.38}$ & $66.93_{-7.17}^{+8.82}$ & $288.01_{-2.18}^{+3.27}$ & $1.72_{-0.89}^{+1.25}$ \\
\hline NGC 4750 & 1 & 33.72 & 121.72 & $\ldots$ & $\ldots$ & $19.38_{-0.09}^{+0.07}$ & $\ldots$ & $20.75_{-0.64}^{+0.58}$ & $\ldots$ & $\ldots$ & $\ldots$ \\
\hline NGC 4772 & 20 & 84.53 & 110.68 & 122.36 & 148.98 & $21.64_{-0.13}^{+0.13}$ & $18.46_{-0.67}^{+0.48}$ & $61.74_{-4.65}^{+5.70}$ & $23.91_{-2.55}^{+2.62}$ & $114.24_{-1.59}^{+1.54}$ & $0.43_{-0.12}^{+1.72}$ \\
\hline NGC 4793 & $3 d$ & 26.29 & 47.33 & 54.90 & 88.97 & $17.62_{-0.14}^{+0.06}$ & $20.43_{-0.21}^{+0.30}$ & $10.37_{-0.31}^{+0.18}$ & $20.12_{-1.27}^{+1.97}$ & $55.28_{-1.14}^{+1.50}$ & $0.66_{-0.21}^{+3.08}$ \\
\hline NGC 4806 & 20 & 23.68 & 33.03 & 34.50 & 43.41 & $20.20_{-0.12}^{+0.06}$ & $15.82_{-0.42}^{+0.81}$ & $12.03_{-0.47}^{+0.32}$ & $4.92_{-0.24}^{+0.56}$ & $33.54_{-0.75}^{+0.39}$ & $3.40_{-1.35}^{+19.94}$ \\
\hline NGC 4826 & 20 & 72.01 & 171.83 & 236.00 & 347.23 & $18.25_{-0.03}^{+0.02}$ & $17.61_{-0.42}^{+0.25}$ & $56.70_{-0.84}^{+0.50}$ & $48.74_{-3.32}^{+2.57}$ & $203.13_{-18.14}^{+20.84}$ & $0.08_{-0.01}^{+0.52}$ \\
\hline NGC 4942 & 20 & 15.30 & 34.32 & 39.08 & 65.55 & $20.52_{-0.01}^{+0.01}$ & $19.61_{-0.23}^{+0.18}$ & $13.85_{-0.06}^{+0.06}$ & $10.28_{-0.53}^{+0.49}$ & $33.48_{-1.17}^{+0.87}$ & $6.37_{-1.06}^{+3.63}$ \\
\hline
\end{tabular}


Table 2

(Continued)

\begin{tabular}{|c|c|c|c|c|c|c|c|c|c|c|c|}
\hline Galaxy & Prof. Type & $\begin{array}{l}r_{1, i} \\
(") \\
(3)\end{array}$ & $\begin{array}{l}r_{2, i} \\
\left({ }^{\prime \prime}\right) \\
(4)\end{array}$ & $\begin{array}{l}r_{1, o} \\
\left({ }^{\prime \prime}\right) \\
(5)\end{array}$ & $\begin{array}{l}r_{2, o} \\
\left({ }^{\prime \prime}\right) \\
(6)\end{array}$ & $\begin{array}{c}\mu_{0, i} \\
(\mathrm{mag} / \square) \\
(7)\end{array}$ & $\begin{array}{c}\mu_{0, o} \\
(\mathrm{mag} / \square) \\
(8)\end{array}$ & $\begin{array}{l}h_{i} \\
\left({ }^{\prime \prime}\right) \\
(9)\end{array}$ & $\begin{array}{l}h_{o} \\
\left({ }^{\prime \prime}\right) \\
(10)\end{array}$ & $\begin{array}{l}R_{\mathrm{br}} \\
\left({ }^{\prime \prime}\right) \\
(11)\end{array}$ & $\begin{array}{c}\alpha \\
\left(I^{\prime \prime}\right) \\
(12)\end{array}$ \\
\hline NGC 4980 & 1 & 10.73 & 23.98 & $\cdots$ & $\cdots$ & $19.86_{-0.01}^{+0.07}$ & $\cdots$ & $10.16_{-0.09}^{+0.42}$ & $\cdots$ & $\cdots$ & . \\
\hline NGC 4984 & $20+2 o$ & 77.06 & 98.22 & 101.82 & 143.74 & $21.77_{-0.17}^{+0.18}$ & $20.31_{-1.20}^{+0.53}$ & $88.62_{-2.60}^{+3.40}$ & $39.57_{-4.94}^{+3.71}$ & $96.10_{-3.25}^{+4.59}$ & $5.12_{-0.35}^{+4.01}$ \\
\hline NGC 4984 & $20+2 o$ & 116.19 & 157.32 & 163.71 & 181.28 & $20.92_{-0.17}^{+0.18}$ & $18.90_{-1.20}^{+0.53}$ & $47.77_{-2.60}^{+3.40}$ & $30.70_{-4.94}^{+3.71}$ & $160.36_{-3.25}^{+4.59}$ & $0.78_{-0.35}^{+4.01}$ \\
\hline NGC 5055 & $3 \mathrm{~d}$ & 97.32 & 239.57 & 275.93 & 490.91 & $18.93_{-0.04}^{+0.06}$ & $20.45_{-0.14}^{+0.13}$ & $68.47_{-1.12}^{+1.56}$ & $111.73_{-5.59}^{+6.58}$ & $247.05_{-5.14}^{+5.21}$ & $0.14_{-0.05}^{+0.86}$ \\
\hline NGC 5068 & 20 & 86.09 & 147.59 & 155.08 & 235.29 & $20.72_{-0.07}^{+0.07}$ & $18.56_{-0.43}^{+0.37}$ & $65.27_{-2.35}^{+4.08}$ & $36.16_{-2.79}^{+3.40}$ & $161.24_{-3.79}^{+1.29}$ & $1.33_{-0.73}^{+3.84}$ \\
\hline NGC 5240 & $2 \mathrm{i}+2 \mathrm{o}$ & 23.68 & 54.67 & 59.29 & 72.38 & $19.84_{-0.02}^{+0.04}$ & $15.77_{-0.50}^{+0.59}$ & $13.36_{-0.08}^{+0.18}$ & $7.12_{-0.35}^{+0.52}$ & $57.17_{-0.85}^{+0.77}$ & $1.08_{-0.35}^{+8.62}$ \\
\hline NGC 5248 & 1 & 50.80 & 106.50 & $\ldots$ & $\ldots$ & $19.34_{-0.05}^{+0.05}$ & $\ldots$ & $35.46_{-0.83}^{+0.78}$ & $\ldots$ & . & $\ldots$ \\
\hline NGC 5273 & $2 \mathrm{i}$ & 39.23 & 99.73 & $\ldots$ & $\ldots$ & $19.27_{-0.13}^{+0.12}$ & $\ldots$ & $16.58_{-0.62}^{+0.66}$ & $\ldots$ & $\ldots$ & $\ldots$ \\
\hline NGC 5339 & $2 i+3 d$ & 40.38 & 57.21 & 61.42 & 82.66 & $18.23_{-0.13}^{+0.25}$ & $20.40_{-0.23}^{+0.45}$ & $11.06_{-0.30}^{+0.54}$ & $17.84_{-1.06}^{+2.34}$ & $58.13_{-0.81}^{+1.70}$ & $2.04_{-0.51}^{+5.59}$ \\
\hline NGC 5371 & 20 & 29.65 & 93.65 & 108.70 & 133.17 & $19.81_{-0.03}^{+0.01}$ & $13.69_{-1.28}^{+1.43}$ & $36.41_{-0.57}^{+0.42}$ & $12.92_{-1.36}^{+2.30}$ & $112.88_{-2.77}^{+1.90}$ & $0.33_{-0.07}^{+0.46}$ \\
\hline NGC 5377 & 20 & 86.66 & 112.74 & 119.05 & 168.69 & $21.95_{-0.10}^{+0.07}$ & $18.78_{-0.24}^{+0.24}$ & $70.65_{-3.34}^{+3.52}$ & $25.69_{-1.18}^{+1.32}$ & $117.71_{-0.86}^{+0.67}$ & $0.80_{-0.21}^{+3.24}$ \\
\hline NGC 5468 & $2 \mathrm{i}+2 \mathrm{o}+(3 \mathrm{~d})$ & 21.66 & 56.15 & 60.96 & 87.70 & $20.70_{-0.03}^{+0.09}$ & $18.63_{-0.16}^{+0.23}$ & $25.20_{-0.48}^{+0.67}$ & $13.73_{-0.45}^{+0.58}$ & $57.34_{-1.05}^{+0.34}$ & $2.94_{-0.21}^{+3.94}$ \\
\hline NGC 5480 & 1 & 31.53 & 78.94 & $\ldots$ & $\ldots$ & $19.55_{-0.11}^{+0.12}$ & $\ldots$ & $13.99_{-0.48}^{+0.55}$ & $\ldots$ & $\ldots$ & $\ldots$ \\
\hline NGC 5584 & 20 & 36.36 & 68.36 & 73.82 & 112.00 & $20.99_{-0.09}^{+0.07}$ & $19.47_{-0.27}^{+0.21}$ & $35.62_{-1.40}^{+1.36}$ & $21.21_{-1.38}^{+1.42}$ & $73.37_{-0.73}^{+1.37}$ & $2.57_{-0.23}^{+5.70}$ \\
\hline NGC 5585 & 20 & 81.93 & 132.20 & 139.74 & 178.95 & $22.17_{-0.10}^{+0.03}$ & $18.33_{-0.27}^{+0.34}$ & $69.03_{-2.65}^{+1.56}$ & $24.48_{-1.06}^{+1.50}$ & $133.96_{-1.07}^{+0.53}$ & $1.31_{-0.30}^{+3.18}$ \\
\hline NGC 5597 & $2 \mathrm{i}+3 \mathrm{~d}$ & 45.42 & 71.37 & 74.62 & 89.45 & $18.31_{-0.13}^{+0.15}$ & $20.74_{-0.17}^{+0.34}$ & $13.14_{-0.41}^{+0.39}$ & $22.17_{-1.33}^{+2.86}$ & $72.33_{-0.41}^{+0.73}$ & $3.78_{-1.11}^{+8.32}$ \\
\hline NGC 5645 & 20 & 26.07 & 52.14 & 57.20 & 79.98 & $20.28_{-0.06}^{+0.03}$ & $19.06_{-0.19}^{+0.22}$ & $19.04_{-0.49}^{+0.26}$ & $13.87_{-0.59}^{+0.73}$ & $57.41_{-0.71}^{+2.14}$ & $2.92_{-0.25}^{+5.48}$ \\
\hline NGC 5668 & 1 & 38.79 & 103.76 & $\ldots$ & $\ldots$ & $20.60_{-0.11}^{+0.10}$ & $\ldots$ & $23.02_{-1.13}^{+1.11}$ & $\ldots$ & $\ldots$ & $\ldots$ \\
\hline NGC 5669 & 20 & 43.08 & 64.62 & 69.91 & 114.50 & $21.62_{-0.25}^{+0.11}$ & $20.44_{-0.15}^{+0.15}$ & $41.92_{-5.53}^{+3.04}$ & $25.16_{-1.22}^{+1.58}$ & $68.22_{-0.46}^{+5.54}$ & $3.17_{-0.71}^{+5.63}$ \\
\hline NGC 5713 & $3 d$ & 28.45 & 55.54 & 60.69 & 93.20 & $18.50_{-0.12}^{+0.07}$ & $19.69_{-0.11}^{+0.09}$ & $14.19_{-0.49}^{+0.27}$ & $19.93_{-0.67}^{+0.59}$ & $53.92_{-1.39}^{+0.92}$ & $1.52_{-0.66}^{+5.25}$ \\
\hline NGC 5740 & $2 \mathrm{i}$ & 30.97 & 90.24 & $\ldots$ & $\ldots$ & $18.68_{-0.12}^{+0.10}$ & $\ldots$ & $14.38_{-0.48}^{+0.45}$ & $\ldots$ & $\ldots$ & $\ldots$ \\
\hline NGC 5762 & $3 \mathrm{~d}$ & 20.83 & 36.57 & 39.03 & 58.71 & $21.44_{-0.13}^{+0.06}$ & $22.62_{-0.18}^{+0.10}$ & $12.60_{-0.61}^{+0.35}$ & $20.35_{-1.78}^{+1.43}$ & $36.10_{-1.54}^{+0.39}$ & $7.23_{-3.07}^{+13.48}$ \\
\hline NGC 5806 & 1 & 53.40 & 140.38 & $\ldots$ & $\ldots$ & $20.02_{-0.14}^{+0.11}$ & $\ldots$ & $28.00_{-1.35}^{+1.28}$ & $\ldots$ & $\ldots$ & $\ldots$ \\
\hline NGC 5850 & 20 & 97.45 & 127.03 & 138.67 & 188.60 & $21.82_{-0.23}^{+0.06}$ & $19.57_{-0.42}^{+0.29}$ & $73.54_{-8.48}^{+3.53}$ & $34.29_{-3.03}^{+2.74}$ & $132.94_{-1.40}^{+3.09}$ & $0.43_{-0.15}^{+2.78}$ \\
\hline NGC 5892 & 20 & 29.52 & 83.21 & 94.12 & 106.31 & $21.02_{-0.04}^{+0.10}$ & $16.80_{-1.02}^{+2.39}$ & $28.09_{-0.59}^{+1.53}$ & $13.08_{-1.41}^{+6.10}$ & $95.17_{-14.09}^{+1.44}$ & $0.46_{-0.12}^{+0.99}$ \\
\hline NGC 5915 & 20 & 21.03 & 40.17 & 43.96 & 55.00 & $19.29_{-0.20}^{+0.08}$ & $15.59_{-1.58}^{+1.00}$ & $11.42_{-0.39}^{+0.33}$ & $5.92_{-0.86}^{+0.62}$ & $41.96_{-1.00}^{+0.89}$ & $4.81_{-0.85}^{+10.51}$ \\
\hline NGC 5949 & 20 & 14.15 & 23.96 & 27.84 & 84.65 & $19.63_{-0.04}^{+0.04}$ & $18.18_{-0.12}^{+0.11}$ & $25.58_{-1.06}^{+1.52}$ & $11.53_{-0.33}^{+0.33}$ & $27.97_{-0.92}^{+1.09}$ & $1.29_{-0.46}^{+8.71}$ \\
\hline NGC 5950 & 20 & 20.90 & 36.10 & 41.95 & 55.84 & $21.08_{-0.05}^{+0.02}$ & $17.59_{-0.51}^{+0.22}$ & $19.72_{-0.57}^{+0.30}$ & $7.81_{-0.55}^{+0.32}$ & $41.57_{-0.32}^{+0.76}$ & $0.85_{-0.22}^{+0.88}$ \\
\hline NGC 5958 & $2 \mathrm{i}+3(\mathrm{~d})$ & 15.15 & 35.32 & 37.96 & 54.78 & $18.95_{-0.13}^{+0.07}$ & $21.83_{-0.63}^{+0.18}$ & $7.61_{-0.23}^{+0.21}$ & $17.22_{-3.56}^{+2.44}$ & $36.13_{-0.67}^{+0.51}$ & $1.89_{-0.76}^{+17.49}$ \\
\hline NGC 5964 & 20 & 51.76 & 87.14 & 94.04 & 136.31 & $21.70_{-0.07}^{+0.11}$ & $20.18_{-0.26}^{+0.22}$ & $52.06_{-2.44}^{+3.40}$ & $28.74_{-2.15}^{+2.13}$ & $89.96_{-1.24}^{+0.99}$ & $2.65_{-0.20}^{+4.71}$ \\
\hline NGC 5985 & 20 & 46.36 & 53.64 & 57.27 & 149.70 & $20.23_{-0.83}^{+0.29}$ & $18.75_{-0.16}^{+0.11}$ & $63.38_{-28.58}^{+38.36}$ & $25.86_{-0.98}^{+0.94}$ & $59.49_{-2.70}^{+13.44}$ & $1.38_{-0.69}^{+8.71}$ \\
\hline NGC 6012 & $3 \mathrm{~d}+(2 \mathrm{o})$ & 29.03 & 59.74 & 63.10 & 95.70 & $19.62_{-0.14}^{+0.06}$ & $22.32_{-0.27}^{+0.15}$ & $15.11_{-0.57}^{+0.34}$ & $39.67_{-4.63}^{+4.50}$ & $60.68_{-1.14}^{+0.50}$ & $3.18_{-0.25}^{+5.58}$ \\
\hline NGC 6014 & 1 & 24.16 & 60.69 & $\ldots$ & $\ldots$ & $20.09_{-0.06}^{+0.06}$ & $\ldots$ & $13.85_{-0.29}^{+0.34}$ & $\ldots$ & $\ldots$ & $\ldots$ \\
\hline NGC 6063 & $20+2 o$ & 16.59 & 25.58 & 27.20 & 42.17 & $20.07_{-0.09}^{+0.10}$ & $18.97_{-0.25}^{+0.28}$ & $15.63_{-0.24}^{+0.27}$ & $10.12_{-0.28}^{+0.36}$ & $28.93_{-0.50}^{+0.24}$ & $3.09_{-1.63}^{+28.97}$ \\
\hline NGC 6063 & $20+2 o$ & 26.08 & 41.18 & 42.55 & 53.28 & $19.20_{-0.09}^{+0.10}$ & $17.22_{-0.25}^{+0.28}$ & $10.64_{-0.24}^{+0.27}$ & $7.34_{-0.28}^{+0.36}$ & $43.03_{-0.50}^{+0.24}$ & $7.30_{-1.63}^{+28.97}$ \\
\hline NGC 6106 & 1 & 28.27 & 80.71 & $\cdots$ & $\ldots$ & $19.35_{-0.09}^{+0.09}$ & $\ldots$ & $14.27_{-0.45}^{+0.51}$ & $\ldots$ & $\ldots$ & $\ldots$ \\
\hline NGC 6140 & 20 & 41.57 & 86.10 & 91.02 & 105.77 & $21.67_{-0.06}^{+0.13}$ & $18.85_{-0.82}^{+0.50}$ & $37.22_{-1.47}^{+2.63}$ & $18.28_{-2.36}^{+2.10}$ & $93.43_{-1.44}^{+1.41}$ & $2.03_{-0.20}^{+4.38}$ \\
\hline NGC 6155 & 1 & 21.32 & 55.76 & $\ldots$ & $\ldots$ & $19.03_{-0.12}^{+0.09}$ & $\ldots$ & $8.90_{-0.30}^{+0.26}$ & $\ldots$ & .. & $\ldots$ \\
\hline NGC 6181 & 20 & 37.19 & 50.88 & 54.99 & 75.29 & $19.30_{-0.25}^{+0.08}$ & $16.19_{-0.26}^{+0.28}$ & $17.86_{-1.38}^{+0.51}$ & $8.98_{-0.31}^{+0.40}$ & $51.80_{-0.67}^{+0.74}$ & $1.22_{-0.39}^{+6.21}$ \\
\hline NGC 6207 & $3 \mathrm{~s}$ & 22.14 & 75.85 & $\ldots$ & $\ldots$ & $18.68_{-0.05}^{+0.04}$ & $\ldots$ & $13.73_{-0.16}^{+0.16}$ & $\ldots$ & $\ldots$ & $\ldots$ \\
\hline NGC 6217 & 20 & 63.40 & 85.73 & 89.33 & 104.21 & $20.48_{-0.20}^{+0.11}$ & $16.91_{-\ldots}^{+1.38}$ & $21.38_{-1.33}^{+0.96}$ & $12.09_{-5.97}^{+2.63}$ & $91.61_{-0.89}^{+1.11}$ & $3.53_{-0.36}^{+5.59}$ \\
\hline NGC 6267 & $2 \mathrm{i}+3 \mathrm{~d}$ & 27.55 & 42.07 & 44.92 & 63.34 & $17.86_{-0.24}^{+0.31}$ & $20.35_{-0.36}^{+0.48}$ & $8.37_{-0.37}^{+0.48}$ & $13.99_{-1.27}^{+2.00}$ & $47.94_{-1.28}^{+1.89}$ & $1.75_{-0.59}^{+9.37}$ \\
\hline NGC 6278 & 20 & 22.16 & 35.49 & 47.45 & 65.69 & $19.68_{-0.14}^{+0.06}$ & $17.85_{-0.37}^{+0.26}$ & $15.76_{-1.12}^{+0.61}$ & $9.09_{-0.59}^{+0.49}$ & $36.17_{-1.42}^{+2.18}$ & $0.42_{-0.06}^{+0.16}$ \\
\hline NGC 6339 & $2 \mathrm{i}+2 \mathrm{o}$ & 38.02 & 69.18 & 72.50 & 99.24 & $20.95_{-0.07}^{+0.11}$ & $18.78_{-0.37}^{+0.31}$ & $26.46_{-0.85}^{+1.04}$ & $15.07_{-1.02}^{+0.93}$ & $69.96_{-1.10}^{+0.78}$ & $3.23_{-0.96}^{+7.41}$ \\
\hline NGC 6412 & 20 & 20.91 & 34.93 & 38.62 & 74.29 & $20.98_{-0.08}^{+0.04}$ & $18.93_{-0.08}^{+0.08}$ & $45.28_{-4.67}^{+2.65}$ & $14.06_{-0.34}^{+0.35}$ & $38.57_{-0.24}^{+0.75}$ & $5.15_{-0.45}^{+6.79}$ \\
\hline NGC 6434 & 1 & 24.11 & 58.30 & $\ldots$ & $\ldots$ & $19.05_{-0.12}^{+0.10}$ & $\ldots$ & $10.76_{-0.40}^{+0.37}$ & $\ldots$ & $\ldots$ & $\ldots$ \\
\hline NGC 6923 & $2 \mathrm{i}$ & 28.84 & 90.51 & $\cdots$ & $\ldots$ & $18.41_{-0.09}^{+0.10}$ & $\ldots$ & $13.16_{-0.33}^{+0.37}$ & $\cdots$ & $\cdots$ & $\ldots$ \\
\hline NGC 7098 & 20 & 111.98 & 159.15 & $\ldots$ & $\ldots$ & $18.89_{-0.50}^{+0.36}$ & $\ldots$ & $27.68_{-2.55}^{+2.32}$ & $\ldots$ & $\ldots$ & $\ldots$ \\
\hline NGC 7140 & 20 & 88.80 & 121.68 & 129.62 & 142.47 & $22.23_{-0.23}^{+0.11}$ & $15.83_{-\ldots}^{+1.92}$ & $50.73_{-4.63}^{+3.68}$ & $15.25_{-6.57}^{+4.40}$ & $128.47_{-1.72}^{+6.65}$ & $0.63_{-0.15}^{+3.44}$ \\
\hline NGC 7479 & 20 & 64.84 & 99.80 & 107.61 & 136.13 & $19.90_{-0.09}^{+0.04}$ & $15.77_{-0.75}^{+0.56}$ & $37.54_{-1.47}^{+0.79}$ & $15.51_{-1.36}^{+1.31}$ & $100.44_{-1.42}^{+1.31}$ & $0.64_{-0.09}^{+4.41}$ \\
\hline NGC 7552 & 20 & 64.89 & 88.60 & 95.20 & 116.58 & $20.69_{-0.06}^{+0.07}$ & $15.82_{-0.69}^{+0.46}$ & $37.72_{-1.09}^{+1.35}$ & $13.38_{-1.07}^{+0.91}$ & $93.07_{-0.60}^{+0.67}$ & $0.76_{-0.22}^{+6.59}$ \\
\hline NGC 7625 & 1 & 27.40 & 58.38 & $\ldots$ & $\ldots$ & $18.77_{-0.12}^{+0.11}$ & $\ldots$ & $9.66_{-0.30}^{+0.33}$ & $\ldots$ & $\ldots$ & $\ldots$ \\
\hline
\end{tabular}


Table 2

(Continued)

\begin{tabular}{|c|c|c|c|c|c|c|c|c|c|c|c|}
\hline Galaxy & (2) & $\begin{array}{l}r_{1, i} \\
\left({ }^{\prime \prime}\right) \\
(3)\end{array}$ & $\begin{array}{l}r_{2, i} \\
\left({ }^{\prime \prime}\right) \\
(4)\end{array}$ & $\begin{array}{l}r_{1, o} \\
\left({ }^{\prime \prime}\right) \\
(5)\end{array}$ & $\begin{array}{l}r_{2, o} \\
(") \\
(6)\end{array}$ & $\begin{array}{c}\mu_{0, i} \\
(\mathrm{mag} / \square) \\
\text { (7) }\end{array}$ & $\begin{array}{c}\mu_{0, o} \\
(\mathrm{mag} / \square) \\
(8)\end{array}$ & $\begin{array}{l}h_{i} \\
\left({ }^{\prime \prime}\right) \\
(9)\end{array}$ & $\begin{array}{c}h_{o} \\
\left({ }^{\prime \prime}\right) \\
(10)\end{array}$ & $\begin{array}{c}R_{\mathrm{br}} \\
\left({ }^{\prime \prime}\right) \\
(11)\end{array}$ & $\begin{array}{c}\alpha \\
\left(/^{\prime \prime}\right) \\
(12)\end{array}$ \\
\hline NGC 7661 & 20 & 25.88 & 45.69 & 50.00 & 62.16 & $22.23_{-0.05}^{+0.05}$ & $17.82_{-0.37}^{+0.33}$ & $28.92_{-1.21}^{+1.37}$ & $8.19_{-0.45}^{+0.47}$ & $46.35_{-0.27}^{+0.32}$ & $1.16_{-0.35}^{+5.62}$ \\
\hline NGC 7741 & $2 i+2 o$ & 50.80 & 107.70 & 113.80 & 132.08 & $20.45_{-0.05}^{+0.06}$ & $16.29_{-0.68}^{+0.67}$ & $32.06_{-0.88}^{+0.81}$ & $14.91_{-1.09}^{+1.36}$ & $106.79_{-2.08}^{+1.80}$ & $0.82_{-0.33}^{+5.37}$ \\
\hline NGC 7742 & $3(\mathrm{~s})$ & 30.23 & 50.89 & 62.85 & 85.46 & $19.36_{-0.07}^{+0.08}$ & $21.30_{-0.24}^{+0.18}$ & $12.42_{-0.27}^{+0.30}$ & $21.11_{-1.68}^{+1.69}$ & $53.80_{-0.84}^{+0.61}$ & $0.42_{-0.08}^{+0.18}$ \\
\hline PGC006667 & $1+(20)$ & 28.86 & 78.06 & $\cdots$ & $\ldots$ & $21.36_{-0.08}^{+0.07}$ & $\ldots$ & $21.71_{-0.76}^{+0.82}$ & $\ldots$ & $\ldots$ & $\ldots$ \\
\hline PGC011367 & $2 \mathrm{i}$ & 33.53 & 70.72 & $\ldots$ & $\ldots$ & $19.95_{-0.16}^{+0.17}$ & $\cdots$ & $12.75_{-0.61}^{+0.70}$ & $\cdots$ & $\ldots$ & $\cdots$ \\
\hline PGC012633 & 1 & 27.81 & 45.41 & $\ldots$ & $\ldots$ & $19.87_{-0.27}^{+0.21}$ & $\ldots$ & $9.35_{-0.69}^{+0.66}$ & $\cdots$ & $\ldots$ & $\cdots$ \\
\hline PGC012664 & 1 & 27.81 & 63.46 & $\ldots$ & $\ldots$ & $21.66_{-0.14}^{+0.08}$ & $\ldots$ & $18.72_{-1.32}^{+1.06}$ & $\ldots$ & $\ldots$ & $\ldots$ \\
\hline PGC014037 & $2 \mathrm{i}+2 \mathrm{o}$ & 13.34 & 23.61 & 27.90 & 34.34 & $20.30_{-0.07}^{+0.07}$ & $18.12_{-0.48}^{+0.59}$ & $8.26_{-0.21}^{+0.26}$ & $5.16_{-0.37}^{+0.57}$ & $27.49_{-1.35}^{+0.56}$ & $1.17_{-0.27}^{+0.73}$ \\
\hline UGC03070 & 1 & 13.12 & 40.01 & $\ldots$ & $\ldots$ & $20.86_{-0.10}^{+0.07}$ & $\ldots$ & $10.53_{-0.62}^{+0.55}$ & $\ldots$ & $\ldots$ & $\ldots$ \\
\hline UGC09356 & $2 i+3 d$ & 10.11 & 20.59 & 22.46 & 35.94 & $19.50_{-0.01}^{+0.04}$ & $20.29_{-0.07}^{+0.10}$ & $7.65_{-0.06}^{+0.12}$ & $10.13_{-0.34}^{+0.44}$ & $22.51_{-0.09}^{+0.39}$ & $8.06_{-3.43}^{+14.88}$ \\
\hline UGC10437 & $3 d$ & 9.30 & 19.57 & 21.34 & 55.35 & $20.61_{-0.03}^{+0.01}$ & $22.43_{-0.07}^{+0.05}$ & $7.09_{-0.09}^{+0.06}$ & $17.48_{-0.93}^{+0.96}$ & $19.87_{-0.18}^{+0.11}$ & $9.45_{-0.97}^{+13.22}$ \\
\hline UGC10445 & $2 o+3 d$ & 20.11 & 34.04 & 36.47 & 53.93 & $22.12_{-0.07}^{+0.12}$ & $20.26_{-0.22}^{+0.13}$ & $32.90_{-0.30}^{+0.41}$ & $12.69_{-1.90}^{+1.59}$ & $35.38_{-0.82}^{+0.21}$ & $6.65_{-0.73}^{+3.73}$ \\
\hline UGC10445 & $2 o+3 d$ & 35.14 & 51.94 & 56.36 & 74.49 & $20.25_{-0.07}^{+0.12}$ & $22.23_{-0.22}^{+0.13}$ & $12.66_{-0.30}^{+0.41}$ & $22.30_{-1.90}^{+1.59}$ & $53.53_{-0.82}^{+0.21}$ & $5.71_{-0.73}^{+3.73}$ \\
\hline
\end{tabular}

Notes. Structural properties of the disk profiles. (1) Galaxy name. (2) Profile type (see Section 3.2). Terms between brackets denote an uncertain classification. (3) and (4) Radial limits of the inner exponential. (5) and (6) Radial limits of the outer exponential. (7) and (8) Extrapolated central surface brightness of the inner and outer exponentials. No inclination correction has been applied. (9) and (10) Radial scale length of the inner and outer exponentials. (11) Break radius. (12) Break sharpness: large values correspond to abrupt breaks and low values to smoother ones.

(This table is also available in a machine-readable form in the online journal.)

fit. In practice, for each galaxy we compute a critical surface brightness value $\mu_{\text {crit }}$ beyond which the uncertainty in the light profile exceeds $0.2 \mathrm{mag} \operatorname{arcsec}^{-2}$. We typically place the outer boundary around the radius where this surface brightness is reached.

Type II and III profiles require a more sophisticated approach. In order to simultaneously fit the inner and outer disks, we rely on the broken exponential function proposed by E08:

$$
I(r)=S I_{0} e^{-\frac{r}{h_{i}}}\left[1+e^{\alpha\left(r-R_{\mathrm{br}}\right)}\right]^{\frac{1}{\alpha}\left(\frac{1}{h_{i}}-\frac{1}{h_{o}}\right)} .
$$

Here, $h_{i}$ and $h_{o}$ are the scale lengths of the inner and outer disks, respectively, $I_{0}$ is the central intensity of the inner disk, and $R_{\mathrm{br}}$ is the break radius. The coefficient $\alpha$ determines the sharpness of the break, with low values corresponding to a smooth transition between the inner and outer disks, and high values yielding a sharper break. The scaling factor $S$ is defined as ${ }^{23}$

$$
S=\left(1+e^{-\alpha R_{\mathrm{br}}}\right)^{\frac{1}{\alpha}\left(\frac{1}{h_{o}}-\frac{1}{h_{i}}\right)} .
$$

An example of such a fit is shown in Figure 3 for NGC 0936. We first identify the inner and outer portions of the disk profile, and delimit them with two points each (shown here as small squares). Again, the innermost limit of all is set to avoid contamination from the bulge and bar, whereas the outermost one is placed in general around $\mu_{\text {crit }}$. We then apply individual linear fits to each portion of the profile separately. From these

\footnotetext{
23 Note that the definition of $S$ in E08 (their Equation (6)) contains a small
} typo: the order of the $1 / h_{i}$ and $1 / h_{o}$ terms should be reversed. we get initial estimates for both slopes and $y$-intercepts, as well as for the break radius (from the intersection of both fits). The resulting values are then used as input guesses for the parameters in Equation (5), which is fitted iteratively to all data points bracketed by the innermost and outermost boundaries, using a Levenberg-Marquardt algorithm.

The final values of the fitted parameters might depend on our particular placement of the four range delimiters, as well as on the accuracy of the sky subtraction. In order to get a handle on the uncertainties that these factors might introduce, we proceed in a Monte Carlo fashion. For a given profile we generate 1000 simulations, in each of which both the range delimiters and the sky value are randomly modified at the same time. The delimiters are shuffled around their central positions following a uniform probability distribution, with a half-width equal to $\pm 0.05 \times R_{25.5}$. This interval is shown in Figure 3 as horizontal error bars. In some cases this might place the innermost delimiter somewhat inside the bulge- or bar-dominated region of the profile, but we do actually want to include this in the error budget. Also, note that by using a fractional value of the total disk size, we allow for a proportionally larger margin of error in galaxies with larger apparent sizes. As for the sky level, we draw random values from a Gaussian distribution whose standard deviation matches the large-scale background rms measured in the image (as explained in Section 3.1), which is the main source of uncertainty in $\mu$ at large radii. After automatically fitting each simulated profile, we end up with a set of 1000 values for each one of the structural parameters. Out of these distributions we get the corresponding upper and lower $1 \sigma$ uncertainties, which are quoted in Table 2. 


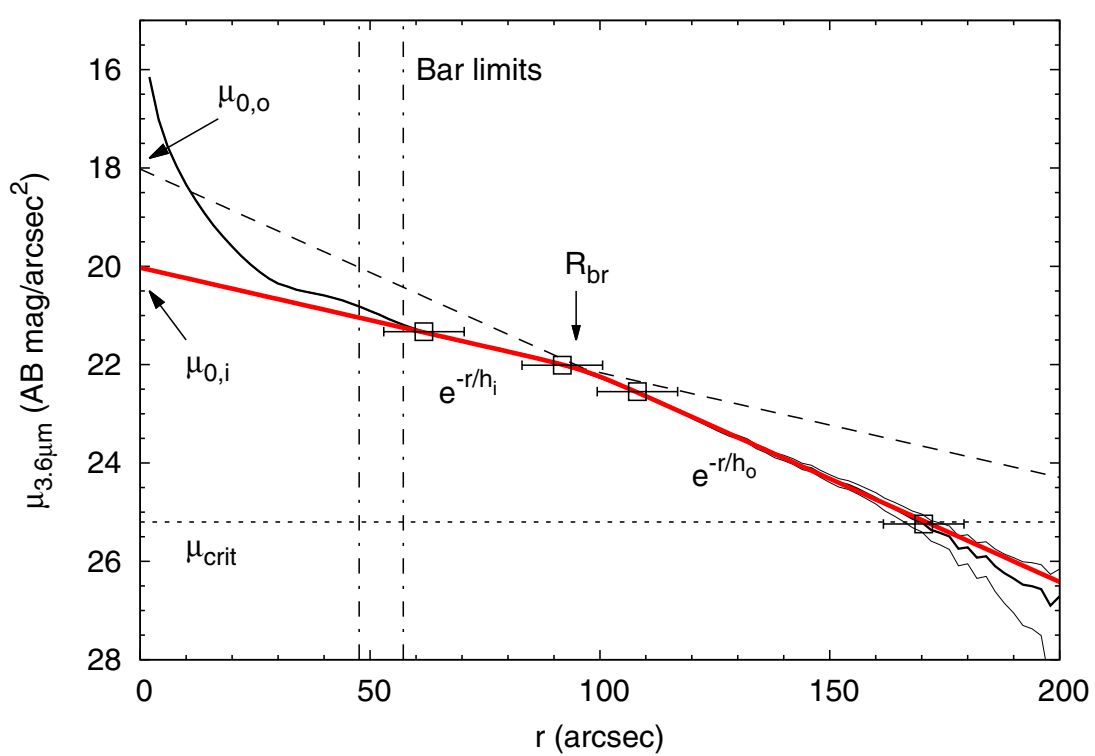

Figure 3. Sample fit of the broken exponential function to the light profile of NGC 0936, showing the nomenclature used throughout the paper. The profile itself is shown as a thick black line, whereas the thinner lines above and below it show the $\pm 1 \sigma$ uncertainty. The four squares delimit the inner and outer portions of the disk, from which initial guesses of the slopes and central surface brightnesses are obtained. Their error bars delimit the range used in the Monte Carlo simulations. The best-fitting broken exponential described by Equation (5) appears in red. Even though this red curve is plotted spanning the whole radial range, the fit itself is done only between the first and fourth square points. Dashed lines show extrapolations of the inner and outer disks. The dotted line marks the critical surface brightness level $\mu_{\text {crit }}$, beyond which $\Delta \mu>0.2 \mathrm{mag} \operatorname{arcsec}^{-2}$. Finally, vertical dot-dashed lines mark the minimum and maximum of our estimates of the bar radius (Section 3.4). (A color version of this figure is available in the online journal.)

\subsection{Measuring Bar Properties}

The potential impact of a bar in shaping a galactic disk might depend, at least to first order, on its length and its strength. Longer bars have access to more material within the disk, and can therefore be responsible for the galaxy-wide radial rearrangement of both stars and gas (which in turn might eventually be converted into new stars). Bar strength, on the other hand, measures the non-axisymmetric torque exerted by the bar. It is usually defined as the ratio between the maximum tangential force normalized by the mean axisymmetric radial force inside (Combes \& Sanders 1981). Obtaining the bar strength directly from this definition requires a detailed evaluation of the gravitational potential of the galaxy. This can be done from a galaxy's IR image, provided that some assumptions on the mass-to-light ratio and disk scale height are made (see, e.g., Quillen et al. 1994; Buta \& Block 2001; Laurikainen \& Salo 2002). Such an analysis is beyond the scope of this paper; however, it has been shown that the maximum bar ellipticity constitutes a reasonable proxy for the bar strength (Laurikainen et al. 2002; Comerón et al. 2010). In general, bars with larger ellipticities tend to be stronger and exert greater torques.

Several methods and criteria have been proposed by different authors to identify bars and measure their structural properties (length, ellipticity, and P.A.). Among the most widely used techniques are those based on ellipse fitting (Wozniak et al. 1995; Knapen et al. 2000; Athanassoula \& Misiriotis 2002; Laine et al. 2002; Sheth et al. 2002; Erwin 2005; Gadotti et al. 2007; Menéndez-Delmestre et al. 2007). Other methodologies have also been used extensively, such as direct visual measurements (Kormendy 1979; Martin 1995; Hoyle et al. 2011), 2D image decompositions (Prieto et al. 2001; Peng et al. 2002; de Souza et al. 2004; Laurikainen et al. 2005; Reese et al. 2007; Gadotti 2008; T. Kim et al., in preparation), Fourier analysis (Ohta et al. 1990; Elmegreen \& Elmegreen 1985; Buta et al. 2006; Athanassoula \& Misiriotis 2002; Laurikainen et al. 2007), and cuts along the bar major axis (Athanassoula \& Misiriotis 2002; Elmegreen \& Elmegreen 1985).

Here, we rely on our radial profiles to determine the structural properties of bars. Figure 4 depicts the typical signature left by a bar on the ellipticity and P.A. profiles. Within the bar region, the ellipticity increases monotonically and then decreases by $\Delta \epsilon>0.1$ as the isophotes begin to probe the disk. The P.A., on the other hand, remains roughly constant along the bar (except perhaps at the center), and then changes by $\Delta$ P.A. $\gtrsim 10^{\circ}$ at the bar ends. How abrupt these changes in $\epsilon$ and P.A. are depend on how the spiral arms merge with the bar ends, as well as on the orientation of the bar relative to the projected disk (see Menéndez-Delmestre et al. 2007 for examples and a detailed discussion).

Bars in our sample were identified by simultaneously inspecting the images and profiles for each galaxy, looking for the signatures described above. Those cases where the presence of a bar was dubious were tagged as "candidate bars." These usually correspond to cases where the profiles exhibit a signature reminiscent of a bar, but where the image does not convincingly confirm its presence. In particular, some late-type disks exhibit elongated structures that may result from a chance alignment of a few bright $\mathrm{H}$ II regions. Also, non-axisymmetric bulges may be present in many galaxies (e.g., Zaritsky \& Lo 1986)

After several trials and experiments, and building on previous work, we decided to settle on four different measurements of the bar radius based on the ellipticity and P.A. profiles.

1. $a_{\epsilon \max }$, the radius where the ellipticity of the bar is maximum.

2. $a_{\epsilon}$ min, the radius where there is a local minimum in ellipticity after the previous maximum.

3. $a_{\Delta \epsilon=0.1}$, the radius where the ellipticity drops by 0.1 with respect to the maximum one.

4. $a_{\Delta \mathrm{P} . \mathrm{A} .}=10^{\circ}$, the radius where the P.A. differs by $10^{\circ}$ from the one at $a_{\epsilon \max }$. 

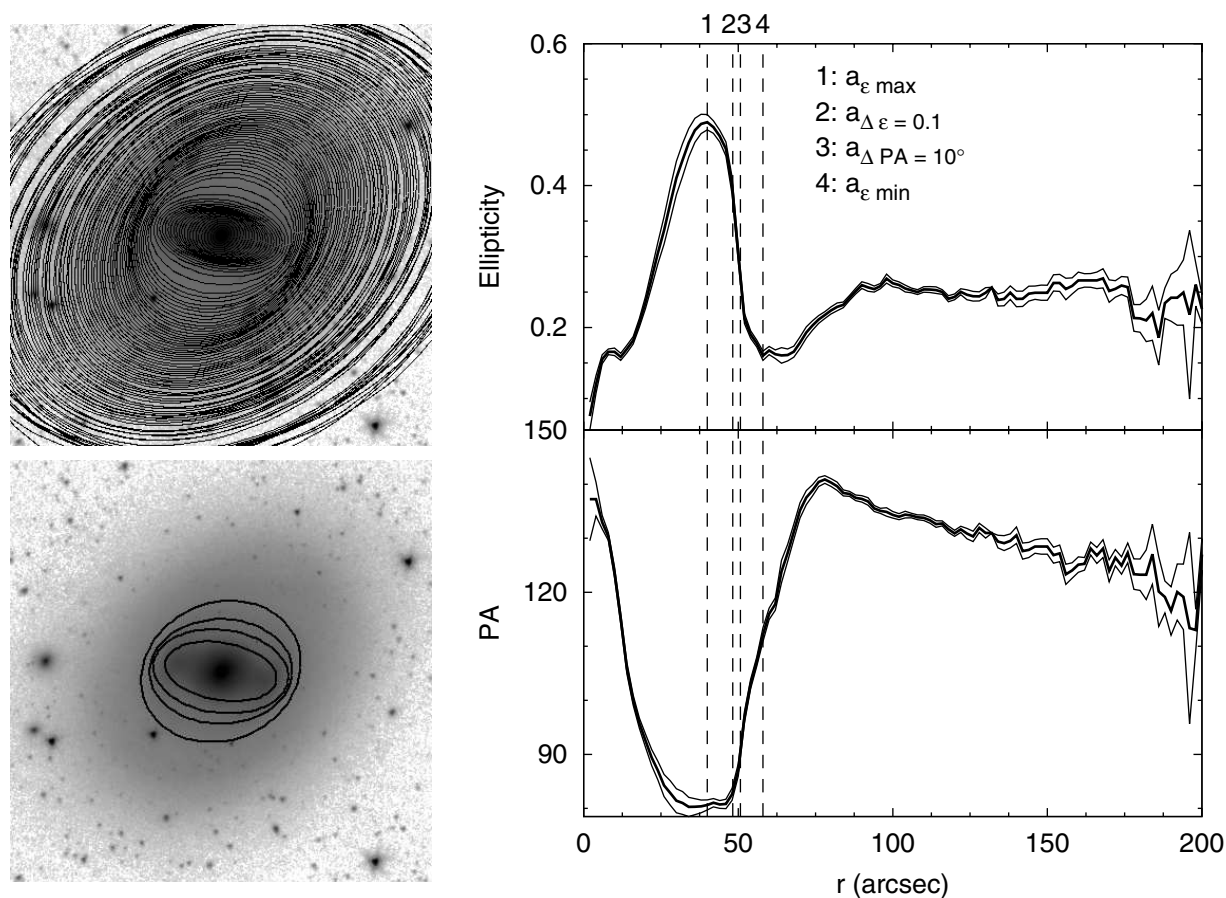

Figure 4. Signature left by a bar in the ellipticity and position angle profiles. The top-left panel shows the $3.6 \mu \mathrm{m}$ image of NGC 0936 with the ellipse fitting results overlaid with a spacing of $2^{\prime \prime}$ along the major axis between adjacent isophotes. The ellipticity and position angle profiles are displayed to the right; the thin lines above and below each profile show the $\pm 1 \sigma$ fitting error. The four estimations of the bar radius described in the text are marked with vertical dashed lines, and the corresponding four ellipses are overlaid in the bottom-left panel.

These four measurements are shown in Figure 4. Some observations (e.g., Wozniak et al. 1995) and $N$-body simulations (e.g., Athanassoula \& Misiriotis 2002) suggest that $a_{\epsilon \max }$ underestimates the true radius of the bar. We therefore adopt $a_{\epsilon \max }$ as a lower limit for the bar radius. As for the upper limit, we take whichever of the three remaining measurements is smallest. ${ }^{24}$ On average, after deprojection (see below) the upper limits are $20 \%$ larger than the lower ones, in agreement with Erwin et al. (2005). Our final bar radius for each galaxy is obtained by averaging the corresponding lower and upper limits. The bar ellipticity and P.A. are assumed to be those at $a_{\epsilon \max }$. These different measurements, together with the final adopted values, are quoted in Table 3.

All these three bar properties-radius, $\epsilon$, and P.A.-need to be corrected for inclination to obtain the intrinsic faceon values. To do so, we follow the 2D deprojection method described in Gadotti et al. (2007), which yields the true major and minor axes and P.A. of an ellipse seen in projection. This constitutes an improvement over simpler methods whereby bars are assumed to be one-dimensional lines. According to Gadotti et al. (2007), the geometrical parameters resulting from this analytical deprojection agree well with those measured on deprojected images.

This method requires knowing the line of nodes of the projected disk and its inclination angle. We derive these values from the P.A. and $\epsilon$ at $25.5 \mathrm{AB}$ mag $\operatorname{arcsec}^{-2}$, assuming that the outer disk at this surface brightness level is intrinsically circular. Note that kinematic P.A. and inclinations do not exist for such a large sample of galaxies, so this photometric approach is a

\footnotetext{
${ }^{24}$ Note that, in principle, it would not be necessary to compute $a_{\epsilon} \min$, since $a_{\Delta \epsilon=0.1}$ will generally be smaller, almost by definition. However, we did find a few galaxies for which the ellipticity drop at the end of the bar was smaller than (but very close to) 0.1 . In these few cases, $a_{\epsilon} \min$ was found to be a much better measure of the bar radius.
}

simple yet necessary work around in order to treat the whole sample homogeneously.

The intrinsic thickness of the disk is not expected to represent an important issue here, either, because most of our galaxies are just moderately inclined $(b / a>0.5)$. If a galaxy is described as an oblate spheroid (Hubble 1926), then the "true" inclination angle $i$ can be estimated as

$$
\cos ^{2} i=\left(q^{2}-q_{0}^{2}\right)\left(1-q_{0}^{2}\right)^{-1},
$$

where $q=b / a$ is the observed axial ratio and $q_{0}$ is the intrinsic one when the galaxy is viewed edge-on. Values of $q_{0} \simeq 0.2$ are usually assumed in the near-IR (e.g., Courteau et al. 2007 in the Two Micron All Sky Survey bands) and the mid-IR (e.g., Comerón et al. 2011a at $3.6 \mu \mathrm{m}$ ). For samples with $b / a>0.5$ such as ours, disk thickness should not bias the assumed inclination angles by more than $\sim 2^{\circ}$.

\section{RESULTS}

\subsection{Global Properties of Down-bending Profiles}

Before diving into an in-depth analysis of the effect of bars on light profiles, it is illustrative to first gauge the overall characteristics of each type of profile, regardless of the presence or absence of a bar. In the following subsections, we describe the global distribution of disk scale length, surface brightness, disk isophotal size, break radius, and stellar density at the break. As a guide, the main results are schematically summarized in Figure 5, which presents a simple schematic comparison of a single-exponential profile and a down-bending one for galaxies of the same total stellar mass.

\subsubsection{Disk Scale Length}

In Figure 6, we plot the disk scale length and central surface brightness of Type II profiles (for both the inner and outer disks) 


\begin{tabular}{|c|c|c|c|c|c|c|c|c|c|c|c|c|c|c|c|c|}
\hline \multirow[b]{2}{*}{ Galaxy } & \multirow{2}{*}{$\begin{array}{l}\text { Bar } \\
\text { Flag }\end{array}$} & \multicolumn{3}{|c|}{$\epsilon_{\max }$} & \multicolumn{3}{|c|}{$\epsilon_{\min }$} & \multicolumn{3}{|c|}{$\Delta \mathrm{P} . \mathrm{A} .=10^{\circ}$} & \multicolumn{3}{|c|}{$\Delta \epsilon=0.1$} & \multicolumn{3}{|c|}{ Final Deprojected } \\
\hline & & $a\left({ }^{\prime \prime}\right)$ & $\epsilon$ & P.A. (deg) & $a\left({ }^{\prime \prime}\right)$ & $\epsilon$ & P.A. (deg) & $a\left({ }^{\prime \prime}\right)$ & $\epsilon$ & P.A. (deg) & $a\left({ }^{\prime \prime}\right)$ & $\epsilon$ & P.A. (deg) & $a\left({ }^{\prime \prime}\right)$ & $\epsilon$ & P.A. (deg) \\
\hline ESO026-001 & C & 6 & 0.534 & 66.3 & 16 & 0.478 & 61.7 & 20.2 & 0.596 & 56.3 & 26.4 & 0.434 & 22.1 & $11.5 \pm 5.2$ & 0.542 & 35.6 \\
\hline ESO027-001 & $\mathrm{B}$ & 18 & 0.533 & 59.4 & 28 & 0.285 & 102.6 & 23.2 & 0.464 & 69.4 & 24.4 & 0.433 & 74.3 & $26.9 \pm 3.7$ & 0.628 & 165.4 \\
\hline ESO079-007 & $\mathrm{B}$ & 10 & 0.628 & 24.3 & 16 & 0.150 & 5.6 & 13.7 & 0.350 & 14.3 & 12.4 & 0.528 & 24.7 & $11.2 \pm 1.2$ & 0.547 & 6.1 \\
\hline ESO482-035 & B & 20 & 0.723 & 28.5 & 26 & 0.444 & 18.0 & 25.8 & 0.447 & 18.5 & 22.6 & 0.623 & 27.1 & $24.6 \pm 1.5$ & 0.673 & 41.9 \\
\hline ESO549-018 & $\mathrm{C}$ & 20 & 0.656 & 16.5 & 26 & 0.169 & 39.2 & 24.7 & 0.172 & 26.5 & 22.3 & 0.556 & 18.6 & $21.2 \pm 1.2$ & 0.442 & 3.2 \\
\hline IC0167 & $\mathrm{B}$ & 6 & 0.487 & 137.5 & 8 & 0.478 & 130.7 & 9.1 & 0.503 & 127.5 & 16.6 & 0.387 & 93.6 & $8.7 \pm 1.0$ & 0.430 & 147.3 \\
\hline IC0749 & $\mathrm{C}$ & 10 & 0.586 & 40.8 & 16 & 0.139 & 23.5 & 15.2 & 0.273 & 30.8 & 13.6 & 0.486 & 41.7 & $13.2 \pm 2.0$ & 0.630 & 2.8 \\
\hline IC0800 & B & 20 & 0.779 & 149.0 & 38 & 0.269 & 155.3 & 55.0 & 0.291 & 159.0 & 23.9 & 0.679 & 155.0 & $22.1 \pm 1.9$ & 0.721 & 165.5 \\
\hline IC1158 & $\mathrm{C}$ & 4 & 0.364 & 76.9 & 6 & 0.060 & 133.4 & 4.3 & 0.310 & 86.9 & 4.7 & 0.264 & 95.5 & $6.5 \pm 0.1$ & 0.561 & 14.4 \\
\hline IC1933 & $\mathrm{C}$ & 12 & 0.639 & 47.3 & 24 & 0.385 & 62.0 & 17.6 & 0.583 & 57.3 & 18.6 & 0.539 & 63.4 & $15.0 \pm 2.7$ & 0.392 & 163.5 \\
\hline IC1954 & B & 4 & 0.698 & 90.1 & 10 & 0.338 & 110.9 & 9.0 & 0.436 & 100.1 & 6.8 & 0.598 & 89.0 & $6.8 \pm 1.8$ & 0.658 & 139.0 \\
\hline IC2007 & B & 10 & 0.744 & 14.1 & 16 & 0.309 & 31.4 & 14.1 & 0.453 & 24.1 & 12.0 & 0.644 & 14.4 & $14.2 \pm 1.4$ & 0.751 & 35.7 \\
\hline IC2051 & B & 22 & 0.472 & 13.9 & 28 & 0.280 & 44.8 & 25.3 & 0.353 & 23.9 & 25.1 & 0.372 & 22.4 & $36.3 \pm 1.8$ & 0.633 & 15.9 \\
\hline IC4237 & B & 16 & 0.691 & 117.5 & 20 & 0.146 & 127.4 & 16.7 & 0.549 & 127.5 & 16.5 & 0.591 & 124.6 & $16.9 \pm 0.1$ & 0.626 & 151.5 \\
\hline IC5069 & B & 12 & 0.539 & 51.0 & 24 & 0.034 & 31.5 & 16.9 & 0.435 & 61.0 & 16.9 & 0.439 & 60.7 & $14.6 \pm 2.5$ & 0.535 & 35.4 \\
\hline IC5332 & $\mathrm{C}$ & 6 & 0.374 & 128.2 & 14 & 0.138 & 37.0 & 8.0 & 0.316 & 118.2 & 10.9 & 0.274 & 94.2 & $7.3 \pm 1.0$ & 0.399 & 160.4 \\
\hline NGC 0150 & B & 18 & 0.616 & 54.1 & 26 & 0.207 & 170.5 & 22.2 & 0.510 & 44.1 & 22.1 & 0.516 & 44.6 & $35.1 \pm 4.8$ & 0.740 & 18.2 \\
\hline NGC 0254 & B & 26 & 0.510 & 132.1 & 38 & 0.467 & 133.2 & $\ldots$ & $\ldots$ & $\ldots$ & 57.8 & 0.410 & 129.1 & $32.0 \pm 6.0$ & 0.249 & 1.1 \\
\hline NGC 0255 & B & 4 & 0.676 & 57.0 & 10 & 0.492 & 56.6 & 15.9 & 0.423 & 47.0 & 7.9 & 0.576 & 55.2 & $6.2 \pm 2.0$ & 0.677 & 137.9 \\
\hline NGC 0289 & B & 18 & 0.578 & 125.2 & 20 & 0.000 & 174.1 & 18.4 & 0.460 & 135.2 & 18.4 & 0.478 & 133.6 & $18.4 \pm 0.4$ & 0.475 & 9.3 \\
\hline NGC 0337 & $\mathrm{C}$ & 14 & 0.767 & 1.0 & 24 & 0.654 & 176.0 & 27.5 & 0.666 & 171.0 & 23.5 & 0.667 & 176.0 & $24.4 \pm 5.9$ & 0.811 & 157.6 \\
\hline NGC 0470 & B & 24 & 0.550 & 11.3 & 30 & 0.225 & 170.1 & 28.2 & 0.267 & 1.3 & 25.4 & 0.450 & 11.1 & $37.6 \pm 1.6$ & 0.616 & 154.5 \\
\hline NGC 0685 & B & 20 & 0.716 & 54.9 & 30 & 0.167 & 169.0 & 26.5 & 0.473 & 44.9 & 22.2 & 0.616 & 57.7 & $24.6 \pm 1.1$ & 0.731 & 34.9 \\
\hline NGC 0718 & B & 20 & 0.414 & 153.9 & 28 & 0.317 & 149.3 & 30.8 & 0.312 & 143.9 & 30.4 & 0.314 & 145.2 & $25.1 \pm 4.3$ & 0.388 & 142.6 \\
\hline NGC 0918 & $\mathrm{C}$ & 6 & 0.422 & 6.9 & 10 & 0.413 & 167.0 & 8.5 & 0.422 & 176.9 & 15.0 & 0.322 & 150.7 & $9.1 \pm 1.2$ & 0.434 & 151.5 \\
\hline NGC 0936 & B & 40 & 0.489 & 80.6 & 58 & 0.160 & 112.2 & 50.7 & 0.270 & 90.6 & 48.2 & 0.389 & 83.1 & $52.4 \pm 4.8$ & 0.521 & 31.9 \\
\hline NGC 0941 & $\mathrm{C}$ & 10 & 0.472 & 13.4 & 14 & 0.343 & 35.7 & 11.2 & 0.441 & 23.4 & 13.2 & 0.372 & 33.4 & $12.3 \pm 1.1$ & 0.418 & 135.1 \\
\hline NGC 0986 & B & 48 & 0.691 & 56.9 & 74 & 0.546 & 38.0 & 64.6 & 0.593 & 46.9 & 64.6 & 0.591 & 46.8 & $61.6 \pm 9.5$ & 0.707 & 28.9 \\
\hline NGC 0991 & $\mathrm{C}$ & 24 & 0.419 & 83.7 & 28 & 0.234 & 71.0 & 24.6 & 0.378 & 73.7 & 25.5 & 0.319 & 59.0 & $25.4 \pm 0.4$ & 0.432 & 31.9 \\
\hline NGC 1015 & B & 22 & 0.527 & 102.1 & 26 & 0.072 & 103.1 & 27.0 & 0.072 & 92.1 & 24.4 & 0.427 & 102.0 & $24.2 \pm 1.3$ & 0.537 & 32.9 \\
\hline NGC 1022 & B & 18 & 0.401 & 107.3 & 34 & 0.145 & 58.7 & 22.8 & 0.365 & 97.3 & 26.1 & 0.301 & 89.4 & $22.1 \pm 2.5$ & 0.443 & 160.8 \\
\hline NGC 1042 & $\mathrm{C}$ & 32 & 0.648 & 115.9 & 44 & 0.341 & 79.9 & 40.2 & 0.625 & 105.9 & 42.4 & 0.548 & 97.9 & $45.2 \pm 5.5$ & 0.709 & 16.1 \\
\hline NGC 1073 & B & 46 & 0.731 & 60.6 & 68 & 0.079 & 144.6 & 66.1 & 0.488 & 50.6 & 60.8 & 0.631 & 62.7 & $58.4 \pm 8.2$ & 0.735 & 140.6 \\
\hline NGC 1087 & B & 18 & 0.561 & 137.2 & 22 & 0.178 & 79.6 & 18.6 & 0.462 & 127.2 & 18.6 & 0.461 & 127.1 & $25.4 \pm 1.3$ & 0.611 & 28.6 \\
\hline NGC 1097 & B & 94 & 0.648 & 147.0 & 118 & 0.508 & 144.6 & 170.5 & 0.453 & 157.0 & 115.9 & 0.548 & 147.9 & $110.0 \pm 12.1$ & 0.515 & 24.0 \\
\hline NGC 1179 & B & 14 & 0.534 & 155.7 & 20 & 0.203 & 166.5 & 17.5 & 0.324 & 165.7 & 15.1 & 0.434 & 151.8 & $18.4 \pm 0.8$ & 0.621 & 14.3 \\
\hline NGC 1187 & $\mathrm{C}$ & 40 & 0.730 & 127.9 & 42 & 0.462 & 118.7 & 42.8 & 0.469 & 117.9 & 40.8 & 0.630 & 124.5 & $40.4 \pm 0.4$ & 0.649 & 4.1 \\
\hline NGC 1232 & B & 24 & 0.560 & 89.3 & 36 & 0.067 & 52.5 & 29.2 & 0.427 & 99.3 & 27.9 & 0.460 & 95.4 & $26.0 \pm 1.9$ & 0.483 & 171.3 \\
\hline NGC 1255 & $\mathrm{C}$ & 6 & 0.580 & 117.2 & 12 & 0.449 & 115.9 & 32.9 & 0.484 & 107.2 & 10.0 & 0.480 & 115.8 & $8.1 \pm 2.1$ & 0.431 & 169.2 \\
\hline NGC 1258 & $\mathrm{C}$ & 10 & 0.341 & 80.2 & 14 & 0.221 & 50.4 & 12.0 & 0.256 & 70.2 & 12.8 & 0.241 & 62.2 & $14.2 \pm 1.1$ & 0.478 & 165.5 \\
\hline
\end{tabular}




\begin{tabular}{|c|c|c|c|c|c|c|c|c|c|c|c|c|c|c|c|c|}
\hline \multirow[b]{2}{*}{ Galaxy } & \multirow{2}{*}{$\begin{array}{l}\text { Bar } \\
\text { Flag }\end{array}$} & \multicolumn{3}{|c|}{$\epsilon_{\max }$} & \multicolumn{3}{|c|}{$\epsilon_{\min }$} & \multicolumn{3}{|c|}{$\Delta \mathrm{P} . \mathrm{A} .=10^{\circ}$} & \multicolumn{3}{|c|}{$\Delta \epsilon=0.1$} & \multicolumn{3}{|c|}{ Final Deprojected } \\
\hline & & $a\left({ }^{\prime \prime}\right)$ & $\epsilon$ & P.A. (deg) & $a\left({ }^{\prime \prime}\right)$ & $\epsilon$ & P.A. (deg) & $a\left({ }^{\prime \prime}\right)$ & $\epsilon$ & P.A. (deg) & $a(")$ & $\epsilon$ & P.A. (deg) & $a\left(^{\prime \prime}\right)$ & $\epsilon$ & P.A. (deg) \\
\hline NGC 1300 & $\mathrm{~B}$ & 90 & 0.786 & 102.0 & 134 & 0.389 & 89.4 & 125.7 & 0.447 & 92.0 & 124.3 & 0.686 & 105.4 & $111.4 \pm 17.5$ & 0.781 & 139.6 \\
\hline NGC 1302 & $\mathrm{~B}$ & 28 & 0.339 & 169.5 & 42 & 0.140 & 177.4 & 62.7 & 0.125 & 159.5 & 33.3 & 0.239 & 176.9 & $30.7 \pm 2.6$ & 0.292 & 172.2 \\
\hline NGC 1306 & $\mathrm{C}$ & 8 & 0.433 & 100.8 & 18 & 0.063 & 49.4 & 13.5 & 0.235 & 90.8 & 11.1 & 0.333 & 96.6 & $11.3 \pm 1.9$ & 0.474 & 29.8 \\
\hline NGC $1325 \mathrm{~A}$ & $\mathrm{C}$ & 22 & 0.404 & 175.5 & 26 & 0.012 & 132.5 & 24.7 & 0.256 & 165.5 & 24.5 & 0.304 & 172.0 & $24.8 \pm 1.4$ & 0.415 & 36.8 \\
\hline NGC 1326 & B & 32 & 0.423 & 18.3 & 44 & 0.246 & 24.5 & 70.4 & 0.153 & 28.3 & 37.4 & 0.323 & 22.0 & $42.8 \pm 3.2$ & 0.491 & 25.8 \\
\hline NGC 1338 & B & 12 & 0.545 & 121.2 & 16 & 0.136 & 116.3 & 13.0 & 0.458 & 111.2 & 13.1 & 0.445 & 109.7 & $13.9 \pm 0.4$ & 0.583 & 156.8 \\
\hline NGC 1341 & $\mathrm{~B}$ & 10 & 0.672 & 161.4 & 22 & 0.393 & 121.6 & 16.6 & 0.560 & 151.4 & 16.4 & 0.572 & 152.5 & $13.3 \pm 3.3$ & 0.653 & 169.8 \\
\hline NGC 1350 & B & 56 & 0.582 & 37.5 & 62 & 0.502 & 34.5 & 68.5 & 0.512 & 27.5 & 127.0 & 0.482 & 23.9 & $81.6 \pm 3.9$ & 0.586 & 148.5 \\
\hline NGC 1367 & $\mathrm{C}$ & 22 & 0.447 & 107.6 & 34 & 0.222 & 116.0 & 29.1 & 0.363 & 97.6 & 29.8 & 0.347 & 96.5 & $31.1 \pm 5.6$ & 0.379 & 41.1 \\
\hline NGC 1398 & $\mathrm{~B}$ & 40 & 0.381 & 13.2 & 46 & 0.007 & 177.2 & 45.5 & 0.072 & 3.2 & 43.4 & 0.281 & 17.2 & $54.7 \pm 2.1$ & 0.528 & 4.5 \\
\hline NGC 1433 & $\mathrm{~B}$ & 86 & 0.656 & 95.1 & 124 & 0.294 & 99.4 & 160.2 & 0.263 & 105.1 & 104.6 & 0.556 & 97.4 & $110.2 \pm 11.0$ & 0.696 & 161.5 \\
\hline NGC 1436 & $\mathrm{C}$ & 8 & 0.319 & 170.1 & 12 & 0.235 & 133.3 & 10.6 & 0.265 & 160.1 & 149.0 & 0.219 & 150.0 & $10.3 \pm 1.3$ & 0.260 & 142.7 \\
\hline NGC 1438 & B & 28 & 0.715 & 54.3 & 48 & 0.537 & 67.9 & 39.4 & 0.579 & 64.3 & 35.4 & 0.615 & 59.2 & $34.9 \pm 3.4$ & 0.571 & 145.1 \\
\hline NGC 1452 & B & 26 & 0.489 & 31.4 & 36 & 0.082 & 24.7 & 36.4 & 0.082 & 41.4 & 34.2 & 0.389 & 25.8 & $47.3 \pm 6.6$ & 0.673 & 4.0 \\
\hline NGC 1493 & B & 26 & 0.620 & 78.8 & 44 & 0.013 & 2.7 & 36.6 & 0.304 & 68.8 & 30.2 & 0.520 & 76.9 & $28.3 \pm 2.2$ & 0.593 & 162.3 \\
\hline NGC 1512 & $\mathrm{~B}$ & 72 & 0.661 & 43.5 & 98 & 0.291 & 46.1 & 178.5 & 0.327 & 53.5 & 88.4 & 0.561 & 45.2 & $88.7 \pm 8.9$ & 0.626 & 139.0 \\
\hline NGC 1533 & $\mathrm{~B}$ & 24 & 0.372 & 166.4 & 42 & 0.065 & 109.9 & 35.1 & 0.189 & 156.4 & 32.6 & 0.272 & 162.7 & $28.5 \pm 4.3$ & 0.361 & 29.4 \\
\hline NGC 1559 & B & 8 & 0.780 & 84.5 & 24 & 0.420 & 74.6 & 24.1 & 0.426 & 74.5 & 13.6 & 0.680 & 79.1 & $11.4 \pm 2.8$ & 0.694 & 30.3 \\
\hline NGC 1566 & $\mathrm{~B}$ & 32 & 0.509 & 3.1 & 44 & 0.129 & 94.5 & 37.2 & 0.358 & 13.1 & 36.8 & 0.409 & 10.1 & $40.5 \pm 2.8$ & 0.584 & 1.7 \\
\hline NGC 1637 & $\mathrm{~B}$ & 22 & 0.521 & 77.0 & 38 & 0.132 & 65.9 & 26.8 & 0.481 & 67.0 & 30.5 & 0.421 & 52.1 & $30.6 \pm 2.3$ & 0.598 & 156.8 \\
\hline NGC 1640 & $\mathrm{~B}$ & 30 & 0.631 & 46.0 & 38 & 0.303 & 47.3 & 54.5 & 0.055 & 56.0 & 33.9 & 0.531 & 47.1 & $32.0 \pm 1.9$ & 0.589 & 171.0 \\
\hline NGC 1672 & $\mathrm{~B}$ & 84 & 0.695 & 94.6 & 92 & 0.519 & 94.3 & 109.9 & 0.519 & 84.6 & 91.0 & 0.595 & 93.5 & $95.6 \pm 3.9$ & 0.711 & 29.5 \\
\hline NGC 1688 & $\mathrm{~B}$ & 10 & 0.755 & 137.9 & 64 & 0.287 & 170.3 & 40.7 & 0.600 & 147.9 & 38.9 & 0.655 & 145.3 & $30.0 \pm 17.2$ & 0.767 & 34.7 \\
\hline NGC 1808 & B & 76 & 0.672 & 144.4 & 136 & 0.572 & 140.7 & 234.8 & 0.600 & 134.4 & 161.6 & 0.572 & 138.4 & $114.0 \pm 31.5$ & 0.615 & 35.6 \\
\hline NGC 2500 & $\mathrm{C}$ & 6 & 0.450 & 6.7 & 12 & 0.357 & 13.7 & 8.6 & 0.428 & 16.7 & 34.8 & 0.350 & 32.1 & $7.9 \pm 1.3$ & 0.476 & 31.5 \\
\hline NGC 2633 & B & 20 & 0.584 & 170.8 & 24 & 0.523 & 171.1 & 32.8 & 0.562 & 160.8 & 36.2 & 0.484 & 155.9 & $22.8 \pm 2.1$ & 0.449 & 157.0 \\
\hline NGC 3049 & B & 42 & 0.792 & 30.2 & 52 & 0.376 & 28.7 & 87.7 & 0.202 & 40.2 & 48.4 & 0.692 & 27.6 & $45.2 \pm 3.2$ & 0.690 & 2.2 \\
\hline NGC 3184 & $\mathrm{C}$ & 24 & 0.323 & 71.1 & 32 & 0.163 & 124.8 & 26.6 & 0.246 & 81.1 & 28.2 & 0.223 & 97.0 & $27.2 \pm 1.2$ & 0.366 & 20.5 \\
\hline NGC 3206 & $\mathrm{C}$ & 6 & 0.612 & 80.3 & 14 & 0.381 & 85.0 & 21.9 & 0.240 & 90.3 & 7.2 & 0.512 & 83.0 & $9.0 \pm 0.8$ & 0.710 & 168.4 \\
\hline NGC 3319 & B & 18 & 0.861 & 38.7 & 30 & 0.776 & 38.6 & 75.5 & 0.599 & 28.7 & 40.1 & 0.761 & 37.6 & $24.1 \pm 6.0$ & 0.758 & 172.9 \\
\hline NGC 3344 & $\mathrm{~B}$ & 26 & 0.379 & 6.6 & 32 & 0.098 & 158.1 & 27.6 & 0.310 & 16.6 & 28.2 & 0.279 & 17.7 & $29.4 \pm 1.0$ & 0.423 & 160.1 \\
\hline NGC 3351 & B & 54 & 0.465 & 112.5 & 68 & 0.119 & 157.1 & 64.7 & 0.349 & 122.5 & 64.6 & 0.365 & 121.1 & $79.0 \pm 6.5$ & 0.599 & 6.9 \\
\hline NGC 3368 & B & 64 & 0.420 & 125.6 & 92 & 0.340 & 142.3 & 74.9 & 0.374 & 135.6 & 142.3 & 0.320 & 135.0 & $96.0 \pm 5.0$ & 0.534 & 21.5 \\
\hline NGC 3486 & B & 22 & 0.558 & 75.5 & 28 & 0.225 & 72.4 & 33.4 & 0.249 & 65.5 & 22.8 & 0.458 & 76.3 & $22.8 \pm 0.4$ & 0.410 & 163.1 \\
\hline NGC 3504 & $\mathrm{~B}$ & 34 & 0.651 & 145.8 & 44 & 0.499 & 150.6 & 58.9 & 0.507 & 155.8 & 38.6 & 0.551 & 149.2 & $36.4 \pm 2.3$ & 0.627 & 8.2 \\
\hline NGC 3627 & B & 62 & 0.767 & 159.6 & 82 & 0.272 & 172.1 & 81.0 & 0.278 & 169.6 & 75.3 & 0.667 & 155.9 & $75.5 \pm 9.2$ & 0.625 & 153.5 \\
\hline NGC 3726 & B & 42 & 0.786 & 30.4 & 46 & 0.486 & 26.5 & 51.2 & 0.530 & 20.4 & 42.7 & 0.686 & 30.4 & $43.8 \pm 0.4$ & 0.672 & 18.9 \\
\hline NGC 3953 & B & 28 & 0.544 & 47.4 & 38 & 0.369 & 39.4 & 38.6 & 0.376 & 37.4 & 36.3 & 0.444 & 47.9 & $47.7 \pm 7.0$ & 0.583 & 152.4 \\
\hline NGC 4051 & B & 66 & 0.745 & 131.6 & 82 & 0.587 & 133.9 & 102.1 & 0.550 & 121.6 & 73.4 & 0.645 & 138.4 & $70.6 \pm 4.3$ & 0.665 & 8.8 \\
\hline NGC 4136 & $\mathrm{C}$ & 16 & 0.503 & 22.8 & 20 & 0.140 & 90.3 & 18.4 & 0.425 & 32.8 & 18.6 & 0.403 & 37.2 & $18.2 \pm 1.3$ & 0.524 & 158.7 \\
\hline
\end{tabular}




\begin{tabular}{|c|c|c|c|c|c|c|c|c|c|c|c|c|c|c|c|c|}
\hline \multirow[b]{2}{*}{ Galaxy } & \multirow{2}{*}{$\begin{array}{l}\text { Bar } \\
\text { Flag }\end{array}$} & \multicolumn{3}{|c|}{$\epsilon_{\max }$} & \multicolumn{3}{|c|}{$\epsilon_{\min }$} & \multicolumn{3}{|c|}{$\Delta \mathrm{P} . \mathrm{A} .=10^{\circ}$} & \multicolumn{3}{|c|}{$\Delta \epsilon=0.1$} & \multicolumn{3}{|c|}{ Final Deprojected } \\
\hline & & $a\left({ }^{\prime \prime}\right)$ & $\epsilon$ & P.A. (deg) & $a\left({ }^{\prime \prime}\right)$ & $\epsilon$ & P.A. (deg) & $a(")$ & $\epsilon$ & P.A. (deg) & $a\left({ }^{\prime \prime}\right)$ & $\epsilon$ & P.A. (deg) & $a(")$ & $\epsilon$ & P.A. (deg) \\
\hline NGC 4245 & B & 36 & 0.521 & 135.1 & 46 & 0.252 & 145.6 & 43.2 & 0.327 & 145.1 & 40.7 & 0.421 & 140.4 & $42.5 \pm 2.3$ & 0.535 & 36.2 \\
\hline NGC 4262 & B & 14 & 0.300 & 17.4 & 20 & 0.118 & 164.2 & 16.8 & 0.175 & 7.4 & 16.2 & 0.200 & 10.8 & $15.4 \pm 1.1$ & 0.258 & 30.7 \\
\hline NGC 4303 & B & 52 & 0.695 & 4.9 & 60 & 0.203 & 11.2 & 66.3 & 0.259 & 14.9 & 58.3 & 0.595 & 5.1 & $56.8 \pm 3.3$ & 0.683 & 35.0 \\
\hline NGC 4314 & B & 66 & 0.647 & 146.7 & 102 & 0.416 & 145.9 & 106.5 & 0.354 & 136.7 & 88.4 & 0.547 & 147.1 & $83.4 \pm 12.1$ & 0.672 & 12.7 \\
\hline NGC 4319 & B & 18 & 0.510 & 162.4 & 22 & 0.221 & 159.3 & 22.9 & 0.221 & 152.4 & 20.4 & 0.410 & 165.4 & $20.1 \pm 1.4$ & 0.424 & 27.8 \\
\hline NGC 4321 & $\mathrm{C}$ & 60 & 0.608 & 104.8 & 72 & 0.169 & 135.7 & 70.6 & 0.458 & 114.8 & 70.4 & 0.508 & 111.2 & $80.5 \pm 5.0$ & 0.679 & 16.9 \\
\hline NGC 4369 & B & 4 & 0.598 & 152.2 & 14 & 0.071 & 160.3 & 11.5 & 0.187 & 162.2 & 8.2 & 0.498 & 149.5 & $6.2 \pm 2.2$ & 0.606 & 167.8 \\
\hline NGC 4394 & B & 42 & 0.581 & 142.7 & 54 & 0.221 & 140.6 & 80.4 & 0.088 & 132.7 & 49.6 & 0.481 & 144.3 & $49.8 \pm 4.3$ & 0.571 & 44.9 \\
\hline NGC 4405 & B & 10 & 0.596 & 7.6 & 18 & 0.276 & 15.2 & 30.1 & 0.320 & 17.6 & 11.2 & 0.496 & 11.5 & $10.7 \pm 0.6$ & 0.470 & 166.3 \\
\hline NGC 4413 & B & 8 & 0.580 & 14.0 & 22 & 0.096 & 13.1 & 22.7 & 0.097 & 24.0 & 19.0 & 0.480 & 13.8 & $17.7 \pm 7.3$ & 0.643 & 25.9 \\
\hline NGC 4424 & B & 8 & 0.840 & 110.3 & 18 & 0.616 & 104.5 & 36.0 & 0.616 & 100.3 & 11.7 & 0.740 & 109.1 & $11.7 \pm 2.2$ & 0.785 & 40.1 \\
\hline NGC 4450 & $\mathrm{C}$ & 44 & 0.542 & 7.4 & 54 & 0.404 & 4.1 & 101.8 & 0.431 & 177.4 & 49.6 & 0.442 & 8.6 & $49.4 \pm 3.4$ & 0.393 & 27.0 \\
\hline NGC 4457 & B & 30 & 0.378 & 66.3 & 50 & 0.281 & 73.8 & 73.0 & 0.412 & 76.3 & 82.6 & 0.278 & 76.2 & $40.0 \pm 10.0$ & 0.347 & 176.1 \\
\hline NGC 4498 & B & 32 & 0.813 & 123.1 & 48 & 0.541 & 131.0 & 56.6 & 0.560 & 133.1 & 40.2 & 0.713 & 122.1 & $40.3 \pm 5.0$ & 0.718 & 148.9 \\
\hline NGC 4548 & B & 58 & 0.538 & 61.0 & 70 & 0.402 & 66.3 & 73.4 & 0.400 & 71.0 & 68.5 & 0.438 & 63.7 & $73.9 \pm 6.1$ & 0.605 & 5.4 \\
\hline NGC 4579 & B & 42 & 0.486 & 58.9 & 54 & 0.319 & 70.4 & 53.6 & 0.328 & 68.9 & 51.0 & 0.386 & 62.6 & $54.3 \pm 5.0$ & 0.488 & 36.8 \\
\hline NGC 4639 & B & 24 & 0.562 & 168.7 & 48 & 0.178 & 140.0 & 34.1 & 0.303 & 158.7 & 30.6 & 0.462 & 163.3 & $34.9 \pm 3.8$ & 0.592 & 148.5 \\
\hline NGC 4713 & $\mathrm{C}$ & 12 & 0.636 & 83.9 & 16 & 0.077 & 38.9 & 14.2 & 0.496 & 73.9 & 14.1 & 0.536 & 77.3 & $13.6 \pm 1.3$ & 0.534 & 159.0 \\
\hline NGC 4725 & B & 128 & 0.685 & 49.0 & 166 & 0.332 & 42.4 & 175.4 & 0.349 & 39.0 & 148.0 & 0.585 & 49.1 & $144.8 \pm 11.0$ & 0.540 & 23.5 \\
\hline NGC 4942 & $\mathrm{C}$ & 6 & 0.387 & 174.1 & 8 & 0.181 & 170.7 & 8.9 & 0.181 & 4.1 & 7.0 & 0.287 & 172.5 & $6.9 \pm 0.5$ & 0.338 & 40.5 \\
\hline NGC 4984 & B & 28 & 0.305 & 92.2 & 52 & 0.122 & 74.2 & 37.3 & 0.202 & 82.2 & 37.2 & 0.205 & 82.4 & $50.8 \pm 6.8$ & 0.538 & 169.5 \\
\hline NGC 5068 & B & 20 & 0.728 & 149.1 & 32 & 0.509 & 151.4 & 41.9 & 0.472 & 159.1 & 27.7 & 0.628 & 151.4 & $24.0 \pm 3.9$ & 0.728 & 44.9 \\
\hline NGC 5339 & B & 24 & 0.702 & 81.9 & 38 & 0.038 & 53.6 & 34.6 & 0.402 & 71.9 & 31.0 & 0.602 & 83.7 & $31.8 \pm 4.2$ & 0.716 & 144.7 \\
\hline NGC 5371 & B & 22 & 0.394 & 98.4 & 36 & 0.357 & 74.8 & 28.4 & 0.370 & 88.4 & 55.9 & 0.294 & 14.1 & $31.4 \pm 3.8$ & 0.515 & 173.9 \\
\hline NGC 5377 & B & 58 & 0.661 & 44.8 & 72 & 0.625 & 42.0 & 114.1 & 0.431 & 34.8 & 112.6 & 0.561 & 43.5 & $72.9 \pm 7.0$ & 0.518 & 38.1 \\
\hline NGC 5468 & $\mathrm{C}$ & 8 & 0.420 & 146.9 & 12 & 0.309 & 166.4 & 10.3 & 0.358 & 156.9 & 11.6 & 0.320 & 164.3 & $9.6 \pm 1.3$ & 0.434 & 146.9 \\
\hline NGC 5584 & B & 8 & 0.490 & 36.6 & 12 & 0.333 & 25.5 & 11.8 & 0.350 & 26.6 & 11.2 & 0.390 & 29.3 & $12.0 \pm 1.9$ & 0.570 & 157.8 \\
\hline NGC 5597 & B & 14 & 0.535 & 49.5 & 32 & 0.428 & 84.2 & 25.1 & 0.540 & 59.5 & 29.5 & 0.435 & 73.8 & $26.6 \pm 7.3$ & 0.661 & 6.1 \\
\hline NGC 5645 & $\mathrm{C}$ & 12 & 0.554 & 133.8 & 18 & 0.333 & 112.3 & 15.0 & 0.449 & 123.8 & 14.9 & 0.454 & 124.3 & $19.1 \pm 1.7$ & 0.674 & 164.1 \\
\hline NGC 5668 & $\mathrm{C}$ & 10 & 0.310 & 111.7 & 14 & 0.097 & 129.7 & 11.7 & 0.315 & 101.7 & 13.0 & 0.210 & 114.3 & $11.5 \pm 1.0$ & 0.263 & 141.2 \\
\hline NGC 5669 & B & 6 & 0.751 & 40.5 & 16 & 0.025 & 162.5 & 12.1 & 0.611 & 50.5 & 11.4 & 0.651 & 46.4 & $8.9 \pm 2.7$ & 0.694 & 154.4 \\
\hline NGC 5713 & $\mathrm{C}$ & 20 & 0.634 & 98.3 & 30 & 0.157 & 128.3 & 22.8 & 0.423 & 88.3 & 21.7 & 0.534 & 93.3 & $23.9 \pm 0.9$ & 0.681 & 174.5 \\
\hline NGC 5740 & B & 16 & 0.472 & 128.4 & 22 & 0.411 & 145.9 & 20.6 & 0.446 & 138.4 & $\ldots$ & $\ldots$ & $\ldots$ & $24.7 \pm 2.1$ & 0.518 & 26.5 \\
\hline NGC 5850 & B & 62 & 0.628 & 116.0 & 80 & 0.259 & 124.4 & 99.2 & 0.356 & 126.0 & 77.6 & 0.528 & 118.5 & $78.9 \pm 8.6$ & 0.626 & 40.8 \\
\hline NGC 5892 & B & 4 & 0.426 & 158.5 & 8 & 0.372 & 156.0 & 12.7 & 0.325 & 168.5 & 12.6 & 0.326 & 167.7 & $6.6 \pm 2.2$ & 0.460 & 151.9 \\
\hline NGC 5950 & B & 4 & 0.678 & 91.7 & 10 & 0.195 & 178.7 & 6.3 & 0.556 & 101.7 & 6.2 & 0.578 & 99.8 & $8.2 \pm 2.0$ & 0.749 & 156.0 \\
\hline NGC 5958 & $\mathrm{C}$ & 6 & 0.505 & 135.0 & 8 & 0.265 & 141.5 & 8.4 & 0.296 & 145.0 & 6.8 & 0.405 & 137.7 & $7.6 \pm 0.5$ & 0.582 & 5.2 \\
\hline NGC 5964 & B & 18 & 0.830 & 148.4 & 32 & 0.725 & 147.9 & 54.9 & 0.581 & 138.4 & 30.6 & 0.730 & 147.8 & $24.3 \pm 6.3$ & 0.766 & 1.1 \\
\hline NGC 5985 & $\mathrm{C}$ & 10 & 0.394 & 32.5 & 12 & 0.387 & 28.0 & 14.5 & 0.419 & 22.5 & $\cdots$ & $\cdots$ & $\cdots$ & $14.5 \pm 1.1$ & 0.338 & 157.1 \\
\hline NGC 6012 & B & 20 & 0.715 & 155.6 & 30 & 0.606 & 152.0 & 50.5 & 0.352 & 165.6 & 28.1 & 0.615 & 152.1 & $26.6 \pm 4.7$ & 0.705 & 135.6 \\
\hline
\end{tabular}




\begin{tabular}{|c|c|c|c|c|c|c|c|c|c|c|c|c|c|c|c|c|}
\hline \multirow[b]{2}{*}{ Galaxy } & \multirow{2}{*}{$\begin{array}{l}\text { Bar } \\
\text { Flag }\end{array}$} & \multicolumn{3}{|c|}{$\epsilon_{\max }$} & \multicolumn{3}{|c|}{$\epsilon_{\min }$} & \multicolumn{3}{|c|}{$\Delta \mathrm{P} . \mathrm{A} .=10^{\circ}$} & \multicolumn{3}{|c|}{$\Delta \epsilon=0.1$} & \multicolumn{3}{|c|}{ Final Deprojected } \\
\hline & & $a\left({ }^{\prime \prime}\right)$ & $\epsilon$ & P.A. (deg) & $a\left({ }^{(\prime)}\right)$ & $\epsilon$ & P.A. (deg) & $a\left({ }^{\prime \prime}\right)$ & $\epsilon$ & P.A. (deg) & $a\left({ }^{\prime \prime}\right)$ & $\epsilon$ & P.A. (deg) & $a\left({ }^{\prime \prime}\right)$ & $\epsilon$ & P.A. (deg) \\
\hline NGC 6014 & B & 16 & 0.345 & 31.4 & 20 & 0.098 & 19.2 & 19.7 & 0.124 & 21.4 & 18.1 & 0.245 & 31.8 & $21.0 \pm 1.4$ & 0.457 & 166.6 \\
\hline NGC 6140 & B & 4 & 0.784 & 56.5 & 20 & 0.507 & 65.4 & 21.1 & 0.514 & 66.5 & 11.2 & 0.684 & 58.5 & $8.5 \pm 4.0$ & 0.796 & 32.7 \\
\hline NGC 6155 & $\mathrm{C}$ & 2 & 0.444 & 121.0 & 4 & 0.207 & 135.4 & 3.4 & 0.280 & 131.0 & 2.8 & 0.344 & 127.1 & $2.5 \pm 0.4$ & 0.343 & 152.5 \\
\hline NGC 6217 & B & 38 & 0.735 & 148.3 & 60 & 0.263 & 156.9 & 50.4 & 0.490 & 158.3 & 47.0 & 0.635 & 147.7 & $44.5 \pm 4.7$ & 0.746 & 164.1 \\
\hline NGC 6267 & B & 18 & 0.730 & 157.7 & 24 & 0.185 & 48.3 & 22.3 & 0.596 & 167.7 & 22.2 & 0.630 & 162.8 & $26.9 \pm 2.2$ & 0.784 & 23.1 \\
\hline NGC 6339 & B & 10 & 0.730 & 106.9 & 16 & 0.607 & 106.8 & 25.4 & 0.566 & 116.9 & 12.3 & 0.630 & 107.9 & $18.2 \pm 1.9$ & 0.832 & 7.6 \\
\hline NGC 6412 & B & 8 & 0.435 & 159.7 & 14 & 0.086 & 27.0 & 12.6 & 0.115 & 169.7 & 10.4 & 0.335 & 156.8 & $9.2 \pm 1.2$ & 0.432 & 15.4 \\
\hline NGC 6434 & B & 8 & 0.409 & 56.3 & 10 & 0.385 & 53.0 & 14.4 & 0.500 & 66.3 & $\ldots$ & $\ldots$ & $\ldots$ & $16.0 \pm 2.0$ & 0.578 & 16.7 \\
\hline NGC 6923 & $\mathrm{C}$ & 22 & 0.573 & 78.0 & 24 & 0.289 & 89.6 & 23.7 & 0.330 & 88.0 & 22.7 & 0.473 & 82.1 & $22.4 \pm 0.4$ & 0.349 & 0.1 \\
\hline NGC 7098 & $\mathrm{~B}$ & 42 & 0.550 & 50.2 & 58 & 0.455 & 55.8 & 67.0 & 0.491 & 60.2 & 110.1 & 0.450 & 63.1 & $58.1 \pm 8.7$ & 0.438 & 43.8 \\
\hline NGC 7140 & B & 58 & 0.754 & 18.8 & 82 & 0.474 & 22.4 & 89.7 & 0.386 & 28.8 & 66.2 & 0.654 & 19.1 & $65.4 \pm 4.6$ & 0.652 & 24.2 \\
\hline NGC 7479 & B & 50 & 0.757 & 7.8 & 82 & 0.618 & 22.1 & 79.0 & 0.678 & 17.8 & 79.5 & 0.657 & 18.7 & $64.8 \pm 14.2$ & 0.682 & 166.9 \\
\hline NGC 7552 & B & 36 & 0.656 & 89.6 & 64 & 0.486 & 97.9 & 66.7 & 0.490 & 99.6 & 59.7 & 0.556 & 96.6 & $49.4 \pm 12.3$ & 0.664 & 154.6 \\
\hline NGC 7661 & $\mathrm{C}$ & 8 & 0.687 & 48.1 & 10 & 0.428 & 38.2 & 22.2 & 0.484 & 38.1 & 8.8 & 0.587 & 44.3 & $8.9 \pm 0.3$ & 0.561 & 28.7 \\
\hline NGC 7731 & $\mathrm{C}$ & 8 & 0.439 & 42.8 & 12 & 0.347 & 45.3 & 20.3 & 0.340 & 52.8 & 20.3 & 0.339 & 52.9 & $12.2 \pm 2.4$ & 0.485 & 29.5 \\
\hline NGC 7741 & $\mathrm{~B}$ & 30 & 0.667 & 101.5 & 48 & 0.083 & 107.0 & 48.6 & 0.100 & 111.5 & 43.6 & 0.567 & 96.8 & $47.0 \pm 9.0$ & 0.729 & 16.8 \\
\hline NGC 7798 & $\mathrm{~B}$ & 10 & 0.485 & 94.5 & 14 & 0.164 & 149.5 & 12.6 & 0.360 & 104.5 & 12.4 & 0.385 & 98.8 & $12.3 \pm 1.4$ & 0.492 & 141.4 \\
\hline PGC003853 & $\mathrm{B}$ & 30 & 0.498 & 50.3 & 36 & 0.045 & 101.3 & 34.6 & 0.108 & 60.3 & 30.8 & 0.398 & 49.6 & $38.1 \pm 0.7$ & 0.567 & 24.6 \\
\hline PGC006667 & B & 16 & 0.461 & 53.2 & 24 & 0.090 & 109.7 & 20.9 & 0.223 & 63.2 & 19.6 & 0.361 & 46.0 & $22.7 \pm 2.4$ & 0.570 & 9.6 \\
\hline PGC011367 & $\mathrm{C}$ & 16 & 0.494 & 31.0 & 28 & 0.180 & 97.7 & 21.6 & 0.445 & 41.0 & 22.7 & 0.394 & 52.4 & $20.0 \pm 3.1$ & 0.497 & 138.9 \\
\hline PGC012633 & $\mathrm{C}$ & 18 & 0.510 & 101.6 & 22 & 0.195 & 105.4 & 23.7 & 0.196 & 111.6 & 19.2 & 0.410 & 99.8 & $18.8 \pm 0.6$ & 0.512 & 140.2 \\
\hline PGC012664 & B & 6 & 0.558 & 87.9 & 12 & 0.220 & 123.8 & 8.9 & 0.398 & 97.9 & 8.5 & 0.458 & 95.0 & $10.0 \pm 1.6$ & 0.674 & 13.7 \\
\hline PGC014037 & B & 12 & 0.660 & 142.5 & 18 & 0.511 & 136.9 & $\ldots$ & $\ldots$ & $\ldots$ & 15.5 & 0.560 & 139.5 & $13.8 \pm 1.7$ & 0.361 & 11.1 \\
\hline UGC00313 & $\mathrm{C}$ & 4 & 0.691 & 100.4 & 6 & 0.170 & 175.0 & 4.3 & 0.621 & 110.4 & 4.4 & 0.591 & 114.7 & $6.6 \pm 0.2$ & 0.807 & 177.3 \\
\hline UGC01551 & C & 20 & 0.656 & 80.7 & 30 & 0.128 & 127.3 & 24.3 & 0.447 & 90.7 & 22.7 & 0.556 & 79.9 & $23.0 \pm 1.6$ & 0.611 & 144.6 \\
\hline UGC09356 & $\mathrm{C}$ & 6 & 0.552 & 58.5 & 8 & 0.364 & 75.1 & 7.2 & 0.438 & 68.5 & 7.1 & 0.452 & 67.3 & $8.2 \pm 0.5$ & 0.571 & 32.8 \\
\hline
\end{tabular}

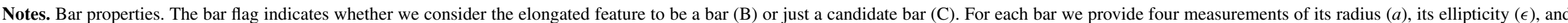

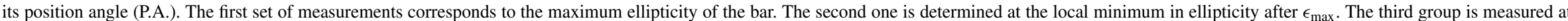

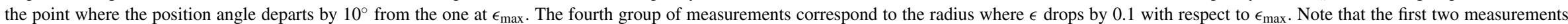

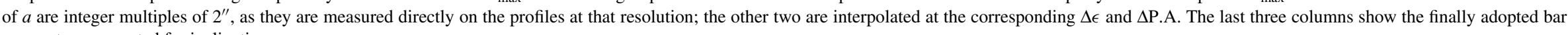
parameters, corrected for inclination.

(This table is also available in a machine-readable form in the online journal.) 


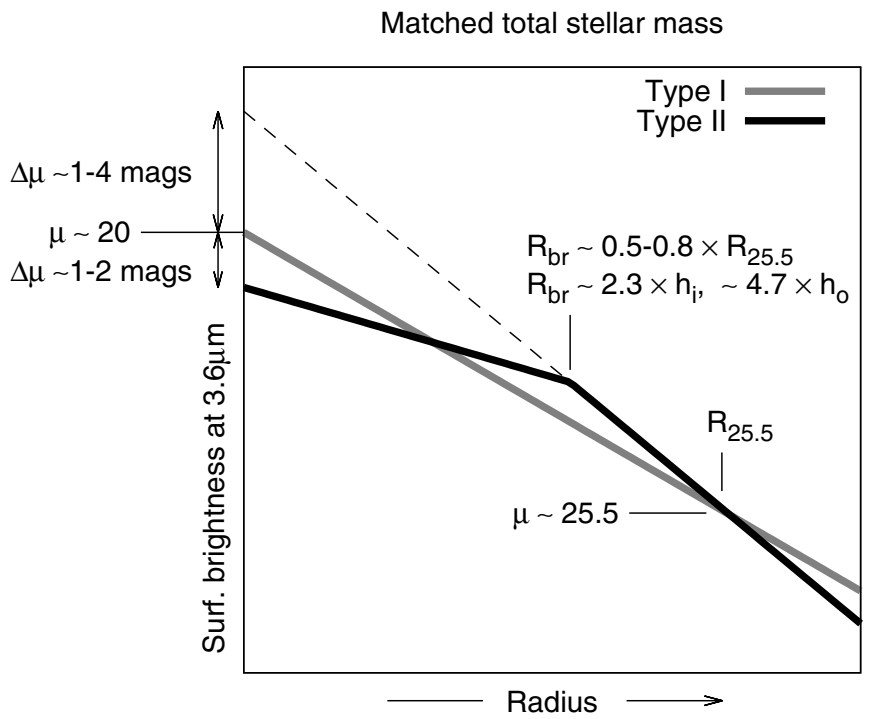

Figure 5. Schematic depiction of the structural properties of a down-bending disk, relative to a single-exponential one in a galaxy with the same total stellar mass. This plots summarizes the results of Figures 6-8. We show the break radius $R_{\text {br }}$ relative to the isophotal radius $R_{25.5}$ and the inner and outer scale lengths, $h_{i}$ and $h_{o}$. We also show the typical extrapolated central surface brightness of the inner and outer disks, relative to that of a single-exponential profile.

as a function of the absolute $3.6 \mu \mathrm{m}$ magnitude. As a reference, we compare these properties with those of Type I profiles. This allows us to establish a parallelism between single-exponential and down-bending profiles in galaxies with the same total stellar mass. ${ }^{25}$ The histograms to the right show the overall distribution of these structural parameters. In the particular case of the disk scale length, our measurements are consistent with the grid of simulations of disk breaks by Foyle et al. (2008), who found inner disk scale lengths in the range $1-10 \mathrm{kpc}$, and outer disk scale lengths between 0.5 and $5 \mathrm{kpc}$.

Panel (a) shows a clear correlation between disk scale length and absolute magnitude. Such a trend between galaxy luminosity (or mass) and size (as measured by either disk scale length or effective radius) has been already extensively analyzed at optical and near-IR wavelengths (Courteau \& Rix 1999; Shen et al. 2003; MacArthur et al. 2004; Barden et al. 2005; Trujillo et al. 2006; Courteau et al. 2007; Gadotti 2009; Dutton et al. 2011). It constitutes one of the basic scaling laws of galactic disks (Mo et al. 1998; Boissier \& Prantzos 2000; Firmani \& Avila-Reese 2000, 2009; Brook et al. 2006; Dutton et al. 2007, 2011; Somerville et al. 2008; Brooks et al. 2011). Observations have demonstrated that the scatter in scaling laws involving disk scale length is usually larger than in other empirical laws such as the Tully-Fisher relation (Tully \& Fisher 1977). There are several factors that may account for this.

1. In the particular case of $h$ versus circular velocity, the latter is not usually measured at $h$ but farther out, in the darkmatter-dominated regime.

2. The slope of the trend between disk scale length and luminosity seems to vary systematically along the Hubble sequence (Courteau et al. 2007).

3. The disk scale length can change with time due to secular processes, such as inside-out growth of the disk or radial stellar migration. This can blur the connection between $h$ and the dynamical properties of the halo.

\footnotetext{
25 Note that the total stellar mass includes the contribution of the bulge, should there be one.
}
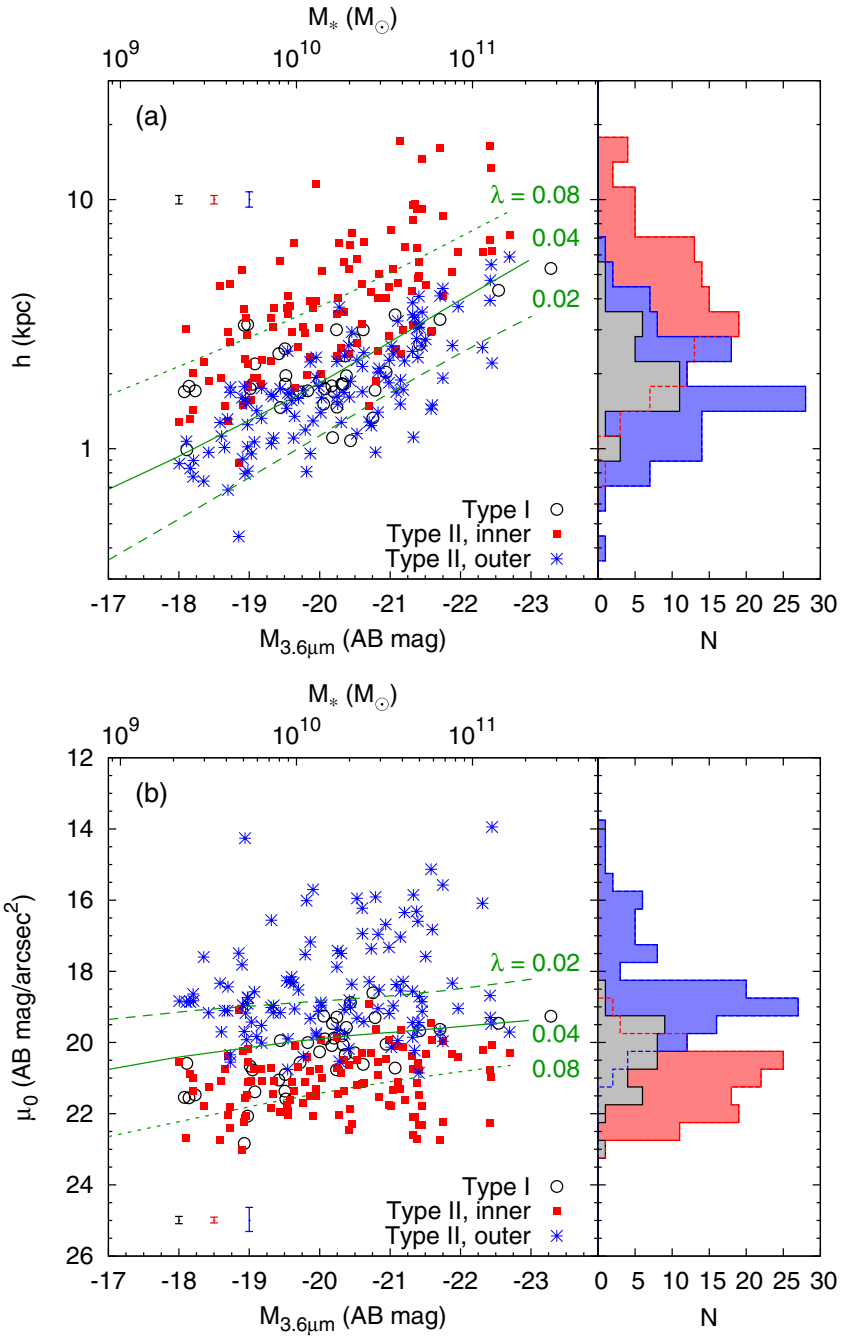

Figure 6. (a) Distribution of disk scale lengths and (b) extrapolated central surface brightness as a function of absolute magnitude at $3.6 \mu \mathrm{m}$. Singleexponential profiles are marked with open symbols. For down-bending profiles, red squares and blue diamonds correspond to the inner and outer disks, respectively. The values of $\mu_{0}$ have been corrected for inclination. Median error bars are shown with the corresponding color. The green lines show the predictions of the models of Boissier \& Prantzos (2000; mostly valid for singleexponential disks) for selected values of the spin parameter $\lambda$. The histograms to the right show the distribution of the corresponding parameters.

(A color version of this figure is available in the online journal.)

4. The scale length of a disk does not only depend on the total mass, but also on the dimensionless spin parameter $\lambda$ (see below).

5. Since most disks have more than one exponential, the notion of a single scale length is simply ill defined (see below).

Regarding issue 4, the spin parameter $\lambda$ (Peebles 1969) relates a system's angular momentum, its binding energy, and its mass, and measures the degree of rotational support. In general, for a given total mass, galaxies with higher values of $\lambda$ exhibit flatter disks (Dalcanton et al. 1997; Boissier \& Prantzos 2000). The distribution of $\lambda$ usually follows a lognormal function, both in simulations (Barnes \& Efstathiou 1987; Warren et al. 1992; Bullock et al. 2001; Gardner 2001; Vitvitska et al. 2002) and observations (Syer et al. 1999; Hernandez et al. 2007; Berta et al. 2008; Cervantes-Sodi et al. 2008; Muñoz-Mateos et al. 2011). The distribution peaks around $\lambda \sim 0.03-0.04$, with a spread that can partly account for the observed scatter in disk scale lengths for a fixed mass. 
To illustrate this, in Figure 6 we overlay the trends predicted by the models of Boissier \& Prantzos (2000). Using the $\Lambda$-CDM scaling laws as a working framework, these models incorporate analytical yet physically motivated prescriptions for radially varying gas accretion, star formation, and chemical enrichment. For any pair of values of the spin $\lambda$ and the circular velocity $V_{\mathrm{C}}$ of the parent dark matter halo, the models yield the temporal evolution of the radial profiles at different wavelengths. Figure 6 shows the predicted trends at $3.6 \mu \mathrm{m}$ at $z=0$ for different values of $\lambda$, representative of those found to fit the multiwavelength profiles of nearby disks (Muñoz-Mateos et al. 2011). By construction, the models neither implement the formation of breaks, nor do they allow for radial transfer of mass. They provide an excellent fit to the distribution of single-exponential disks in this diagram, and are thus a useful reference against which to compare down-bending profiles.

As for item 5 in the list above, up to now the connection between galaxy mass and disk scale length had been traditionally explored by assigning a single-exponential slope to each galaxy, even though most disks actually exhibit two distinct slopes. Interestingly, Figure 6(a) demonstrates that this trend still holds true when the inner and outer exponentials are considered on their own. In other words, in those galaxies with down-bending profiles, both the inner and outer disks become shallower in more massive galaxies. Moreover, even though there is some degree of overlap, single-exponential profiles define a clear boundary in this plot. The inner slope of a Type II disk will preferentially be flatter than the slope of a Type I disk with the same total mass; similarly, the outer slope will tend to be steeper. We note that this is not a trivial result, as there are many $h_{i}-h_{o}$ configurations that would yield the same total mass of a disk with a single scale length $h$.

Besides the influence of $\lambda$ and of secular processes mentioned above, this plot also explains part of the scatter in the $h-M$ trend found in previous studies. Indeed, when fitting a down-bending profile with a single-exponential function, the resulting scale length will depend on the radial position of the break and on the depth of the image. If the break happens at large radii, then most of the disk profile will be dominated by the inner disk, so that the fitted scale length will be biased toward $h_{i}$. Conversely, in those disks where the break occurs closer to the center, the light profile will be dominated by the outer exponential, thus biasing $h$ toward $h_{o}$.

\subsubsection{Extrapolated Central Surface Brightness}

Figure 6(b) shows the extrapolated central surface brightness, corrected for inclination as $\mu_{\text {corr }}=\mu_{\text {obs }}-2.5 \log (b / a)$. As happened before with the scale length, Type I disks again delineate an obvious boundary between the inner and outer parts of down-bending profiles. For a given total stellar mass, the extrapolated central surface brightness of inner (outer) disks is always fainter (brighter) than in a similarly massive Type I disk.

If we focus on single-exponential profiles alone, we can see that for galaxies brighter than $\sim-20$ (with stellar masses above $\left.10^{10} M_{\odot}\right), \mu_{0}$ remains rather constant, oscillating around $\sim 20 \mathrm{AB}$ mag $\operatorname{arcsec}^{-2}$, whereas fainter galaxies exhibit dimmer central surface brightnesses. This constancy of the central surface brightness of disks was already noted by Freeman (1970; the now called Freeman law). The scatter in that pioneering study was most likely artificially low due to selection effects, which biased observations toward high surface brightness galaxies. However, recent studies carried out on much larger samples of galaxies with deeper images have corroborated that, albeit with large scatter, $\mu_{0}$ is essentially independent of galaxy mass or Hubble type, except for the latest types. For instance, in a study of the disk structural properties of roughly $\sim 30,000$ Sloan Digital Sky Survey galaxies, Fathi (2010) found that $\mu_{0} \sim 20 \mathrm{AB}$ mag $\operatorname{arcsec}^{-2}$ in the $r$ band for early- and intermediate-type disks; this is fully consistent with our value at $3.6 \mu \mathrm{m}$, considering that $(r-3.6 \mu \mathrm{m})_{\mathrm{AB}} \sim 0.1-0.2$ for nearby spirals (Muñoz-Mateos et al. 2009). Fathi (2010) also noted that $\mu_{0}$ drops in galaxies with Hubble types $T \geqslant 6$ (Scd or later). We do observe the same trend in Figure 6, where Type I galaxies fainter than $\sim-20$ (mostly late-type ones, according to Figure 1) present indeed fainter values of $\mu_{0}$.

The Freeman law constitutes yet another constraint for disk evolution models. In particular, the models of Boissier \& Prantzos (2000) successfully reproduce the behavior of singleexponential disks (green curves in Figure 6(b)). The central surface brightness increases just mildly with the total luminosity, as observed. According to the models, most of the scatter arises from variations in $\lambda$, with high-spin disks exhibiting fainter surface brightness.

Figure 6(b) shows that the Freeman law roughly applies not only to single-exponential disks, but also to the inner component of down-bending disks. The trend is globally shifted toward fainter values by 1-2 mag, but the overall shape is the same: $\mu_{0, i}$ holds roughly constant at $\sim 21 \mathrm{AB}$ mag $\operatorname{arcsec}^{-2}$ for $M_{3.6 \mu \mathrm{m}}<-20$ and then drops for fainter disks. This behavior does not seem to be fully mirrored in the outer disks, though, perhaps because the scatter in $\mu_{0, o}$ at fixed total stellar mass is significantly larger, around 2-4 mag. Note that the extrapolated values of $\mu_{0, o}$ become increasingly more uncertain in galaxies where the break radius happens at large galactocentric distances. In these cases, a very small error in the slope of the outer disk can translate into large variations in the extrapolated central surface brightness. This can explain part of the significantly large scatter toward bright values of $\mu_{0, o}$. In general, the difference between the inner and outer central surface brightnesses is $\mu_{0, i}-\mu_{0, o} \sim 1-6 \mathrm{mag}$, in very good agreement with the simulations of Debattista et al. (2006).

\subsubsection{Disk Isophotal Radius}

Besides using disk scale lengths, another approach to look at galactic "sizes" is through the physical radius at a given surface brightness level. In Figure 7, we plot the radius at our fiducial level of $25.5 \mathrm{AB}$ mag $\operatorname{arcsec}^{-2}$ as a function of absolute $3.6 \mu \mathrm{m}$ magnitude. It can be clearly seen that both single-exponential and down-bending profiles lie along the same sequence. This means that if we plot the radial profiles of Type I and II disks with the same total stellar mass, they will roughly intersect at $\mu \sim 25.5 \mathrm{AB}$ mag $\operatorname{arcsec}^{-2}$ if the radius is in physical units.

\subsubsection{Break Radius}

Where in the disk does the change of slope occur? In Figure 8, we present the distribution of the break radius as a function of absolute magnitude. When expressed in $\mathrm{kpc}, R_{\mathrm{br}}$ is clearly correlated with galaxy luminosity, ranging from roughly $3-4 \mathrm{kpc}$ for faint galaxies with $M_{*} \sim 2 \times 10^{9} M_{\odot}$ to above $10 \mathrm{kpc}$ for those with $M_{*} \sim 10^{11} M_{\odot}$.

When normalizing the break radius by the isophotal diameter $R_{25.5}$, the trend with mass vanishes completely. The distribution of data points mildly peaks at $R_{\mathrm{br}} \sim 0.8 R_{25.5}$, although the histogram clearly exhibits extended wings, especially toward smaller break radii. The asymmetric shape of this histogram, 


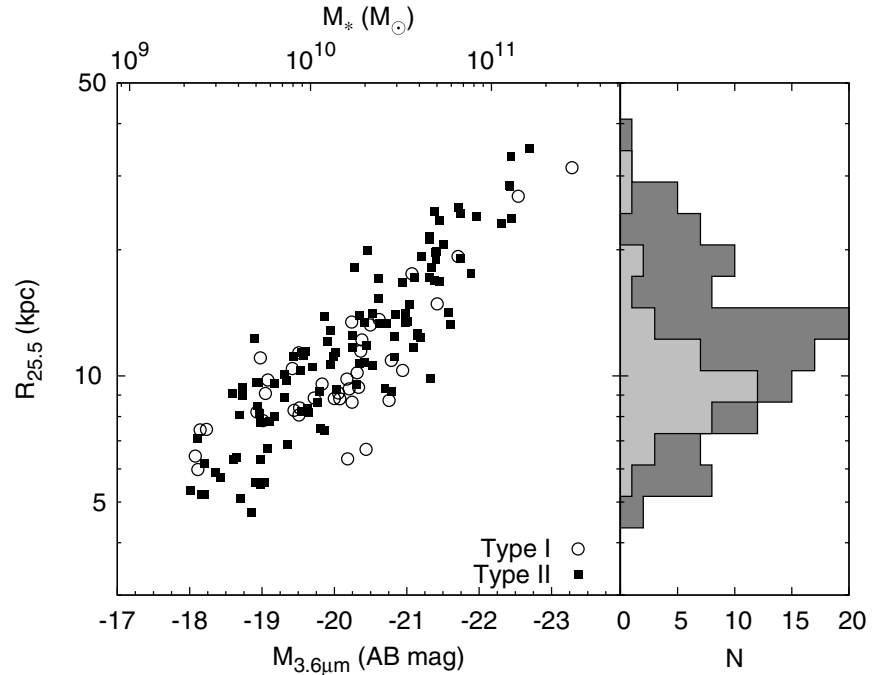

Figure 7. Radius where $\mu_{3.6 \mu \mathrm{m}}=25.5 \mathrm{AB}$ mag $\operatorname{arcsec}^{-2}$ as a function of the $3.6 \mu \mathrm{m}$ absolute magnitude, for both single-exponential disks (open circles and light histogram) and down-bending ones (black squares and dark histogram).

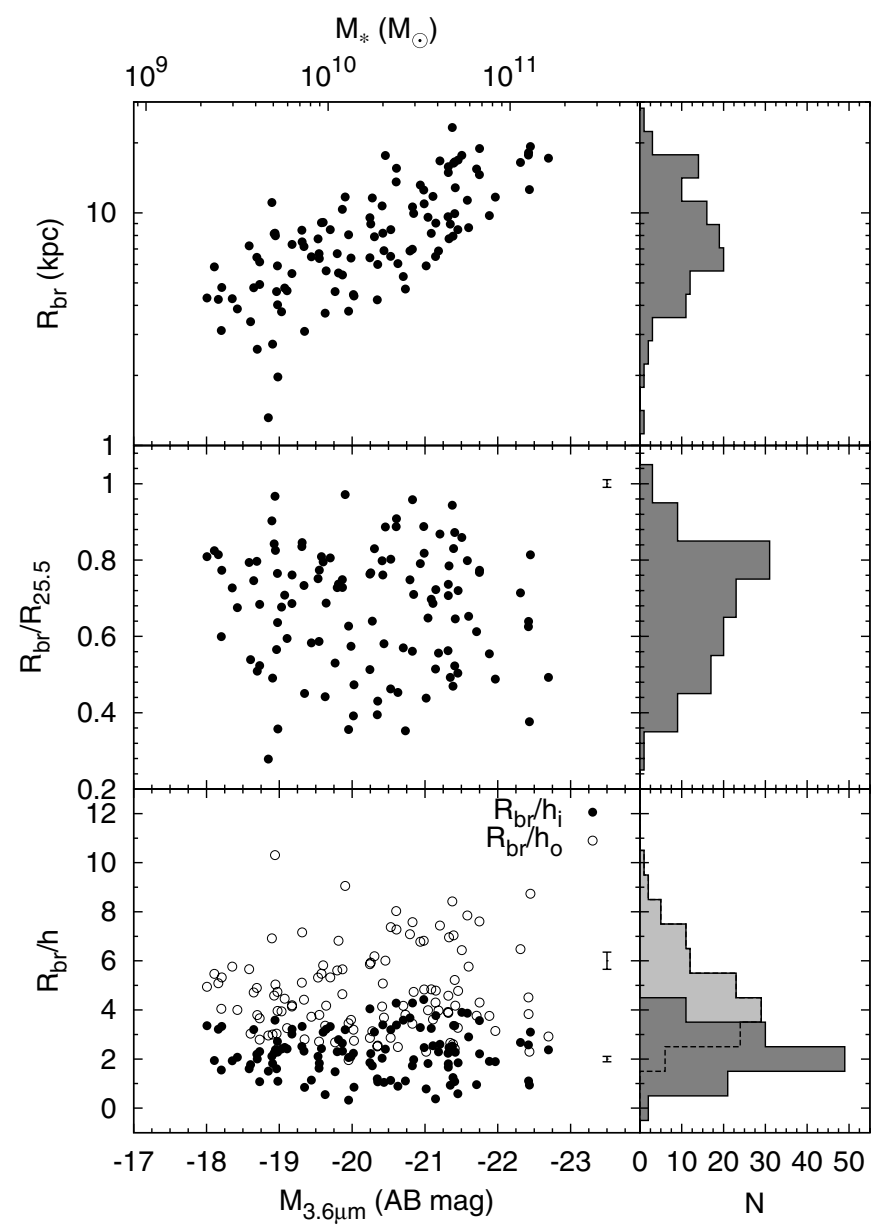

Figure 8. Distribution of the break radius as a function of the absolute $3.6 \mu \mathrm{m}$ magnitude. The break radius is shown in physical units (top panel), normalized by the radius where $\mu_{3.6 \mu \mathrm{m}}=25.5 \mathrm{AB}$ mag $\operatorname{arcsec}^{-2}$ (middle panel), and normalized by the inner $\left(h_{i}\right)$ and outer $\left(h_{o}\right)$ disk scale lengths (bottom panel). Median error bars are shown in the middle and bottom panels (they are smaller than the symbols in the top one).

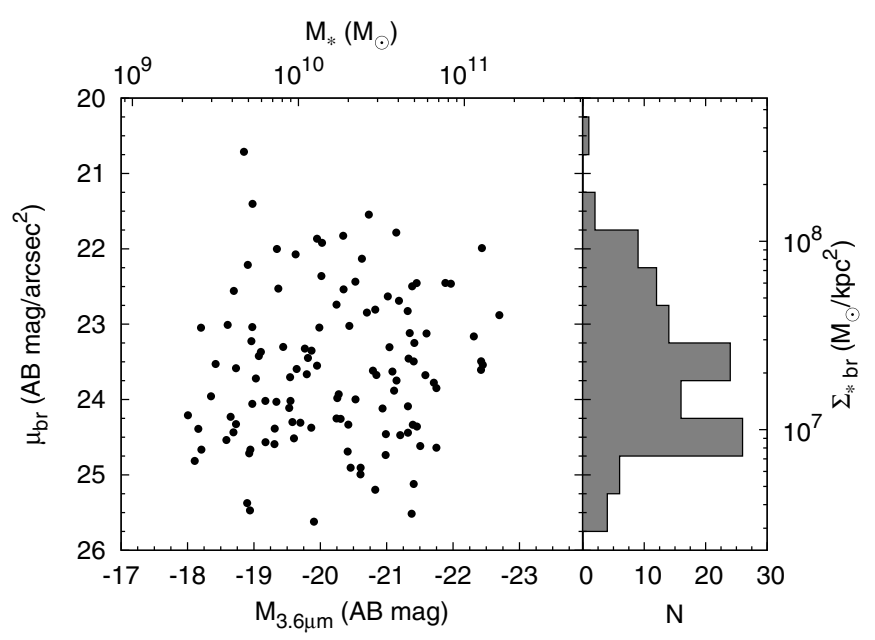

Figure 9. Deprojected 3.6 $\mu \mathrm{m}$ surface brightness at the break radius as a function of the absolute magnitude of each galaxy. The corresponding stellar mass surface density at the break and total stellar mass of the galaxy are also indicated.

which drops more abruptly above $R_{\mathrm{br}} \sim 0.8 R_{25.5}$, can be at least partly attributed to selection effects: it is obviously harder to detect breaks at large radii and low surface brightness levels, and we tend to be more conservative when identifying those breaks.

Finally, the distribution of break radii when normalized to disk scale length (both the inner and the outer one) is shown in the bottom panel of Figure 8. Again, any trend with mass seems to be washed out by the scatter. When normalizing to the inner disk scale length, the distribution appears to be strongly peaked, with a mean and rms values of $R_{\mathrm{br}}=(2.3 \pm 0.9) \times h_{i}$. The dispersion is larger in the case of the outer disk, with most breaks clustered around $R_{\mathrm{br}}=(4.7 \pm 1.7) \times h_{o}$. Our empirical distributions of $R_{\mathrm{br}} / h_{i}$ and $R_{\mathrm{br}} / h_{o}$ are consistent with those resulting from the simulations of Foyle et al. (2008).

\subsubsection{Stellar Surface Density at the Break}

Do breaks occur at a well-defined stellar mass surface density? Figure 9 shows the deprojected $3.6 \mu \mathrm{m}$ surface brightness at the position of the break. The distribution is markedly broad, with no evident dependence on the total stellar mass of the galaxy. In general, most breaks can be found anywhere in the range $\mu_{\mathrm{br}} \sim 22-25 \mathrm{AB}$ mag $\operatorname{arcsec}^{-2}$ or, equivalently, $\Sigma_{*} \sim 5 \times 10^{6}-10^{8} M_{\odot} \mathrm{kpc}^{-2}$. In their simulations of break formation and evolution, Foyle et al. (2008) found that the total baryonic (gas + stars) surface density at the break radius typically lied between $10^{7}$ and $10^{8} M_{\odot} \mathrm{kpc}^{-2}$, whereas the gas surface density alone ranged between $10^{6}$ and $10^{7} M_{\odot} \mathrm{kpc}^{-2}$. Subtracting the latter from the former yields a distribution of stellar mass surface density at the break radius entirely consistent with our findings.

\subsection{Global Properties of bars}

Now that we have broadly described the main structural properties of down-bending profiles, and compared them with equivalent single-exponential ones with the same stellar mass, we will now proceed to investigate the potential role of bars in shaping these disks. We begin with an overview of the structural properties of bars in our sample.

In Figure 10, we show the distribution of bar radii as a function of galaxy luminosity. For the sake of comparison, we plot the bar radii of both single-exponential (open circles) and down-bending profiles (solid squares). In order to distinguish 


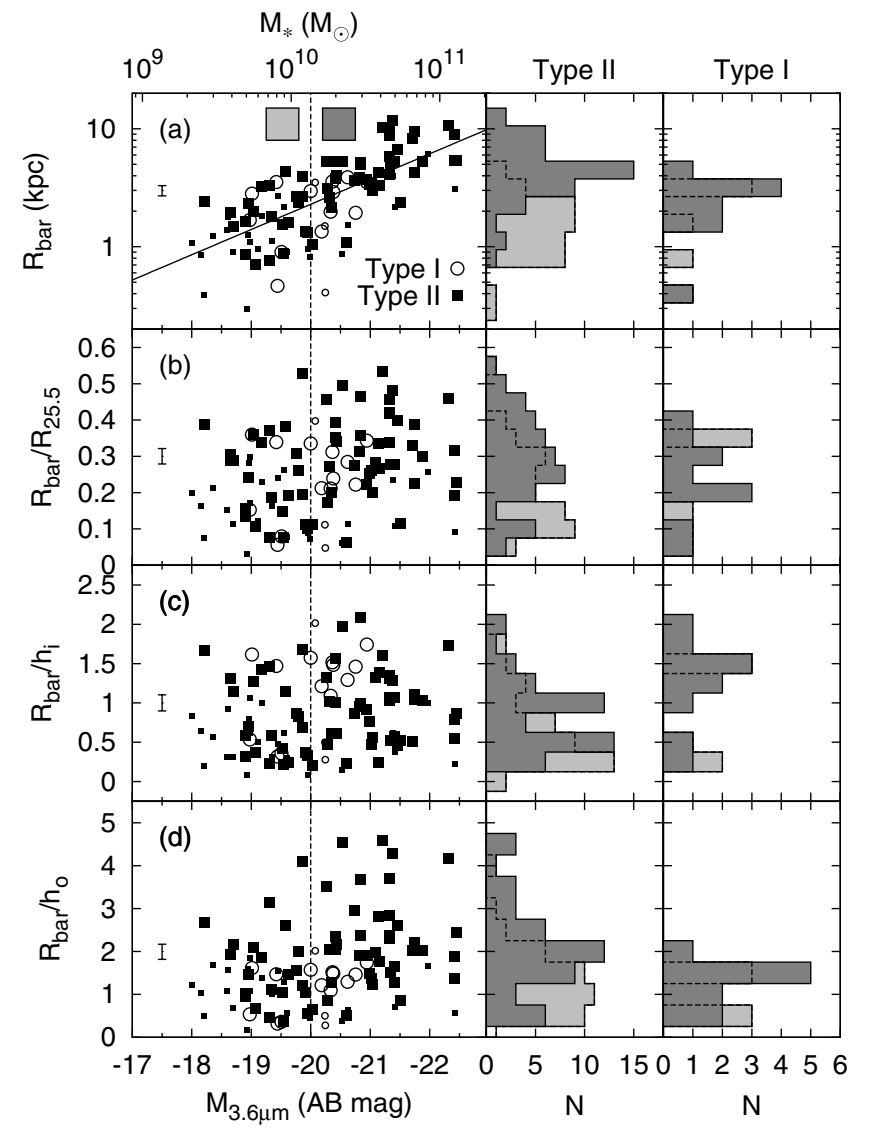

Figure 10. Bar radius as a function of absolute $3.6 \mu \mathrm{m}$ magnitude. From top to bottom, the bar radius is shown (a) in $\mathrm{kpc}$, (b) normalized by the radius where $\mu_{3.6 \mu \mathrm{m}}=25.5 \mathrm{AB}$ mag $\operatorname{arcsec}^{-2}$, (c) normalized by the scale length of the inner disk $\left(h_{i}\right)$, and (d) by that of the outer disk $\left(h_{o}\right)$. Down-bending profiles are marked with solid squares, whereas single-exponential ones are shown with open circles. Small symbols are used in either case to identify "candidate" bars. The histograms to the right show the distribution of bar radii after having divided our sample in two bins: galaxies fainter than -20 (light gray) and brighter (dark gray). Candidate bars are included in these histograms. Note that the values of $R_{\text {bar }} / h_{i}$ and $R_{\text {bar }} / h_{o}$ are exactly the same for Type I profiles, since they have a single scale length. Median error bars are shown.

"candidate" bars from "genuine" ones, we tag the former with smaller symbols. We have also divided our sample in two bins of bright and faint galaxies, taking $M_{3.6 \mu \mathrm{m}}=-20$ $\left(M_{*} \sim 10^{10} M_{\odot}\right)$ as a limiting boundary, shown in the plot with a vertical dashed line. The histograms to the right describe the distribution of bar radii for the bright and faint galaxies using a dark and light shade of gray, respectively.

Our trends between bar size and galactic mass are fully consistent with previous optical and near-IR studies of bars in the local universe (Martin 1995; Laurikainen \& Salo 2002; Erwin 2005; Menéndez-Delmestre et al. 2007). In panel (a), we can clearly see that more massive galaxies also host longer bars. This is not surprising, though, since bar length is known to correlate with disk scale length or size (e.g., Elmegreen \& Elmegreen 1985) and larger disks tend to be more massive. The linear fit in this panel corresponds to

$$
\log R_{\text {bar }}(\mathrm{kpc})=-3.920-0.214 M_{3.6 \mu \mathrm{m}}(\mathrm{AB})
$$

with a $1 \sigma$ scatter of $\sim 0.23$ dex. We will make use of this relation later in Section 4.3 when predicting the locus of different resonances.

Panel (b) shows the distribution of bar radii normalized by our reference $R_{25.5}$ radius at $3.6 \mu \mathrm{m}$. Despite the scatter,

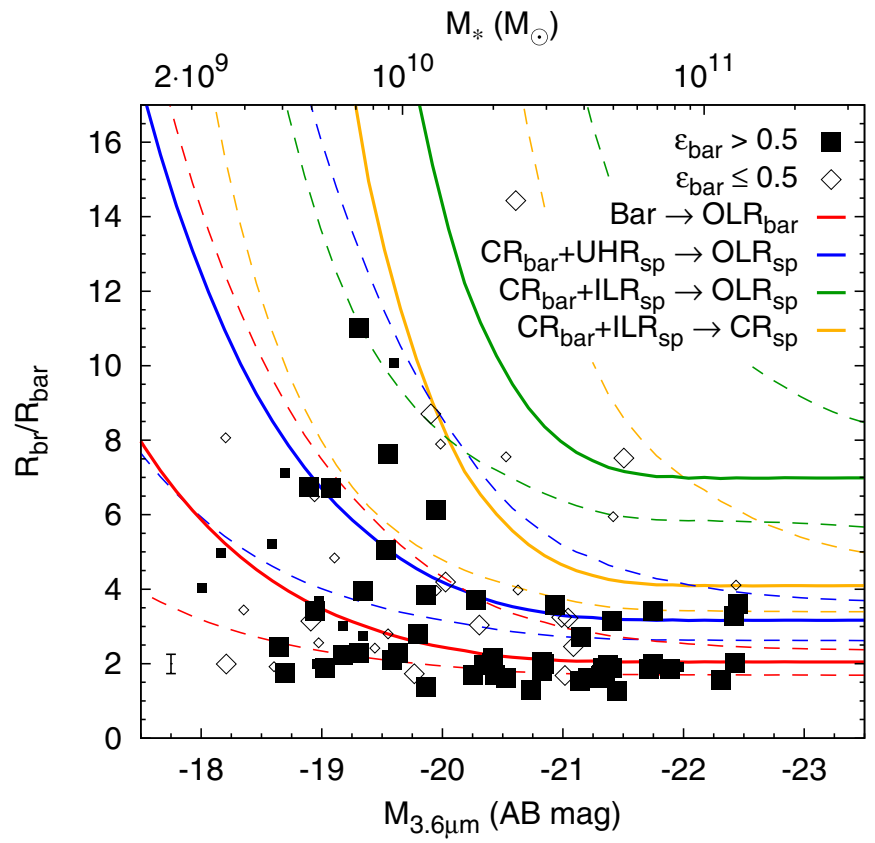

Figure 11. Break radius relative to the bar radius, as a function of absolute $3.6 \mu \mathrm{m}$ magnitude. Filled squares and open diamonds correspond to galaxies with high- and low-ellipticity bars, respectively. In both cases, small symbols denote candidate bars. The median error bar is shown in the bottom-left part. The red solid curve tracks the expected locus of $R_{\mathrm{OLR}} / R_{\mathrm{bar}}$, assuming a realistic rotation curve rather than a flat one. The blue one corresponds to the case where the inner 4:1 resonance of the spiral pattern overlaps with the bar CR, and the break happens at the spiral OLR. The green curve shows the case where the bar CR coincides with the spiral ILR, with the break being again at the spiral OLR. The orange curve has the same coupling as the green one, but assuming that the break happens at the spiral CR instead. Dashed lines account for uncertainties in the involved variables (see the text for more details).

(A color version of this figure is available in the online journal.)

a trend with mass is still visible, in the sense that bars in massive disks are longer relative to the overall disk size. The histograms demonstrate that in massive disks, bars typically have $R_{\mathrm{bar}} \sim 0.3 R_{25.5}$, while in less massive ones they tend to be half as long, with $R_{\text {bar }} \sim 0.15 R_{25.5}$ (Elmegreen \& Elmegreen 1985).

The trends are not so clear, but still present, when using disk scale length as a measuring rod against which to compare the bar size (panels (c) and (d)). Although with large scatter, bars in massive disks typically extend out to $(0.5-1) \times h_{i}$ and $2 \times h_{o}$, whereas those in low-mass disks reach out to $0.25 \times h_{i}$ and $(0.5-1) \times h_{o}$.

There is no evident difference in the distribution of bar radii between Type I and II disks. There is a hint from panels (a) and (b) that, if we restrict ourselves to faint disks (light histograms), bars would be $\sim 3$ times shorter in down-bending profiles than in single-exponential ones. This is very hard to ascertain, though, given that purely exponential profiles are so rare. Also, Type I disks lie in the upper and lower parts of panels (c) and (d), respectively, because their unique scale length $h$ is intermediate between $h_{i}$ and $h_{o}$ for a given stellar mass, as shown in Figure 6(a).

\subsection{The Connection between Bars and Breaks}

Does the bar determine the radius of the break? In Figure 11, we plot the $R_{\mathrm{br}} / R_{\mathrm{bar}}$ ratio as a function of galaxy luminosity. This diagram demonstrates that the range of possible breakto-bar ratios is strongly dependent on the total stellar mass. 


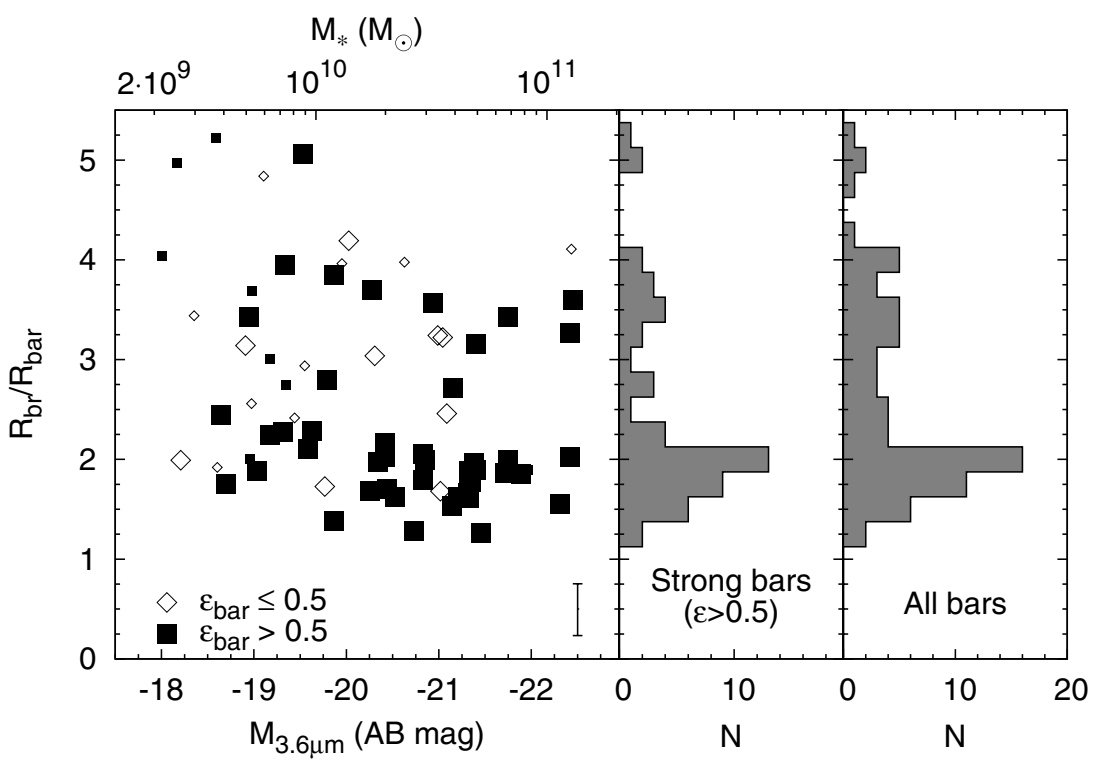

Figure 12. Enlarged plot of the lower region in Figure 11. The median error in $R_{\mathrm{br}} / R_{\mathrm{bar}}$ is shown at the bottom-right part of the plot.

Galaxies fainter than -20 (with stellar masses below $10^{10} M_{\odot}$ ) exhibit breaks at galactocentric distances ranging from 2 to more than 10 times the bar radius. More massive disks, on the other hand, are clustered around $R_{\mathrm{br}} / R_{\mathrm{bar}} \sim 2-3$; the very few ones with larger break radii tend to host bars with ellipticities lower than 0.5 , and are thus presumably weak bars.

From a purely observational perspective, this is consistent with the results of previous work (Pohlen \& Trujillo 2006; E08), where "OLR breaks" (those with $R_{\mathrm{br}} / R_{\mathrm{bar}} \sim 2-3$ ) were found to be more common in early-type disks, while "classical breaks" (at larger radii) were more abundant in latetype disks. However, we believe that this dichotomy might be too simple to fully encompass the wealth of features shown in Figure 11. Moreover, by presuming a distinct physical origin for "OLR" and "classical" breaks (resonances versus star formation (SF) thresholds), this nomenclature could be clouding our understanding of the actual physics behind these features.

Two particular issues should be noted in this regard.

1. Besides the classical OLR of the bar alone, other resonances might be involved in creating breaks. There is tantalizing evidence for this in Figure 12, which highlights the lower region of Figure 11. This plot shows that the distribution of $R_{\text {br }} / R_{\text {bar }}$ seems to be bimodal, with two separate sequences of data points clustering around either $R_{\mathrm{br}} / R_{\mathrm{bar}} \sim 2$ or $\sim 3.5$. This might be a tell-tale sign that more than one set of resonances is at play.

2 . The fact that in some cases $R_{\mathrm{br}} / R_{\mathrm{bar}} \gg 2-3$, especially in low-mass disks, does not necessarily imply that breaks arise from an SF-related mechanism rather than from a dynamical one. It is perfectly possible for a resonance to be found at large radii in these systems, as we will show.

\subsubsection{Bar-only Resonances}

To address these issues in more detail, let us consider a disk galaxy with a given rotation curve $V(r)=r \Omega(r)$. To first order, the orbit of a star can be described as the superposition of a circular orbit, with an angular velocity $\Omega(r)$, and a smaller elliptical epicycle around it, with an angular frequency $\kappa(r)$. This epicyclic frequency is given by (see, e.g., Binney \& Tremaine
2008 and references therein):

$$
\kappa^{2}=2 \Omega[2 \Omega+r(d \Omega / d r)] .
$$

When the disk hosts a non-axisymmetric pattern, such as a bar or a spiral structure, that rotates as a solid body with a given pattern speed $\Omega_{p}$, several important resonances can be found. CR occurs at the radius where stars rotate with the same angular velocity as the perturbing pattern, $\Omega=\Omega_{p}$. The Lindblad resonances are found where a star completes one epicycle between consecutive encounters with the pattern; that is, where $\Omega_{p}=\Omega \pm k / m$, with $m$ being the multiplicity of the pattern (two for a bar or a two-armed spiral). The positive sign denotes the OLR, whereas the negative one corresponds to the inner Lindblad resonance (ILR).

Under the assumption of a flat rotation curve, it is clear from the definition above that $\kappa=\sqrt{2} V / r$, and therefore $R_{\mathrm{OLR}} / R_{\mathrm{CR}}=1+1 / \sqrt{2} \simeq 1.7$. To relate this to the bar radius, we need to assume a particular value for $\mathcal{R} \equiv R_{\mathrm{CR}} / R_{\mathrm{bar}}$. Several techniques have been developed over the years to measure this ratio. These include, among others, the Tremaine-Weinberg (TW) method (Tremaine \& Weinberg 1984), matching gas flow models with observations (Sanders \& Tubbs 1980; Lindblad et al. 1996; Weiner et al. 2001) and identifying resonances with certain morphological features such as rings or dust lanes (Buta 1986; Athanassoula 1992; Elmegreen et al. 1992; Moore \& Gottesman 1995; Pérez et al. 2012). Despite building on completely different methodologies and assumptions, these techniques generally yield consistent results. They normally point toward $\mathcal{R} \simeq 1.2 \pm 0.2$, with the bar ending inside but close to corotation. This implies that $R_{\mathrm{OLR}} / R_{\mathrm{bar}} \simeq 2$, which can nicely explain the large number of breaks found at twice the bar radius, as already noted in previous works.

However, it is worth emphasizing that this estimate relies heavily on the assumption of a flat rotation curve, which is reasonable for massive disks, but might be quite far-fetched for low-mass ones, where the rotation curve rises gently for a large fraction of the optical disk. Indeed, Elmegreen \& Elmegreen (1985) showed that bars in early-type disks extend well beyond the rising part of the rotation curve, whereas bars in late-type disks end before the velocity flattens. 
The H I Nearby Galaxies Survey (Walter et al. 2008) has provided H I maps of unprecedented quality for a representative set of nearby galaxies. Rotation curves for these objects were presented by de Blok et al. (2008), and later fitted by Leroy et al. (2008) using the following analytical expression:

$$
V(r)=V_{\text {flat }}\left(1-e^{-r / r_{\text {flat }}}\right),
$$

where $V_{\text {flat }}$ is the asymptotic rotation velocity, and $r_{\text {flat }}$ is the radial scale over which the flat regime is reached. ${ }^{26}$ We fitted $r_{\text {flat }}$ as a function of the absolute magnitude at $3.6 \mu \mathrm{m}$, taken from Muñoz-Mateos et al. (2009). In general, $r_{\text {flat }}$ is around $1 \mathrm{kpc}$, and increases mildly with decreasing mass. Using this information, we can build more realistic curves of $\Omega(r)$ and $\kappa(r)$ tailored for each absolute magnitude.

From the fit in the top panel of Figure 10 (Equation (8)), we can get the typical bar radius for a galaxy of a given absolute magnitude. Multiplying this value by $\mathcal{R}=1.2$ we obtain an estimate of the CR radius, which in turn yields the corresponding OLR radius when combined with the proper rotation curve for that particular absolute magnitude, as explained above. The resulting prediction of $R_{\mathrm{OLR}} / R_{\mathrm{bar}}$ is shown as a red line in Figure 11. As expected, in the limit of high-mass galaxies we recover the value of $\sim 2$ that we derived before, since in these galaxies $R_{\text {bar }} \gg r_{\text {flat }}$. However, the OLR is found farther away from the bar in low-mass disks, where the rising nature of the rotation curve cannot be ignored given that $R_{\text {bar }} \lesssim r_{\text {flat }}$.

The dashed curves mark the estimated uncertainty in this prediction, resulting from the $1 \sigma$ scatter in the involved variables, namely,

1. scatter in $R_{\text {bar }}$ for a given $M_{3.6 \mu \mathrm{m}}: \pm 0.23$ dex (Figure 10);

2. scatter in $r_{\text {flat }}$ for a given $M_{3.6 \mu \mathrm{m}}: \pm 0.4$ dex (from the data in Leroy et al. 2008);

3. scatter in $\mathcal{R}: \pm 0.2$ (Athanassoula 1992; Elmegreen et al. 1996; Aguerri et al. 2003; Corsini 2011).

For simplicity, we have assumed that there is no degree of correlation or anticorrelation in the scatter among these variables. In practice, though, the dashed curves could be somewhat different if this assumption is not valid.

This physically motivated model shows that at least part of the scatter in $R_{\mathrm{br}} / R_{\text {bar }}$ could be simply due to the different dynamical properties of low-mass disks. Breaks in these galaxies could be perfectly well linked to the OLR of the bar; this resonance just happens to be placed further away from the bar due to the rising rotation curve of these objects.

An additional factor that we have not considered here is the fact that bars in some low-mass disks could be slow rotators, with $\mathcal{R}>1.4$ (Rautiainen et al. 2005; Meidt et al. 2009 and references therein). Large values of $\mathcal{R}$ in these objects would further increase the OLR-to-bar ratio, more than what is already depicted by the red curves.

\subsubsection{Coupled Spiral-bar Resonances}

As we mentioned before, an intriguing feature of Figures 11 and 12 is what appears to be a second family of galaxies having breaks at roughly $3.5 R_{\mathrm{bar}}$. One possible scenario worth exploring is the possibility that this reflects a coupling between the bar and spiral patterns (Tagger et al. 1987; Masset \& Tagger 1997; Sygnet et al. 1988; Rautiainen \& Salo 1999; Quillen et al. 2011; Minchev et al. 2011). Such a coupling might exist if some

\footnotetext{
26 See, e.g., Athanassoula et al. (1982) for a similar derivation of resonance
} radii with a different mathematical parameterization of the rotation curve. resonances of the bar and spiral structure overlap. In this case, the radial transfer of angular momentum can proceed farther out, leading to breaks at some point inside the OLR of the spiral rather than the OLR of the bar.

We can consider the case in which the inner 4:1 resonance of the spiral pattern (also known as the ultra harmonic resonance, UHR) overlaps with the bar corotation. Under the assumption of a flat rotation curve, one can see that the OLR of the spiral and the CR radius of the bar are such that $R_{\mathrm{OLR}, \mathrm{sp}} / R_{\mathrm{CR}, \mathrm{bar}}=$ $(1+1 / \sqrt{2}) /(1-\sqrt{2} / 4) \simeq 2.6$. Again, if we suppose that $\mathcal{R} \simeq 1.2$, then this yields $R_{\mathrm{OLR}, \mathrm{sp}} / R_{\mathrm{bar}} \simeq 3.1$, consistent with the value of $R_{\mathrm{br}} / R_{\mathrm{bar}}$ that we observe.

As we did before in the case of a bar-only OLR, we can extend this result to the generic case of a realistic rotation curve, where the radial scale $r_{\text {flat }}$ over which the curve is rising depends on the absolute magnitude of the galaxy. The resulting predicted locus for $R_{\mathrm{OLR}, \mathrm{sp}} / R_{\mathrm{bar}}$ is marked with a blue curve in Figure 11. Again, the dashed lines show the impact of the observed $1 \sigma$ variations in $R_{\text {bar }}, r_{\text {flat }}$, and $\mathcal{R}$. This figure demonstrates that, at least in principle, resonances can account for most of the observed variations in $R_{\mathrm{br}} / R_{\mathrm{bar}}$ as a function of mass, without appealing to a different mechanism for the formation of the break, such as an SF threshold. This does not necessarily mean that such thresholds do not play a role in forming breaks, but warns against systematically dismissing resonances by default whenever the break happens at more than $\sim 2 R_{\text {bar }}$.

The particular coupling between the bar and the spiral pattern described above is just one of several possible scenarios. For instance, if the bar CR overlaps with the spiral ILR, then the spiral OLR would be located even further out, as shown by the green curves in Figure 11. With this coupling, breaks would form at $\simeq 7 R_{\mathrm{bar}}$ for a flat rotation curve, and further out for a rising one. Objects in this area of the plot have low-ellipticity bars (some are even just candidate bars), so this scenario might not necessarily apply to them, but it is still a possibility worth considering.

Some simulations show that under a bar-spiral coupling the break can form at the spiral CR rather than at the OLR (Minchev et al. 2012). The orange curve illustrates this, by assuming the same coupling as the green one, but placing the break at the spiral $\mathrm{CR}$. The limiting value for a flat rotation curve ( $R_{\mathrm{br}} \simeq 4 R_{\mathrm{bar}}$ ) somewhat overpredicts the break radius for most of our massive disks, but several of our intermediate-mass disks with $M_{*} \simeq 10^{10} M_{\odot}$ and $R_{\mathrm{br}} \simeq 8 R_{\mathrm{bar}}$ could be consistent with this scenario. In brief, it is worth emphasizing that given the multiple ways in which the resonances of bars and spirals can overlap, the resulting distribution of break radii can be more complex than previously assumed.

The resonant coupling between the bar and the spiral pattern discussed above has important consequences for the redistribution of angular momentum in galactic disks. In some of their numerical simulations of disks, Debattista et al. (2006) noted that the bar and the spiral were indeed coupled via an overlap of the bar CR and the spiral UHR, like the one we propose here. In those cases, they found that the disk break took place inside but close to the OLR of the spiral, which is consistent with our results (blue curve in Figure 11). Minchev \& Famaey (2010) highlighted the impact of spiral-bar coupling in the context of radial stellar migration. They noted that the effect of such coupling in the redistribution of angular momentum is highly nonlinear, in the sense that increasing the amplitude of the bar and spiral perturbers has a significantly larger impact than 


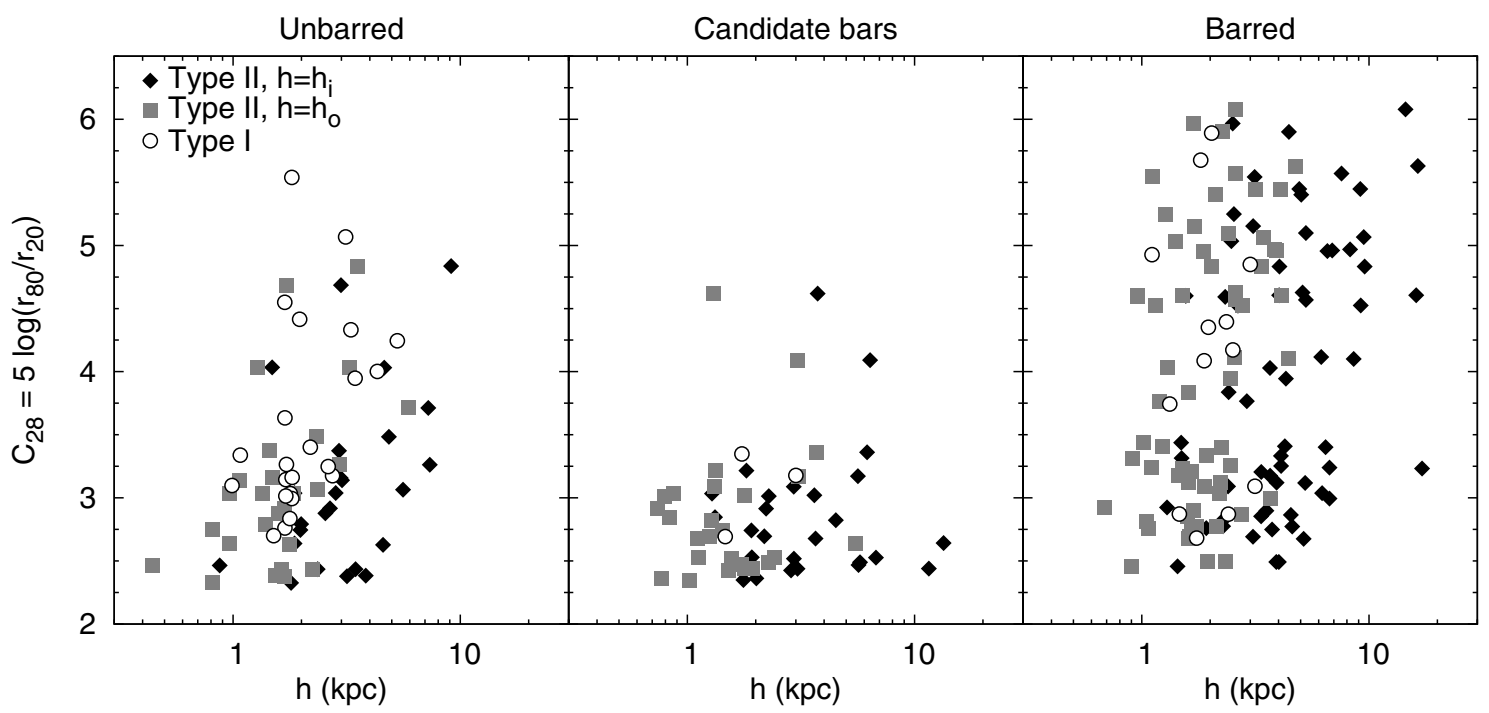

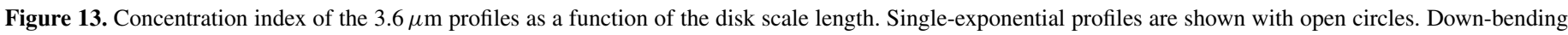

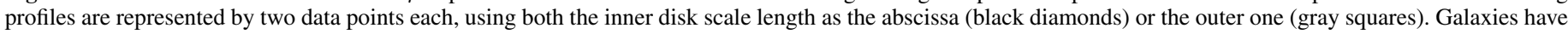
been sorted out into unbarred, candidate bars, and barred.

considering each perturber separately. As a result, this mechanism seems to be quite efficient in driving radial stellar migration. For simulations tuned to mimic the properties of a Milky Way like galaxy, Minchev \& Famaey (2010) found that after just 3 Gyr the bar-spiral coupling yields the same degree of mixing for which other mechanisms such as transient spirals would take three times longer (see below). In a follow-up study, Minchev et al. (2012) showed that this resonant coupling can in fact produce breaks at large galactocentric radii, without invoking star formation thresholds.

Studies on radial migration triggered by coupling between different patterns usually concentrate on steady-state spirals, that is, spirals that are stable over at least several rotations so that they can dynamically couple with other patterns such as the bar. Transient spiral arms constitute an alternative mechanism to drive stellar migration, as proposed by Sellwood \& Binney (2002; see also Roškar et al. 2012). Under this scenario, stars are sent from the $\mathrm{CR}$ of one spiral pattern to another, and the transient nature of the spirals prevents stars from being trapped at certain orbits. Nevertheless, these two mechanisms (transient spirals and bar-spiral coupling) are not necessarily exclusive; in fact, as discussed by Minchev \& Famaey (2010) and Minchev et al. (2012), due to its nonlinear nature, pattern coupling works with both long- and short-lived spirals.

From an observational point of view, resonant coupling between different patterns might be detected in actual galaxies using the radial TW method (Merrifield et al. 2006), a generalized modification of the TW method that allows for radial variations in the pattern speed. Meidt et al. (2009) applied this technique to a sample of nearby galaxies, and found indeed suggestive evidence for resonant coupling in some of them. Given the tantalizing signatures of potential pattern coupling seen in Figure 11, this kind of more detailed analysis is definitely worth pursuing in future papers.

Can this scenario be applied to unbarred galaxies as well? Spiral structure and spiral-spiral coupling can take place in the absence of a bar (Sygnet et al. 1988; Rautiainen \& Salo 1999), so one should also consider spiral resonances as a possible mechanism for the formation of breaks in unbarred galaxies, besides SF thresholds. Such an analysis is beyond the scope of the current paper, but will be addressed in future work.

\subsection{What Triggers the Onset of a Break?}

Foyle et al. (2008) proposed that whether a galaxy develops a break or not is largely determined by the ratio $m_{\mathrm{d}} / \lambda$, where $m_{\mathrm{d}}$ is the disk-to-halo mass fraction and $\lambda$ is the dimensionless spin parameter. In their simulations, galaxies with $m_{\mathrm{d}} / \lambda \geqslant 1$ ended up forming a break, whereas those with a low $m_{\mathrm{d}} / \lambda$ never developed one.

Confronting this hypothesis against observations is not straightforward. On one hand, computing $m_{\mathrm{d}}$ requires accurate rotation curves, which are only available for a subset of our galaxies. On the other hand, $\lambda$ cannot be directly measured from observations. While it can be indirectly inferred from the light profiles of galaxies (Muñoz-Mateos et al. 2011, and references therein), this requires neglecting radial stellar migration, which we now know can significantly reshape the light profiles of galaxies and bias the inferred values of $\lambda$.

Nevertheless, Foyle et al. (2008) noted that the concentration index of their simulated galaxies was a reasonable indicator of whether a disk would develop a break. For each galaxy they measured the temporal evolution of $C_{28}=5 \log \left(r_{80} / r_{20}\right)$, where $r_{80}$ and $r_{20}$ are the radii encompassing $20 \%$ and $80 \%$ of the total baryonic mass (Kent 1985). They found that after 5 Gyr almost all galaxies with breaks had $C_{28}>4$, whereas those with lower concentrations remained as single-exponential disks. After $10 \mathrm{Gyr}$, the concentration index separating broken and unbroken profiles had dropped to $C_{28} \sim 3.5$.

In Figure 13, we plot $C_{28}$ (as measured on the $3.6 \mu \mathrm{m}$ profiles) as a function of the disk scale length for broken profiles (filled symbols) and unbroken ones (open symbols). We also consider separately barred galaxies, unbarred ones, and candidate bars. A perfect exponential profile has $C_{28}=2.7$, but the presence of a central bulge and/or bar increases this value, as can be readily seen here. This plot should be compared with Figure 12 in Foyle et al. (2008). ${ }^{27}$ In contrast with the results of that study, here we

\footnotetext{
27 Note that these authors used the initial disk scale length of their simulated disks as their $x$-axis, which we obviously cannot do with our actual galaxies. This is not extremely relevant, though, as the parameter of interest here is $C_{28}$ along the $y$-axis. Foyle et al. (2008) measured this concentration index at 5 and 10 Gyr on the total baryonic profiles (gas + all stars). Throughout their simulations, the gas component represents a small contribution, so our stellar values of $C_{28}$ at $3.6 \mu \mathrm{m}$ constitute a good proxy.
} 
do not find that $C_{28}$ segregates broken and unbroken disks. In general, we find disks with and without breaks both above and below the theoretical limit of $C_{28} \simeq 3.5-4$ resulting from their simulations. If $C_{28}$ maps $m_{\mathrm{d}} / \lambda$ (at least to first order), then our results do not support the idea that such ratio governs the onset of disk breaks.

\section{CONCLUSIONS}

In this work, we have carried out a detailed study of the radial distribution of old stars in 218 nearby almost face-on disks, using deep $3.6 \mu \mathrm{m}$ images from $\mathrm{S}^{4} \mathrm{G}$. In particular, we have investigated the structural properties of disk breaks and their connection with bars, in order to verify whether nonaxisymmetric perturbations can lead to the observed breaks through secular rearrangement of stars and angular momentum. Our results can be summarized as follows.

1. The scale length of both the inner disk $\left(h_{i}\right)$ and the outer one $\left(h_{o}\right)$ increases monotonically with increasing total stellar mass. Interestingly, galaxies with a genuine singleexponential disk have a scale length which lies between those of the inner and outer disks of down-bending profiles with the same total stellar mass. On average we find $h_{i} / h_{o} \simeq 1-3$, which agrees with the grid of simulations by Debattista et al. (2006) and Foyle et al. (2008), among others.

2. The extrapolated central surface brightness of the inner $\left(\mu_{0, i}\right)$ and outer disks $\left(\mu_{0, o}\right)$ do not exhibit any clear trend with the total stellar mass. In this regard, they mimic the Freeman law for single-exponential disks. Again, for a given stellar mass, the central surface brightness of a single-exponential disk is intermediate between those of the inner and outer components of a down-bending disk. We typically measure $\mu_{0, i}-\mu_{0, o} \sim 1-6 \mathrm{mag}$, in agreement with the simulations of, e.g., Debattista et al. (2006).

3. The break radius ranges from $3-4 \mathrm{kpc}$ for galaxies with $M_{*} \sim 2 \times 10^{9} M_{\odot}$ to $10-20 \mathrm{kpc}$ in those with $M_{*} \sim$ $10^{11} M_{\odot}$. However, the trend vanishes when normalizing the break radius by the isophotal size of the disk or by the disk scale length. On average, most breaks occur at $R_{\mathrm{br}}=(2.3 \pm 0.9) \times h_{i}$ and $(4.7 \pm 1.7) \times h_{o}$. The stellar mass surface density at the break radius ranges between $\sim 5 \times 10^{6}-10^{8} M_{\odot} \mathrm{kpc}^{-2}$, with no trend with the mass of the host galaxy. Both the break radius and its stellar mass density agree with values found in numerical simulations (Debattista et al. 2006; Foyle et al. 2008; Roškar et al. 2008; Sánchez-Blázquez et al. 2009; Minchev et al. 2012).

4. In the particular case of barred galaxies, the ratio between the break and the bar radii presents an intriguing dependence on the total stellar mass of the galaxy. In objects less massive than $10^{10} M_{\odot}$, breaks can be found between 2 and $\sim 10$ times the bar radius. However, the scatter decreases considerably in more massive disks, where most breaks lie within 4 bar radii. This behavior is most pronounced when we consider only highly elongated bars.

5. The distribution of $R_{\mathrm{br}} / R_{\mathrm{bar}}$ in massive disks seems to be in fact bimodal. In most cases, the break takes place at twice the bar radius, which is the expected locus of the OLR of the bar, under the assumption of a flat rotation curve. However, there appears to be a second family of disks with breaks at $\sim 3.5 R_{\text {bar. }}$. We have shown that if the bar corotation radius overlaps with the inner 4:1 resonance of the spiral pattern, breaks are indeed expected to form at that radius, in agreement with numerical simulations (Debattista et al. 2006). Other combinations of resonances can produce breaks at even larger radii. Such resonant coupling between different patterns is most relevant in the context of secular evolution of disks, since numerical simulations have demonstrated that radial stellar migration becomes more efficient in this case (Minchev \& Famaey 2010; Minchev et al. 2011, 2012).

6. It is normally assumed that resonances cannot be responsible for those breaks found at $R_{\mathrm{br}} \gg 2 \times R_{\mathrm{bar}}$, and other mechanisms such as SF thresholds are usually invoked. However, this kind of breaks occur mostly in low-mass disks, where bars end before the rotation curve has reached the flat regime. This tends to push the aforementioned resonances further out, compared to the case of a flat rotation curve, which is more suitable for massive disks. Using average but realistic rotation curves as a function of the total stellar mass, we have shown that resonances with the bar and/or the spiral pattern can account for the increased scatter in $R_{\mathrm{br}} / R_{\mathrm{bar}}$ at low masses. While this does not rule out SF thresholds as a break formation mechanism, it cautions against discarding resonances by default whenever a break happens far away from the bar.

7. It has been proposed that the development of breaks is governed by $m_{\mathrm{d}} / \lambda$, the ratio between the disk mass fraction and the halo spin parameter (Foyle et al. 2008). While such quantity cannot be easily measured, the light concentration index $C_{28}$ has been brought forward as a reasonable proxy. We find, however, no connection between high/low values of $C_{28}$ and the presence/absence of a break.

The authors are grateful to the entire $S^{4} G$ team for their collective effort in this project. We thank S. Boissier for allowing us to use his disk evolution models. We also thank the referee for a very constructive report that helped to improve the quality of the paper. J.C.M.M. acknowledges financial support from NASA JPL/Spitzer Grant RSA 1374189 provided for the $S^{4} G$ project. J.C.M.M., K.S., and T.K. acknowledge support from the National Radio Astronomy Observatory, which is a facility of the National Science Foundation operated under cooperative agreement by Associated Universities, Inc. We acknowledge financial support from the People Programme (Marie Curie Actions) of the European Union's FP7/2007-2013/ to the DAGAL network under REA grant agreement No. PITN-GA2011-289313.

This work is based on data acquired with the Spitzer Space Telescope, and makes extensive use of the NASA/IPAC Extragalactic Database (NED), both of which are operated by the Jet Propulsion Laboratory, California Institute of Technology under a contract with the National Aeronautics and Space Administration (NASA). We also acknowledge the usage of the HyperLeda database (http://leda.univ-lyon1.fr).

Facilities: Spitzer

\section{APPENDIX A}

\section{MASS-TO-LIGHT RATIO AT $3.6 \mu \mathrm{m}$}

Throughout this paper we have relied on the mass-tolight ratio at $3.6 \mu \mathrm{m}$ of Eskew et al. (2012). These authors compared spatially resolved stellar mass maps of the Large 

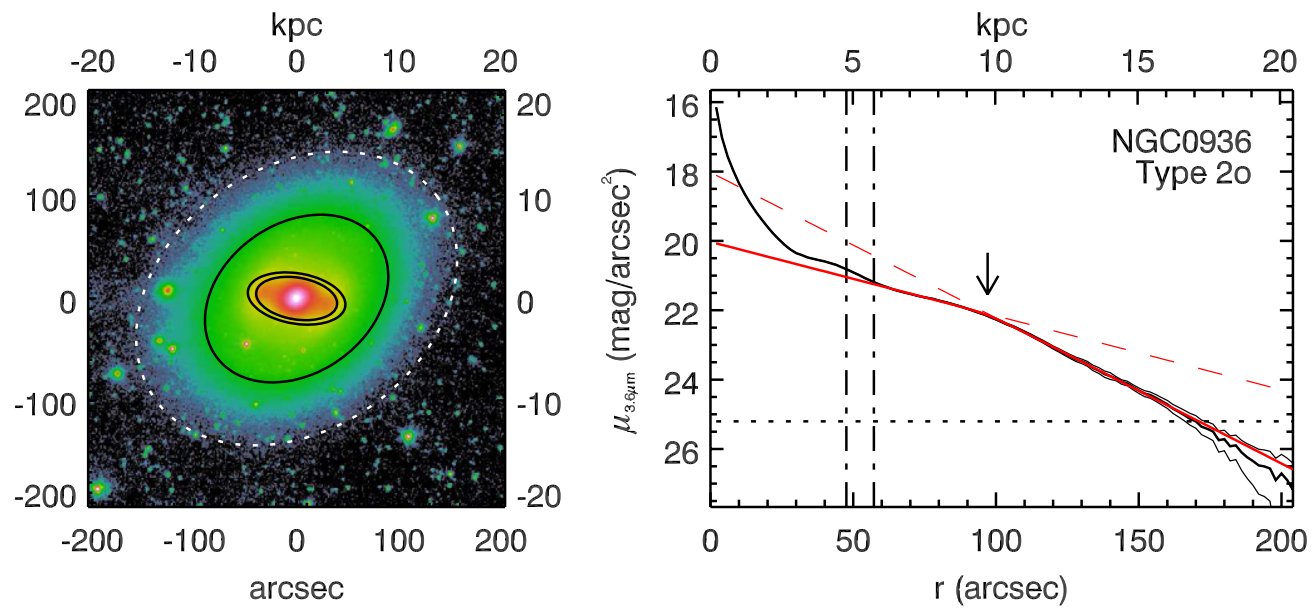

Figure 14. Image and surface brightness profile for NGC 0936 at $3.6 \mu \mathrm{m}$. The best-fitting disk model (Section 3.3) is shown as a red solid curve; for clarity, red dashed lines indicate the extrapolation of the inner and outer components of the profile. The position of the break is marked with a vertical arrow in the profile and a black solid ellipse in the image. The limits for the bar radius (Section 3.4) are shown as two vertical dash-dotted lines and two concentric ellipses. The ellipticity and P.A. of these two ellipses are those at $r=a_{\epsilon \max }$. In the case of "candidate bars," the vertical lines have a dash-dot-dot-dotted pattern. The horizontal dotted line and the white dotted ellipse mark the radius beyond which $\Delta \mu>0.2 \mathrm{mag} \mathrm{arcsec}^{-2}$.

(A color version and the complete figure set (227 images) of this figure are available in the online journal.)

Magellanic Cloud with the corresponding IRAC maps to derive the following calibration:

$$
\frac{M_{*}}{M_{\odot}}=10^{5.97} \frac{F_{3.6}}{\mathrm{Jy}}\left(\frac{D / \mathrm{Mpc}}{0.05}\right)^{2}
$$

or, equivalently,

$$
\log M_{*} / M_{\odot}=-0.4 M_{3.6 \mathrm{AB}}+2.13
$$

They also provide the following calibration when fluxes at both $3.6 \mu \mathrm{m}$ and $4.5 \mu \mathrm{m}$ are available:

$$
\frac{M_{*}}{M_{\odot}}=10^{5.65} \frac{F_{3.6}^{2.85}}{\mathrm{Jy}} \frac{F_{4.5}^{-1.85}}{\mathrm{Jy}}\left(\frac{D / \mathrm{Mpc}}{0.05}\right)^{2} .
$$

Galaxies in our sample exhibit a very narrow range of 3.6-4.5 colors, the average value being $-0.41 \pm 0.08 \mathrm{mag}(\mathrm{AB})$. Substituting this value into Equation (A3) yields

$$
\log M_{*} / M_{\odot}=-0.4 M_{3.6 \mathrm{AB}}+2.12 \pm 0.06
$$

which is entirely consistent with Equation (A2). The corresponding stellar mass surface density is given by

$$
\log \Sigma_{*}\left(M_{\odot} \mathrm{kpc}^{-2}\right)=16.76-0.4 \mu_{3.6}\left(\mathrm{AB} \operatorname{mag} \operatorname{arcsec}^{-2}\right) .
$$

\section{APPENDIX B}

\section{RADIAL PROFILES}

To facilitate the visual inspection of the structural properties of our galaxies, in this appendix we compile the images and radial profiles for the whole sample, overplotting the position of the breaks and bars when present. The whole set of figures can be found in the online version of the journal (Figure 14).

\section{REFERENCES}

Aguerri, J. A. L., Debattista, V. P., \& Corsini, E. M. 2003, MNRAS, 338, 465 Athanassoula, E. 1992, MNRAS, 259, 345

Athanassoula, E. 2002, ApJL, 569, L83

Athanassoula, E. 2003, MNRAS, 341, 1179
Athanassoula, E. 2012, MNRAS, 426, L46

Athanassoula, E., Bosma, A., Creze, M., \& Schwarz, M. P. 1982, A\&A, 107,101

Athanassoula, E., \& Misiriotis, A. 2002, MNRAS, 330, 35

Athanassoula, E., \& Sellwood, J. A. 1986, MNRAS, 221, 213

Azzollini, R., Trujillo, I., \& Beckman, J. E. 2008a, ApJL, 679, L69

Azzollini, R., Trujillo, I., \& Beckman, J. E. 2008b, ApJ, 684, 1026

Bakos, J., Trujillo, I., \& Pohlen, M. 2008, ApJL, 683, L103

Barden, M., Rix, H.-W., Somerville, R. S., et al. 2005, ApJ, 635, 959

Barnes, J., \& Efstathiou, G. 1987, ApJ, 319, 575

Berta, Z. K., Jimenez, R., Heavens, A. F., \& Panter, B. 2008, MNRAS, 391, 197

Bertin, E., \& Arnouts, S. 1996, A\&AS, 117, 393

Binney, J., \& Tremaine, S. 2008, Galactic Dynamics (2nd ed.; Princeton, NJ: Princeton Univ. Press),

Boissier, S., \& Prantzos, N. 2000, MNRAS, 312, 398

Boselli, A., Tuffs, R. J., Gavazzi, G., Hippelein, H., \& Pierini, D. 1997, A\&AS, 121, 507

Brinchmann, J., Charlot, S., White, S. D. M., et al. 2004, MNRAS, 351, 1151

Brook, C. B., Kawata, D., Martel, H., Gibson, B. K., \& Bailin, J. 2006, ApJ, 639,126

Brooks, A. M., Solomon, A. R., Governato, F., et al. 2011, ApJ, 728, 51

Bullock, J. S., Dekel, A., Kolatt, T. S., et al. 2001, ApJ, 555, 240

Buta, R. 1986, ApJS, 61, 609

Buta, R., \& Block, D. L. 2001, ApJ, 550, 243

Buta, R., \& Crocker, D. A. 1993, AJ, 105, 1344

Buta, R., Laurikainen, E., Salo, H., Block, D. L., \& Knapen, J. H. 2006, AJ, 132,1859

Cervantes-Sodi, B., Hernandez, X., Park, C., \& Kim, J. 2008, MNRAS, 388,863

Christlein, D., \& Zaritsky, D. 2008, ApJ, 680, 1053

Combes, F., \& Sanders, R. H. 1981, A\&A, 96, 164

Comerón, S., Elmegreen, B. G., Knapen, J. H., et al. 2011a, ApJ, 741, 28

Comerón, S., Elmegreen, B. G., Salo, H., et al. 2012, ApJ, 759, 98

Comerón, S., Knapen, J. H., Beckman, J. E., et al. 2010, MNRAS, 402, 2462

Comerón, S., Knapen, J. H., Sheth, K., et al. 2011b, ApJ, 729, 18

Corsini, E. M. 2011, MSAIS, 18, 23

Courteau, S., Dutton, A. A., van den Bosch, F. C., et al. 2007, ApJ, 671, 203

Courteau, S., \& Rix, H.-W. 1999, ApJ, 513, 561

Dalcanton, J. J., Spergel, D. N., \& Summers, F. J. 1997, ApJ, 482, 659

Debattista, V. P., Mayer, L., Carollo, C. M., et al. 2006, ApJ, 645, 209

de Blok, W. J. G., Walter, F., Brinks, E., et al. 2008, AJ, 136, 2648

de Jong, R. S., Seth, A. C., Radburn-Smith, D. J., et al. 2007, ApJL, 667, L49

de Souza, R. E., Gadotti, D. A., \& dos Anjos, S. 2004, ApJS, 153, 411

Dutton, A. A., van den Bosch, F. C., Dekel, A., \& Courteau, S. 2007, ApJ, 654, 27

Dutton, A. A., van den Bosch, F. C., Faber, S. M., et al. 2011, MNRAS, 410,1660

Elmegreen, B. G., \& Elmegreen, D. M. 1985, ApJ, 288, 438 
Elmegreen, B. G., Elmegreen, D. M., Chromey, F. R., Hasselbacher, D. A., \& Bissell, B. A. 1996, AJ, 111, 2233

Elmegreen, B. G., Elmegreen, D. M., \& Montenegro, L. 1992, ApJS, 79, 37

Elmegreen, B. G., \& Hunter, D. A. 2006, ApJ, 636, 712

Erwin, P. 2005, MNRAS, 364, 283

Erwin, P., Beckman, J. E., \& Pohlen, M. 2005, ApJL, 626, L81

Erwin, P., Pohlen, M., \& Beckman, J. E. 2008, AJ, 135, 20

Eskew, M., Zaritsky, D., \& Meidt, S. 2012, AJ, 143, 139

Eskridge, P. B., Frogel, J. A., Pogge, R. W., et al. 2000, AJ, 119, 536

Fathi, K. 2010, ApJL, 722, L120

Firmani, C., \& Avila-Reese, V. 2000, MNRAS, 315, 457

Firmani, C., \& Avila-Reese, V. 2009, MNRAS, 396, 1675

Foyle, K., Courteau, S., \& Thacker, R. J. 2008, MNRAS, 386, 1821

Freeman, K. C. 1970, ApJ, 160, 811

Gadotti, D. A. 2008, MNRAS, 384, 420

Gadotti, D. A. 2009, MNRAS, 393, 1531

Gadotti, D. A., Athanassoula, E., Carrasco, L., et al. 2007, MNRAS, 381, 943

Gardner, J. P. 2001, ApJ, 557, 616

Gil de Paz, A., \& Madore, B. F. 2005, ApJS, 156, 345

Gil de Paz, A., Madore, B. F., Boissier, S., et al. 2005, ApJL, 627, L29

Hernandez, X., Park, C., Cervantes-Sodi, B., \& Choi, Y.-Y. 2007, MNRAS, 375,163

Hoyle, B., Masters, K. L., Nichol, R. C., et al. 2011, MNRAS, 415, 3627

Hubble, E. P. 1926, ApJ, 64, 321

Hunter, D. A., \& Elmegreen, B. G. 2006, ApJS, 162, 49

Kauffmann, G., Heckman, T. M., White, S. D. M., et al. 2003, MNRAS, 341,54

Kennicutt, R. C., Jr. 1989, ApJ, 344, 685

Kent, S. M. 1985, ApJS, 59, 115

Knapen, J. H., Shlosman, I., \& Peletier, R. F. 2000, ApJ, 529, 93

Kormendy, J. 1979, ApJ, 227, 714

Laine, S., Shlosman, I., Knapen, J. H., \& Peletier, R. F. 2002, ApJ, 567, 97

Laurikainen, E., \& Salo, H. 2002, MNRAS, 337, 1118

Laurikainen, E., Salo, H., \& Buta, R. 2005, MNRAS, 362, 1319

Laurikainen, E., Salo, H., Buta, R., \& Knapen, J. H. 2007, MNRAS, 381, 401

Laurikainen, E., Salo, H., \& Rautiainen, P. 2002, MNRAS, 331, 880

Lemonias, J. J., Schiminovich, D., Thilker, D., et al. 2011, ApJ, 733, 74

Leroy, A. K., Walter, F., Brinks, E., et al. 2008, AJ, 136, 2782

Lindblad, P. A. B., Lindblad, P. O., \& Athanassoula, E. 1996, A\&A, 313, 65

MacArthur, L. A., Courteau, S., Bell, E., \& Holtzman, J. A. 2004, ApJS, 152,175

Marinova, I., \& Jogee, S. 2007, ApJ, 659, 1176

Martin, P. 1995, AJ, 109, 2428

Martín-Navarro, I., Bakos, J., Trujillo, I., et al. 2012, MNRAS, 427, 1102

Masset, F., \& Tagger, M. 1997, A\&A, 322, 442

Masters, K. L., Nichol, R. C., Hoyle, B., et al. 2011, MNRAS, 411, 2026

Meidt, S. E., Rand, R. J., \& Merrifield, M. R. 2009, ApJ, 702, 277

Meidt, S. E., Schinnerer, E., Knapen, J. H., et al. 2012, ApJ, 744, 17

Menéndez-Delmestre, K., Sheth, K., Schinnerer, E., Jarrett, T. H., \& Scoville,

N. Z. 2007, ApJ, 657, 790

Merrifield, M. R., Rand, R. J., \& Meidt, S. E. 2006, MNRAS, 366, L17

Minchev, I., \& Famaey, B. 2010, ApJ, 722, 112

Minchev, I., Famaey, B., Combes, F., et al. 2011, A\&A, 527, A147

Minchev, I., Famaey, B., Quillen, A. C., et al. 2012, A\&A, 548, A126

Mo, H. J., Mao, S., \& White, S. D. M. 1998, MNRAS, 295, 319

Moore, E. M., \& Gottesman, S. T. 1995, ApJ, 447, 159

Muñoz-Mateos, J. C., Boissier, S., Gil de Paz, A., et al. 2011, ApJ, 731, 10

Muñoz-Mateos, J. C., Gil de Paz, A., Zamorano, J., et al. 2009, ApJ, 703, 1569

Nair, P. B., \& Abraham, R. G. 2010, ApJL, 714, L260

Ohta, K., Hamabe, M., \& Wakamatsu, K.-I. 1990, ApJ, 357, 71

Oke, J. B. 1974, ApJS, 27, 21
Paturel, G., Petit, C., Prugniel, P., et al. 2003, A\&A, 412, 45

Peebles, P. J. E. 1969, ApJ, 155, 393

Peng, C. Y., Ho, L. C., Impey, C. D., \& Rix, H.-W. 2002, AJ, 124, 266

Pérez, I. 2004, A\&A, 427, L17

Pérez, I., Aguerri, J. A. L., \& Méndez-Abreu, J. 2012, A\&A, 540, A103

Pohlen, M., Dettmar, R.-J., Lütticke, R., \& Aronica, G. 2002, A\&A, 392, 807

Pohlen, M., \& Trujillo, I. 2006, A\&A, 454, 759

Prieto, M., Aguerri, J. A. L., Varela, A. M., \& Muñoz-Tuñón, C. 2001, A\&A, 367,405

Quillen, A. C., Dougherty, J., Bagley, M. B., Minchev, I., \& Comparetta, J. 2011, MNRAS, 417, 762

Quillen, A. C., Frogel, J. A., \& Gonzalez, R. A. 1994, ApJ, 437, 162

Radburn-Smith, D. J., de Jong, R. S., Seth, A. C., et al. 2011, ApJS, 195, 18

Rautiainen, P., \& Salo, H. 1999, A\&A, 348, 737

Rautiainen, P., Salo, H., \& Laurikainen, E. 2005, ApJL, 631, L129

Reese, A. S., Williams, T. B., Sellwood, J. A., Barnes, E. I., \& Powell, B. A. 2007, AJ, 133, 2846

Roškar, R., Debattista, V. P., Quinn, T. R., \& Wadsley, J. 2012, MNRAS, 426, 2089

Roškar, R., Debattista, V. P., Stinson, G. S., et al. 2008, ApJL, 675, L65

Sánchez-Blázquez, P., Courty, S., Gibson, B. K., \& Brook, C. B. 2009, MNRAS, 398, 591

Sanders, R. H., \& Tubbs, A. D. 1980, ApJ, 235, 803

Schiminovich, D., Wyder, T. K., Martin, D. C., et al. 2007, ApJS, 173, 315

Sellwood, J. A. 1980, A\&A, 89, 296

Sellwood, J. A., \& Binney, J. J. 2002, MNRAS, 336, 785

Shen, S., Mo, H. J., White, S. D. M., et al. 2003, MNRAS, 343, 978

Sheth, K., Elmegreen, D. M., Elmegreen, B. G., et al. 2008, ApJ, 675, 1141

Sheth, K., Melbourne, J., Elmegreen, D. M., et al. 2012, ApJ, 758, 136

Sheth, K., Regan, M., Hinz, J. L., et al. 2010, PASP, 122, 1397

Sheth, K., Vogel, S. N., Regan, M. W., et al. 2002, AJ, 124, 2581

Skibba, R. A., Masters, K. L., Nichol, R. C., et al. 2012, MNRAS, 423, 1485

Somerville, R. S., Barden, M., Rix, H.-W., et al. 2008, ApJ, 672, 776

Syer, D., Mao, S., \& Mo, H. J. 1999, MNRAS, 305, 357

Sygnet, J. F., Tagger, M., Athanassoula, E., \& Pellat, R. 1988, MNRAS, 232,733

Tagger, M., Sygnet, J. F., Athanassoula, E., \& Pellat, R. 1987, ApJL, 318, L43

Thilker, D. A., Bianchi, L., Boissier, S., et al. 2005, ApJL, 619, L79

Thilker, D. A., Boissier, S., Bianchi, L., et al. 2007, ApJS, 173, 572

Tremaine, S., \& Weinberg, M. D. 1984, ApJL, 282, L5

Tremonti, C. A., Heckman, T. M., Kauffmann, G., et al. 2004, ApJ, 613, 898

Trujillo, I., Förster Schreiber, N. M., Rudnick, G., et al. 2006, ApJ, 650, 18

Trujillo, I., \& Pohlen, M. 2005, ApJL, 630, L17

Tully, R. B., \& Fisher, J. R. 1977, A\&A, 54, 661

van der Kruit, P. C. 1979, A\&AS, 38, 15

van der Kruit, P. C. 1987, A\&A, 173, 59

Vitvitska, M., Klypin, A. A., Kravtsov, A. V., et al. 2002, ApJ, 581, 799

Walter, F., Brinks, E., de Blok, W. J. G., et al. 2008, AJ, 136, 2563

Warren, M. S., Quinn, P. J., Salmon, J. K., \& Zurek, W. H. 1992, ApJ, 399,405

Weiner, B. J., Sellwood, J. A., \& Williams, T. B. 2001, ApJ, 546, 931

Whyte, L. F., Abraham, R. G., Merrifield, M. R., et al. 2002, MNRAS, 336, 1281

Wozniak, H., Friedli, D., Martinet, L., Martin, P., \& Bratschi, P. 1995, A\&AS, 111,115

Yoachim, P., Roškar, R., \& Debattista, V. P. 2010, ApJL, 716, L4

Yoachim, P., Roškar, R., \& Debattista, V. P. 2012, ApJ, 752, 97

Younger, J. D., Cox, T. J., Seth, A. C., \& Hernquist, L. 2007, ApJ, 670, 269

Zaritsky, D., \& Christlein, D. 2007, AJ, 134, 135

Zaritsky, D., \& Lo, K. Y. 1986, ApJ, 303, 66

Zibetti, S., \& Groves, B. 2011, MNRAS, 417, 812 
ERRATUM: “THE IMPACT OF BARS ON DISK BREAKS AS PROBED BY Sª IMAGING” (2013, ApJ, 771, 59)

Juan Carlos Muñoz-Mateos ${ }^{1}$, Kartik Sheth ${ }^{1}$, Armando Gil de Paz ${ }^{2}$, Sharon Meidt ${ }^{3}$, E. Athanassoula ${ }^{4}$, Albert Bosma $^{4}$, Sébastien Comerón ${ }^{5}$, Debra M. Elmegreen ${ }^{6}$, Bruce G. Elmegreen $^{7}$, Santiago Erroz-Ferrer ${ }^{8,9}$ Dimitri A. Gadotti ${ }^{10}$, Joannah L. Hinz ${ }^{11}$, Luis C. Ho ${ }^{12}$, Benne Holwerda ${ }^{13}$, Thomas H. Jarrett ${ }^{14}$, Taehyun Kim ${ }^{1,10,12,15}$, Johan H. Knapen ${ }^{8,9}$, JarkKo Laine ${ }^{5}$, Eija Laurikainen ${ }^{5,16}$, Barry F. Madore ${ }^{12}$, Karin Menendez-Delmestre ${ }^{17}$, Trisha Mizusawa ${ }^{1,18}$, Michael Regan ${ }^{19}$, Heikki Salo ${ }^{5}$, Eva Schinnerer ${ }^{3}$, Michael Seibert ${ }^{12}$, Ramin Skibba ${ }^{11}$, and DENNIS ZARITSKY ${ }^{11}$

${ }^{1}$ National Radio Astronomy Observatory/NAASC, 520 Edgemont Road, Charlottesville, VA 22903; jmunoz@nrao.edu

${ }^{2}$ Departamento de Astrofísica, Universidad Complutense de Madrid, Madrid 28040, Spain

${ }^{3}$ Max-Planck-Institut für Astronomie, Königstuhl 17, 69117 Heidelberg, Germany

${ }^{4}$ Aix Marseille Universite, CNRS, LAM (Laboratoire d'Astrophysique de Marseille) UMR 7326, 13388, Marseille, France

${ }^{5}$ Department of Physical Sciences/Astronomy Division, University of Oulu, FIN-90014, Finland

${ }^{6}$ Vassar College, Dept. of Physics and Astronomy, Poughkeepsie, NY 12604

${ }^{7}$ IBM Research Division, T.J. Watson Research Center, Yorktown Hts., NY 10598

${ }^{8}$ Instituto de Astrofísica de Canarias, E-38205 La Laguna, Spain

${ }^{9}$ Departamento de Astrofísica, Universidad de La Laguna, 38206 La Laguna, Spain

${ }^{10}$ European Southern Observatory, Casilla 19001, Santiago 19, Chile

${ }^{11}$ University of Arizona, 933 N. Cherry Ave, Tucson, AZ 85721

12 The Observatories, Carnegie Institution of Washington, 813 Santa Barbara Street, Pasadena, CA 91101

${ }_{13}$ European Space Agency, ESTEC, Keplerlaan 1, 2200 AG, Noordwijk, the Netherlands

${ }^{14}$ Astrophysics, Cosmology and Gravity Centre, Department of Astronomy, University of Cape Town, Private Bag X3, Rondebosch 7701, South Africa

${ }_{15}$ Astronomy Program, Department of Physics and Astronomy, Seoul National University, Seoul 151-742, Korea

${ }^{17}$ Observatorio do Valongo, Universidade Federal de Rio de Janeiro, Ladeira Pedro Antonio, 43, Saude CEP 20080-090, Rio de Janeiro-RJ-Brasil

${ }^{18}$ Florida Institute of Technology, Melbourne, FL 32901

${ }^{19}$ Space Telescope Science Institute, 3700 San Martin Drive, Baltimore, MD 21218

Received 2016 January 15; accepted 2016 January 20; published 2016 February 10

In Equation (3), the zero-point to convert the surface brightness from $\mathrm{MJy} \mathrm{str}^{-1}$ to $\mathrm{AB} \mathrm{mag} \operatorname{arcsec}^{-2}$ is incorrect. The correct value is 20.472:

$$
\mu_{\text {corr }}\left(\mathrm{AB} \text { mag } \operatorname{arcsec}{ }^{-2}\right)=-2.5 \log \left[I_{\text {corr }}\left(\mathrm{MJy} \mathrm{str}^{-1}\right)\right]+20.472
$$

This is just a typo that does not affect the radial profiles nor the integrated magnitudes presented here, which were correctly computed. The complete $\mathrm{S}^{4} \mathrm{G}$ data set released in Muñoz-Mateos et al. (2015) is also not affected by this typo.

\section{REFERENCE}

Muñoz-Mateos, J. C., Sheth, K., Regan, M., et al. 2015, ApJS, 219, 3 